\title{
Dominance-based Rough Set Approach for Group Decisions
}

\author{
Salem Chakhar ${ }^{\mathrm{a}}$, Alessio Ishizaka ${ }^{\mathrm{a}}$, Ashraf Labib $^{\mathrm{a}}$, Inès Saad ${ }^{\mathrm{b}}$ \\ ${ }^{a}$ Portsmouth Business School and Centre for Operational Research \& Logistics, University of Portsmouth, Portsmouth PO1 3DE, UK. \\ ${ }^{b}$ Amiens Business School and MIS, University of Picardie Jules Verne, 80039 Amiens, France.
}

\begin{abstract}
The objective of this paper is to propose an approach to support group multicriteria classification. The approach is composed of three phases. The first phase exploits the knowledge provided by each decision maker to individually approximate the decision classes using rough approximation. The second phase seeks to combine the outputs of individual approximation phase into a collective decision table by using an appropriate aggregation procedure. The third phase uses the collective decision table in order to infer a set of collective decision rules, which synthesize the judgements and perspectives of the different decision makers and to permit the classification of all decision objects. The proposed approach relies on the Dominance-based Rough Set Approach (DRSA), which is used at two different levels. First, the DRSA is used during the first phase to approximate the input data relative to each decision maker. Second, the DRSA is used during the third phase to approximate the collective decision table and generate the collective decision rules. This paper presents the theoretical foundation of the proposed approach, three case studies using real-world data and a comparative study of recent similar proposals.
\end{abstract}

Keywords: Multicriteria classification, Ordinal classification, Rough approximation, Dominance-based rough set approach, Group decision-making.

\section{Introduction}

The multicriteria classification is a fundamental problem of multicriteria decision-making [36]. The multicriteria classification can be stated as follows: given a set of objects described by a set of criteria (attributes with preferenceordered domains), assign these objects to some pre-defined decision classes or categories, such that each object is assigned to exactly one class. In comparison to other classification methods and techniques (for instance in statistical, data mining, pattern recognition and machine learning), the multicriteria classification has two main characteristics [21][62]: (i) the decision classes are defined in an ordinal way; and (ii) the decision objects are evaluated over a set of criteria meaning that the decision model should have some form of monotonic relationship with respect to the criteria. Due to the first point, the multicriteria classification is often referred to ordinal classification or sorting in the literature. In the rest of the paper, the three terms will be used interchangeably.

A large number of real-world case studies of multicriteria classification problems in different domains are reported in the literature (e.g., [40][70]). In practice, these decision problems often imply several decision makers having conflicting objectives and judgements. However, most multicriteria classification methods assume a single decision maker or a homogenous group of decision makers that acts as a single decision maker. Fortunately, several new methods and decision support systems for group multicriteria classification problems are now available in the literature, including [10][15][17][24][35][37][38][42][47][48]. However, most of existing proposals fail to resolve several group decision making problems shortcomings. Indeed, most of them use either input or output based aggregation strategy. These extreme strategies have some problems as discussed in [14]. In addition, a large number of previous proposals assume that all decision makers have the same "power" and when "weights" are used, they often reflect the hierarchal levels of the decision makers. Another important issue that has not been correctly addressed in the existing works concerns the aggregation rules used to combine individual data and knowledge. In fact, the most used solutions to deal with this issue are either to combine these information informally based

Email addresses: salem.chakhar@port.ac.uk (Salem Chakhar), alessio.ishizaka@port.ac.uk (Alessio Ishizaka), ashraf.labib@port.ac.uk (Ashraf Labib), ines.saad@u-picardie.fr (Inès Saad) 
on discussion between the decision makers or, at best, using a weighted-sum as an aggregation rule that may lead to compensation problems. An additional problem concerns the definition of preference parameters as required by most of multicriteria methods. This is a very difficult exercise in practice which requires an important cognitive effort to the decision makers. Additionally, the elicitation of parameters is more complicated in a group decision context since involved decision makers may not agree on the values of the parameters. Finally, there is a lack of efficient tools to support sensitivity analysis in group decision.

The objective of this paper is to propose an approach to support group decision in multicriteria classification. The proposed approach contains three phases: (i) individual approximation, (ii) aggregation, and (iii) inference of collective decision rules. The individual approximation phase exploits the knowledge provided by each decision maker to approximate individually the collection of upward and downward unions of decision classes using the dominating and dominated sets. The aggregation phase seeks to combine the outputs of individual approximation phase into a collective decision table by using an appropriate aggregation procedure. The inference phase uses the collective decision table in order to infer a set of IF-THEN collective decision rules in order to synthesize the judgements and perspectives of the different decision makers and to permit the classification of all decision objects. The proposed approach relies on the Dominance-based Rough Set Approach (DRSA) [26][27][56], which is used at two different levels. First, the DRSA is used during the first phase to approximate the input data relative to each decision maker. Second, the DRSA is used during the third phase to approximate the collective decision table and generate the collective decision rules. The approach is illustrated in detail through one main case study relative to credit worthiness of 28 European countries and to two other case studies related to heat islands exposition in the Québec Metropolitan Community in Canada and the management of post-accident nuclear risk in the Southern France region. The paper also includes a detailed discussion and justification of the different concepts used in the aggregation procedure.

The approach proposed in this paper has several distinguishing characteristics. First, it adopts a mixed inputoutput aggregation strategy to combine the judgments and perspectives of involved decision makers. The mixed strategy allows taking advantages of both input and output aggregation strategies as explained in [14]. Second, the "power" of the decision makers that measure the contribution of each one in the assignment of decision objects to decision classes is computed based on the input data. As stressed in [14], the use of input data to deduce the "power" of the decision makers seems generally to be more objective than the other weighting techniques. Third, the assignment of decision objects to decision classes is based on the use of the majority principle and veto effect. These notions are implemented through the concepts of concordance and discordance, which originated from Social Choice theory and are now well established in multicriteria analysis. Fourth, it uses a subset of data to extract and generalize the preferences of the decision makers. This idea has been proven to be very useful in practice (see, e.g., [33][49]). Fifth, the approach is enhanced with several tools in order to permit the comparison of the final results obtained using different input parameters. This will substantially improve the effectiveness of the decision making process and the successful implementation of the final solution (see, e.g., [35]).

The paper is organized as follows. Section 2 discusses related work. Section 3 presents the background. Section 4 introduces the approach. Section 5 details the aggregation procedure. Section 6 illustrates the approach through a case study. Section 7 applies the approach to two additional cases studies. Section 8 provides rules to compare different final results. Section 9 concludes the paper.

\section{Related work}

In this section, we first comment on the main characteristics of the DRSA (Section 2.1). Then, we discuss the different extensions of DRSA to group decision making (Section 2.2).

\subsection{The characteristics of the DRSA}

The DRSA is a well-known multicriteria classification method that has been proposed by [26][27][56] to overcome the shortcomings of the conventional Rough Sets Theory (RST) [51] in multicriteria classification. The basic idea of DRSA is to replace the indiscernibility relation used in the classical RST with the dominance relation, which is more appropriate for multicriteria decision-making. The DRSA has been successfully used in different realworld decision problems including risk assessment [13], weed species identification [33], pollution risk assessment of drinking water [43], nuclear risk assessment [12], knowledge management [54], service improvement [46], 
bankruptcy risk evaluation [28], customer satisfaction [30], sustainable planning strategies for farms [50], location of undesirable facilities [1], metabolomics [4] and product mix [25].

The DRSA has some powerful characteristics that makes it attractive in real-world decision problems:

- there is no need of preference parameters, which reduces the cognitive effort required from the decision makers;

- it produces IF-THEN decision rules, which are more simple and easily understandable by the decision makers, especially those with no background in multicriteria decision making [6];

- it is able to deal with incomplete/missing attribute values (see [7][56]);

- it is able to detect and deal with inconsistency problems (see [19][58]);

- it uses a subset of data (as a learning set) to extract and generalize the preferences of the decision makers, which minimizes the cognitive effort required from them.

The last point, concerning the use of a learning set as input, is adapted in several multicriteria classification methods, including [8][20][23]. However, the main addition of DRSA compared to other multicriteria classification methods, that are based on the use of a learning set as input (such as [2][3]), is the simplicity and the easily understandable IF-THEN decision rules provided as output, while other methods have no such straightforward interpretation [6].

There are several extensions to DRSA in the literature. The DRSA has been extended to deal with multicriteria choice and ranking problems in [27][41]. The Variable Consistency Dominance-based Rough Set Approach (VCDRSA) [32] is a variant of DRSA that enables the relaxation of the conditions for assignments of objects to the lower approximations by accepting a limited proportion of negative examples, which is particularly useful for large decision tables. The Stochastic DRSA, which allows inconsistencies to some degree, has been introduced in [18]. In [5], the authors studied the classification of objects using decision rules in situation where it is covered by no rule, exactly one rule and several rules. A parameterized DRSA to deal with interval-valued information systems is presented in [67] and an incremental approach for maintaining approximations of DRSA when attribute values vary over time is proposed in [45]. The authors in [61] provide a formal and unified framework to define and compute inter-class reducts, intra-class reducts and constructs across the conventional RSA and the DRSA. Recently, the DRSA is combined with the robust ordinal regression paradigm in order to obtain robust conclusions [39][57]. There are also several extensions of DRSA for group decision making, which will be briefly discussed in Section 2.2.

The DRSA is useful for dealing with ordered data or for ordinal classification problems with monotonicity constraints. However, in some real-world decision problems, we may not know a-priori if such an ordering exists. In this case, it may be necessary to use initially more traditional inference mechanisms in order to learn from the data before applying DRSA. This topic is further discussed in [24][56]. There is also another solution which consists in applying a transformation allowing to deal with non ordinal data in DRSA as in [6][63].

\subsection{The DRSA extensions to group decision making}

There are several extensions of DRSA to support group multicriteria classification. In two previous research papers [13][14], we discussed and compared several proposals for group decision making with respect to several criteria, including aggregation strategy (at the input level, at the output level, or mixed input-output), aggregation rule (statistical, functional, rule-based techniques or by discussion), preference parameters requirement (criteria weights, parameters, etc.), preference parameters elicitation technique (direct, indirect, mixed), stakeholder weighting (by a mediator or an external person, based on the hierarchical levels of decision makers, using a dedicated method, based on input data) and support of robust assignment. In this paper, we discus some extensions of DRSA to support group decision-making.

The authors in [53] propose a three phase DRSA-based methodology for the identification of crucial knowledge. Using this methodology allows to take into account the preferences of decision makers that can be different or even contradictory while exploiting and managing their multiple points of view to evaluate knowledge, without using a quantitative measuring approach. The methodology proposed in [53] requires, when inconsistency situations are 
identified, that the analyst conducts an in-depth discussion with the different decision makers in order to solve the conflicts. This is a time-consuming and difficult task. For this purpose, the authors in [9] enhance [53]'s methodology by proposing an argumentative multi-agent model based on a mediator agent in order to automate the resolution of conflicts between decision makers.

Another extension of DRSA to support multiple decision makers is reported in [29]. In this paper, the authors extend the lower and upper approximations and boundary concepts of DRSA. More specifically, they introduce the concepts of downward and upward multi-union and mega-union. These new concepts are then used to define lower and upper approximations for unions of classes. In [65], the authors present a rough set approach to group decisions-making very similar to the work of [29]. To deal with the decision of multiple decision makers they extend DRSA by introducing specific concepts related to dominance with respect to minimal profiles of evaluations given by multiple decision makers. In particular, the authors characterize the conditions for a consensus attainable by multiple decision makers considered as a whole. Such a perspective permits to handle interactions between the decision makers.

A system that aggregates case-based linguistic decision rules using a hybrid of the DRSA and the DempsterShafer theory of evidence is proposed for multicriteria sorting implying multiple participant in [15]. The methodology proposed in [15] can be organized in two phases. First, DRSA is employed to infer linguistic decision rules that estimate the preferences of a few participants by means of their evaluations of representative case sets. Next, Dempster-Shafer Theory is applied to aggregate the decision rules triggered by all participants' evaluations of an alternative, thereby generating an overall decision recommendation for the alternative. One main shortcomings of [15]'s proposal is its complexity in the sense that it requires a solid technical and mathematical background in rough approximation and Dempster-Shafer theory of evidence.

In [13], the authors propose a two-phase methodology to support groups in multicriteria classification problems. The major addition of [13] is an aggregation procedure implementing the majority principle and veto effect - in terms of aggregation rules - thus it allows obtaining consensual decisions. Furthermore, the contribution of each decision maker to the collective decision is objectively measured by the quality of individual classification conducted by this decision maker during the first phase. The proposal of [13] has been adopted in [54] to the case of binary decision classes. In [11], the authors refine the aggregation procedure proposed in [13] by replacing the aggregation rules by more simple and more advanced ones requiring only one parameter (namely, credibility threshold) instead of two parameters (namely, majority and veto thresholds) in [13].

The paper [69] proposes a two-stage dynamic group decision making method for aggregating ordinal preferences. The method permits to avoid two problems of group decision making in relation to ordinal preferences - namely, difficulty of managing conflicting opinions and neglecting the power relationships between the preferences provided by the decision makers. Although the proposal of [69] is not primary concerned by the extension of the DRSA to group decision making, it is fruitful to mention that the authors are inspired by the principle of the DRSA in order to define new support function associated with the Power Average operator used to combine the collective preference. As argued by the authors, the use of DRSA's idea permits to better cope with ordinal preferences given by the decision makers. Additionally and as the support function is defined based on DRSA, the aggregation method can be easily extended to other quasi-ordinal preference relations. Furthermore, the non-parameterized definition of the support function (due to DRSA), provides a feasible method to determine the weights of each decision maker.

In [60], the authors present a generalization of the classical DRSA into multi-decision preference dominancebased rough set model supporting a set of condition criteria and a set of decision classes. The basic idea of [60] is to define two dominance relations, one on the condition criteria set and the other on the decision classes set, which are then used to define the lower and upper approximations. The authors propose four versions of their decision model, which are then applied to solve a multi-agent conflict analysis decision problem and improve the Pawlak conflict analysis model [52].

Finally, it is important to mention that the approach proposed in this paper enhances the one proposed in [13]. First, this approach uses more advanced weighing system leading to more realistic and precise weights, as explained in Sections 5.1. Second, it replaces the two assignment rules in [13] by a single assignment rule, which reduces the number of required parameters and which is more appropriate for sensitivity analysis as illustrated in Section 6.4. Third, the paper provides a detailed justification of the concepts used including the definition of decision makers' weights (Section 5.1), the definition of the concordance power (Section 5.2), the definition of the discordance power (Section 5.3) and the definition of assignment intervals (Section 5.5). Fourth, the paper includes new case studies that are relative to credit worthiness of 28 European countries (Section 6), heat islands exposition in the 
Québec Metropolitan Community in Canada (Section 7.1) and the management of post-accident nuclear risk in the Southern France region (Section 7.2). Lastly, a collection of well-known statistics (namely Kendall's $\tau$, Spearman's $\rho$, Cohen's $\kappa$, Kendall's $W$ and Fless's $\kappa$ ) is used to compare in a formal way the final classifications obtained using different input parameters (Section 8).

\section{Dominance-based Rough Set Approach}

This section presents a brief introduction to the DRSA. More details are available in [26][27][56].

\subsection{Basic concepts and hypothesis}

Information regarding the decision objects is often structured in a 4-tuple information table $\mathbf{S}=\langle U, Q, V, f\rangle$, where $U$ is a non-empty finite set of objects and $Q$ is a non-empty finite set of attributes such that $q: U \rightarrow V_{q}$ for every $q \in Q . V_{q}$ is the domain of attribute $q . V=\bigcap_{q \in Q} V_{q}$, and $f: U \times Q \rightarrow V$ is the information function defined such that $f(x, q) \in V_{q}$ for each attribute $q$ and object $x \in U$. The set $Q$ is often divided into a sub-set $C \neq \emptyset$ of condition criteria and a sub-set $D \neq \emptyset$ of decision classes such that $C \cup D=Q$ and $C \cap D=\emptyset$. In this case, $\mathbf{S}$ is called a decision table.

The domain of condition criteria are supposed to be ordered according to a decreasing or increasing preference. Such attributes are called criteria. The proponents of DRSA assume that the preference is increasing with a value of $f(\cdot, q)$ for every $q \in C$. We also assume that the set of decision classes $D=\{d\}$ is a singleton. The unique decision attribute $d$ makes a partition of $U$ into a finite number of preference-ordered decision classes $\mathbf{C l}=\left\{C l_{t}, t \in T\right\}$, $T=\{0, \cdots, n\}$, such that each $x \in U$ belongs to one and only one class.

\subsection{Approximations}

In DRSA the represented knowledge is a collection of upward union $C l_{t}^{\geq}$and downward union $C l_{t}^{\leq}$of classes defined as follows:

$$
C l_{t}^{\geq}=\bigcup_{s \geq t} C l_{s}, C l_{t}^{\leq}=\bigcup_{s \leq t} C l_{s} .
$$

The assertion " $x \in C l_{t}^{\geq}$" means that " $x$ belongs to at least class $C l_{t}$ " while assertion " $x \in C l_{t}^{\leq}$" means that " $x$ belongs to at most class $C l_{t}$ ". As underlined earlier, the basic idea of DRSA is to replace the indiscernibility relation used in the conventional RST with the dominance relation. Let $P \subseteq C$ be a subset of condition criteria. The dominance relation $\Delta_{P}$ associated with $P$ is defined for each pair of objects $x$ and $y$ as follows:

$$
x \Delta_{P} y \Leftrightarrow f(x, q) \succeq f(y, q), \forall q \in P .
$$

In the definition above, the symbol " $\succeq$ " should be replaced with “ $\preceq$ ” for criteria which are ordered according to decreasing preferences. To each object $x \in U$, we associate two sets: (i) the P-dominating set $\Delta_{P}^{+}(x)=\{y \in$ $\left.U: y \Delta_{P} x\right\}$ containing the objects that dominate $x$, and (ii) the $P$-dominated set $\Delta_{P}^{-}(x)=\left\{y \in U: x \Delta_{P} y\right\}$ containing the objects dominated by $x$.

Then, the $P$-lower and $P$-upper approximations of $C l_{t}^{\geq}$with respect to $P$ are defined as follows:

- $\underline{P}\left(C l_{t}^{\geq}\right)=\left\{x \in U: \Delta_{P}^{+}(x) \subseteq C l_{t}^{\geq}\right\}$,

- $\bar{P}\left(C l_{t}^{\geq}\right)=\left\{x \in U: \Delta_{P}^{-}(x) \cap C l_{t}^{\geq} \neq \emptyset\right\}$.

Analogously, the $P$-lower and $P$-upper approximations of $C l_{t}^{\leq}$with respect to $P$ are defined as follows:

- $\underline{P}\left(C l_{t}^{\leq}\right)=\left\{x \in U: \Delta_{P}^{-}(x) \subseteq C l_{t}^{\leq}\right\}$,

- $\bar{P}\left(C l_{t}^{\leq}\right)=\left\{x \in U: \Delta_{P}^{+}(x) \cap C l_{t}^{\leq} \neq \emptyset\right\}$.

The $P$-boundaries of $C l_{t}^{\geq}$and $C l_{t}^{\leq}$are defined as follows:

- $B n_{P}\left(C l_{t}^{\geq}\right)=\bar{P}\left(C l_{t}^{\geq}\right)-\underline{P}\left(C l_{t}^{\geq}\right)$,

- $B n_{P}\left(C l_{t}^{\leq}\right)=\bar{P}\left(C l_{t}^{\leq}\right)-\underline{P}\left(C l_{t}^{\leq}\right)$. 


\subsection{Quality of approximation}

The quality of approximation of a partition $\mathbf{C l}$ by means of a set of criteria $P$ is measured by the following ratio:

$$
\gamma(\mathbf{C l})=\frac{\left|U-\left(\left(\bigcup_{t \in T} B n_{P}\left(C l_{t}^{\geq}\right)\right) \bigcup\left(\bigcup_{t \in T} B n_{P}\left(C l_{t}^{\leq}\right)\right)\right)\right|}{|U|} .
$$

It expresses the ratio of all $P$-correctly classified objects to all objects in the system.

\subsection{Accuracy of classes approximation}

The accuracy of the rough-set representation of classes is the ratio of the number of objects which can positively (i.e., in the lower approximation) be placed into the number of objects that can possibly (i.e., in the upper approximation) be placed in. Mathematically,

$$
\alpha\left(C l_{t}^{\diamond}\right)=\frac{P}{\bar{P}\left(C l_{t}^{\diamond}\right)}
$$

where $\diamond \in\{\geq, \leq\}$. It is easy to see that $0 \leq \alpha\left(C l_{t}^{\diamond}\right) \leq 1, \forall t$. This holds because, by definition, we have: $\underline{P}\left(C l_{t}^{\diamond}\right) \subseteq \bar{P}\left(C l_{t}^{\diamond}\right), \forall t$. Clearly, when the upper and lower approximations are equal (i.e., boundary region empty), then $\alpha\left(C l_{t}^{\diamond}\right)=1$, and the approximation is perfect. At the other extreme, whenever the lower approximation is empty, the accuracy is zero, i.e., $\alpha\left(C l_{t}^{\diamond}\right)=0$.

\subsection{Decision rules}

The decision attribute induces a partition of $U$ in a way that is independent of the condition criteria. Hence, a decision table may be seen as a set of decision rules of the form if [condition] then [consequence], where the condition part specifies the values assumed by one or more condition criteria and the decision part specifies an assignment to one or more decision classes. Three types of decision rules may be considered: (i) certain rules generated from lower approximations of unions of classes, (ii) possible rules generated from upper approximations of unions of classes and (iii) approximate rules generated from boundary regions.

An object $x \in U$ supports a decision rule if its description matches both the condition and the decision parts of this rule. A decision rule covers object $x$ if the description of $x$ matches at least the condition part of the rule. Each decision rule is characterized by its strength, which is defined as the number of objects supporting this rule. If the consequence is univocal (i.e., contains only one decision), the rule is exact, otherwise it is approximate.

\section{Dominance rough set approach for group multicriteria classification}

The objective of this section is to introduce the Dominance Rough Set Approach for Group (DRSAfG) multicriteria classification. First, we will briefly present the aggregation strategy adopted by DRSAfG. Then, we describe the different phases of DRASfG. Next, we discuss some practical issues. Lastly, we comment on the definition of decision classes.

\subsection{Aggregation strategy}

As underlined in [14], we may distinguish two main strategies that may be used to combine judgements and perspectives of decision makers in group decision making: either at the input or at the output levels. These two strategies still apply to group multicriteria classification. The input oriented aggregation strategy seems to be technically and practically easier to implement and to use. However, it assumes that decision makers collaborate effectively and that there is not much conflict regarding the input data. The output oriented strategy seems to be more flexible since the involved decision makers can work independently. Hence, it is more suitable for decision makers that are geographically distributed and/or those that have time constraints. However, the output aggregation strategy is technically more complex.

The DRSAfG is structured according to a mixed input-output strategy that allows avoidance or reduction of the respective shortcomings of the input and output strategies. The mixed aggregation strategy proceeds as follows [14]: (i) each decision maker generates his/her own output (approximations of decision classes); (ii) aggregating coherently the individual outputs into a collective input (collective decision table); and (iii) use the collective input in order to generate a collective output (collective decision rules). A detailed description of the three aggregation strategies is given in [14]. 


\subsection{The general structure of DRSAfG}

The DRSAfG is composed of three phases: (i) individual approximation, (ii) aggregation, and (iii) inference of collective decision rules. These phases should be mapped directly into the three phases of the mixed input-output aggregation strategy described previously. Let $H=\{1, \cdots, i, \cdots, h\}$ with $h \geq 2$ be a finite decision makers set and $\mathbf{I}=\langle U, Q, V, f\rangle$ be a common information table for all decision makers. Let $E_{1}, \cdots, E_{i}, \cdots, E_{h}$ be $h$ decision attributes defined in the same domain and associated with decision makers in $H$. Let $\diamond \in\{\geq, \leq\}$.

\subsubsection{Individual approximation}

In this phase, each decision maker uses the common information table $\mathbf{I}$ to construct her/his own decision table $\mathbf{S}_{i}=\left\langle U, C \cup\left\{E_{i}\right\}, V, f_{i}\right\rangle$ where $E_{i}$ and $f_{i}$ are respectively the decision class and the information function associated with the $i$ th decision maker. We suppose that each decision maker $i \in H$ has a preference order for $U$ represented by a finite set of preference-ordered classes $\mathbf{C l}_{i}=\left\{C l_{t, i}, t \in T_{i}\right\}, T_{i}=\{0, \cdots, n\}$, such that $\bigcup_{t=1}^{n} C l_{t, i}=U$, $C l_{t, i} \cap C l_{r, i}=\emptyset, \forall r, t \in T_{i}, r \neq t$. The $n$ is the number of decision classes, which is assumed to be the same for all decision makers. Some solutions to dealt with different number of decision classes are briefly discussed in Section 4.4. Each decision table $\mathbf{S}_{i}$ can then be represented as a collection of upward and downward unions of decision classes and approximated in the same way as with the DRSA. At the end of this phase, the approximation of each individual decision table $\mathbf{S}_{i}$ is characterized, among others, by: (i) the $P$-lower approximation and the $P$-boundary of $C l_{t, i}^{\diamond}, \forall t \in T_{i}$, (ii) the quality of classification $\gamma_{i}$ defined in similar way to Equation (1), and (iii) the accuracy of classes approximations $\alpha\left(C l_{t}^{\diamond}\right), \forall t$, defined in similar way to Equation (2).

\subsubsection{Aggregation}

The objective of this phase is to construct a collective decision table $\mathbf{S}=\langle U, C \cup D, V, g\rangle$ where $D=\{E\}$, $E$ is a collective decision attribute and $g$ is a collective information function defined for each $x \in U$ such that $g(x, q)=f(x, q), \forall q \in C$. Hence, to define $\mathbf{S}$ it suffices to specify the values of $g(x, E)$ for all $x \in U$. For this purpose, we designed an aggregation procedure that will be detailed in Section 5. The basic idea of this procedure is to use the outputs of individual approximation phase to assign to each object $x \in U$ an assignment interval $I(x)=[l(x), u(x)]$ where $l(x)$ and $u(x)$ are respectively the lower and upper classes to which object $x$ can be assigned, and then some simple rules are used to reduce the assignment interval $I(x)$ into a single element representing the value of $g(x, E)$. The collective decision attribute $E$ induces a partition of $U$ into a set of decision classes $\mathbf{C l}=\left\{C l_{t}, t \in T\right\}, T=\{0, \cdots, n\}$ such that each $x \in U$ belongs to one and only one class $C l_{t} \in \mathbf{C l}$.

\subsubsection{Inference of collective decision rules}

The collective decision table $\mathbf{S}$ constructed in the previous phase can be approximated using the same principle given in Section 3, i.e., by applying conventional DRSA. On the basis of the approximations obtained, it is possible to induce a generalized description of the preferential information contained in the decision table, in terms of collective decision rules of the form if [condition] then [consequence]. The obtained rules are then used to classify all decision objects. The most popular rule induction algorithm for DRSA is DOMLEM (DOMinance-based Learning from Examples Module) [31], which generates minimal set of rules. The same algorithm is used here.

\subsection{Practical aspects}

The approach is structured as an iterative decision-making process. Specifically, at the end of the third phase, a set of collective decision rules are generated. If the involved decision makers agree on these rules, then the decision-making process ends. Otherwise, the process can then be restarted by considering new input data.

The aggregation procedure requires the definition of two parameters. The first one is called credibility threshold. The latter represents the minimum value for the credibility index for assigning an object to a given class or union of classes. The second parameter is the interval reduction rule. This parameter will be used to reduce the assignment interval into a single value corresponding to the value of the collective decision attribute. These parameters should be fixed by the mediator but the decision makers involved should agree on them. In practice, these parameters will be of great importance since the mediator can use them in order to test different possible values and to select the ones that fit better to the decision situation. 


\subsection{Definition of decision classes}

In the description of DRSAfG, we assumed that the decision makers use the same set of decision classes. However, in practice, the number of decision classes may differ from one decision maker to another. There are (at least) four possible solutions to this issue:

- a first intuitive solution consists of the fact that the decision makers should first discuss and agree on a common set of decision classes before starting the assignment process;

- a second simple solution consists of grouping some decision classes;

- a third simple solution that consists of adding fictitious classes (without assigning objects to them);

- a fourth, more established, solution consists of defining a set of common decision classes as the Cartesian Product of individual decision classes.

The implementation of the first solution is straightforward and does not need any modification in the proposed methodology. The second solution is also simple to implement, but it may lead to preferential information loss. For the third solution, we need only to re-arrange the assignments of the decision objects according to the new definition of the decision classes. Let for instance assume that the decision problem involves two decision makers with two sets of decision classes: $C l_{a} \prec C l_{b} \prec C l_{c}$ and $C l_{x} \prec C l_{y}$. According to the third solution, we need to add a new decision class, say $C l_{\text {new }}$, to the second set of decision classes, which leads to $C l_{x} \prec C l_{\text {new }} \prec C l_{y}$. Then, we can define a new common set of decision classes $C l_{1} \prec C l_{2} \prec C l_{3}$ with $C l_{1}=C l_{a}=C l_{x}, C l_{2}=C l_{b}=C l_{\text {new }}$ and $C l_{1}=C l_{c}=C l_{y}$. The last solution is more complex to implement. The main issue in this case is the ordering of the newly created decision classes. For instance, with the same data as above, the Cartesian Product will lead to six decision classes $C l_{1}=\left(C l_{a}, C l_{x}\right), C l_{2}=\left(C l_{a}, C l_{y}\right), C l_{3}=\left(C l_{b}, C l_{x}\right), C l_{4}=\left(C l_{b}, C l_{y}\right), C l_{5}=\left(C l_{c}, C l_{x}\right)$ and $C l_{6}=\left(C l_{c}, C l_{y}\right)$. However, the new common decision classes cannot be directly used because some of them are incomparable, for example $C l_{2}$ and $C l_{3}$ or $C l_{4}$ and $C l_{5}$. A possible solution is to merge the incomparable decision classes. Another possible solution is to use some additional information (such as the number of decision makers, the number of assigned decision objects or the quality of individual classifications) to order the incomparable decision classes. For the purpose of this paper, we assume that the decision makers use the same set of decision classes. We note, however, that the above cited solutions will be studied in a more formal way in our future work.

\section{Aggregation procedure}

The objective of this section is to detail the aggregation procedure that is used in the second phase in order to construct the collective decision table $\mathbf{S}=\langle U, C \cup D, V, g\rangle$. As underlined in Section 4, the construction of $\mathbf{S}$ requires simply the specification of the values of the collective information function $g$ in respect to the collective decision attribute $E$. First, recall that the collective decision attribute $E$ induces a partition of $U$ into a set of decision classes $\mathbf{C l}=\left\{C l_{t}, t \in T\right\}, T=\{0, \cdots, n\}$ such that each $x \in U$ belongs to one and only one class $C l_{t} \in \mathbf{C l}$. Let $P \subseteq C$ be a subset of condition criteria and let $\diamond \in\{\leq, \geq\}$.

\subsection{Step 1. Weighting the contribution of decision makers}

The definition of the weights of decision makers is a crucial step in group decision making [69]. The authors in [14] enumerated several techniques to specify the weights in group decision making: (i) weights are defined explicitly by a mediator or an external independent person as in [44]; (ii) weights are defined based on the hierarchical levels of involved decision makers; (iii) weights are defined explicitly using a given method as in [34][68]; and (iv) weights are inferred from input data using some form of regression as in [20]. Each of these techniques have some advantages and disadvantages and the selection of the technique to use is not obvious. The authors argue that the most important characteristic of weights definition method is the objectiveness of these weights. In this respect, it is advocated that the question is not how to use weights, but rather how to objectively quantify them [16]. Another important characteristic of weights definition methods is the ability of these methods to objectively measure the expertise of the decision makers or experts, as discussed in [34][55][64]. Indeed and as pointed out by [34], more experienced decision makers will generally provide more consistent decisions. This is confirmed in different real-world applications in which the authors were involved, as e.g., [49][53]. 
In the DRSAfG, the assignment of objects to decision classes requires the definition of an appropriate weighting system that reflects in the most objective way the role of each decision maker in the collective decision. In this paper, the contribution of each decision maker to the collective decision is measured by the quality of input data provided by the decision maker. More formally, we propose the following formula:

$$
\pi_{k}\left(C l_{t}^{\diamond}\right)=\frac{\gamma_{k} \alpha_{k}\left(C l_{t}^{\diamond}\right)}{\sum_{r \in H_{t}^{\diamond}} \gamma_{r} \alpha_{r}\left(C l_{t}^{\diamond}\right)+\sum_{r \notin H_{t}^{\diamond}} \gamma_{r}}, \quad(\forall k \in H)(\forall t \in T)
$$

where $\diamond \in\{\geq, \leq\}, H_{t}^{\diamond}=\left\{j: j \in H \wedge \alpha_{j}\left(C l_{t}^{\diamond}\right)>0\right\}, \gamma_{k}$ is the quality of approximation of decision maker $k$ and $\alpha_{k}\left(C l_{t}^{\diamond}\right)$ is the accuracy of approximation of class $C l_{t}^{\diamond}$ by decision maker $k$. This form of weights definition combines both the quality of classification and the accuracy of the rough-set representation of classes. It enhances the weighting system used in [11][13], which is based on the quality of approximation only. In fact, the accuracy of the approximation of an individual class, say $C l_{t}$, obtained by a given decision maker, say $k$, may be equal to 0 which means that decision maker does not support any assignment of objects to class $C l_{t}$. This fact is well supported by the proposed weighting system. Using just the quality of approximation as weights as in [11][13], it does not allow to take into account this fact (since the quality of approximation characterizes the whole classification rather than the approximations of individual classes). In the new form of weights definition, an accuracy of the approximation of class $C l_{t}$ equal to 0 leads to a weight of zero meaning that the decision maker does not support the assignment of objects to the class $C l_{t}$. The main argument of using the accuracy of approximation is that a given decision maker may be able to define more precisely some classes than other ones. For instance, in a problem of assigning students to three decision classes designed as Bad, Medium, Good, it is expected that one can identify perfectly good students but it has more difficulty to identify bad or medium students.

It is very important to mention that the weights of decision makers as used in this paper should be interpreted as used in non-compensatory multicriteria methods, especially those based on outranking relations, where the weights play the same role as a number of votes in a voting procedure. This contrast with weights definition in compensatory multicriteria methods (the weighted-sum like methods) where weights are actually scaling constant (this means that if you change the unit in which is expressed a criterion, the weights change as well).

A final issue about weights definition concerns the controversial situation when a given decision maker provides fully consistent but controversial choices, where 'controversial' means different than the majority of decision makers. In such a situation the decision maker is more 'powerful' than the others, who are less consistent but also less controversial. This situation is realistic and can happen in real-world decision problems. However, the use of the majority/veto rule reduces largely the effects of the controversial assignments on the final outputs. In addition, the weights are specific to each class union. This means that the effects of a controversial assignment of a given individual class will concern only this class. Furthermore, the other decision makers still have the possibility to disagree on the final outputs and to end the decision process as briefly discussed in Section 4.3.

\subsection{Step 2. Computing of the concordance power}

For each $x \in U$ and $C l_{t} \in \mathbf{C l}$, we define the concordance set as $L\left(x, C l_{t}^{\diamond}\right)=\left\{i: i \in H \wedge x \in \underline{P}\left(C l_{t, i}^{\diamond}\right)\right\}$. This set represents the decision makers for which object $x$ belongs to the lower approximation of $C l_{t}^{\diamond}$. The concordance power for the assignment of $x$ to $C l_{t}^{\diamond}$ is then computed as follows:

$$
S\left(x, C l_{t}^{\diamond}\right)=\sum_{k=1}^{k=n} S_{k}\left(x, C l_{t}^{\diamond}\right)
$$

where

$$
S_{k}\left(x, C l_{t}^{\diamond}\right)= \begin{cases}\pi_{k}\left(C l_{t}^{\diamond}\right), & \text { if } k \in L\left(x, C l_{t}^{\diamond}\right), \\ 0, & \text { otherwise. }\end{cases}
$$

The number $S\left(x, C l_{t}^{\diamond}\right)$ measures the power of the coalition of decision makers that assign $x$ to the lower approximation of $C l_{t}^{\diamond}$. The concordance power verifies some properties which are given in Appendix A.1. The concordance power is defined on the basis of lower approximation of classes, which contains objects that are assigned with certainty to a given class. In this sense, it can be seen as an argument that supports the assignment of objects to classes.

Let us now justify the definition of the concordance power as a sum: 
- when all the decision makers support the assignment decision, i.e. $L\left(x, C l_{t}^{\diamond}\right)=H$, the concordance power will be equal to 1 (the maximum value). This will automatically inhibit the veto effect of the discordance power (as explained in Section 5.3) and leads to a full support for the assignment of the decision object $x$ to decision class $C l_{C} l_{t}^{\diamond}$;

- when no decision maker supports the assignment decision, i.e. $L\left(x, C l_{t}^{\diamond}\right)=\emptyset$, the concordance power will be equal to 0 (the minimum value). In this case, the assignment of decision object $x$ to a given class $C l_{t}$ will be rejected since none of the decision makers supports this assignment;

- when only a subset of decision makers support the assignment decision, i.e. $L\left(x, C l_{t}^{\diamond}\right) \neq H$ and $L\left(x, C l_{t}^{\diamond}\right) \neq$ $\emptyset$, the assignment is confirmed only and only if: (i) a majority of decision makers supports this assignment; and (ii) there is not a high opposition to this assignment from the other (minority) decision makers. These two conditions hold if the credibility index (see Section 5.4) for assigning an object $x$ to a given class $C l_{t}$ is greater or equal to 0.5 .

We note that other ways to define the global concordance power may be used. For instance, the global concordance power may be defined as the product of partial concordance powers. However, this definition is not suitable since the global concordance power will be equal to 0 even if there is only one decision maker that does not support the assignment decision. The use of a constrained product of partial concordance powers (to avoid the multiplication by a zero) could be also used. However, this technique is not attractive in our context since the values obtained will still tend towards zero (but strictly positive) because all the weights are in the range of $] 0,1]$.

Finally, we should mention that the definition of the concordance power in this paper is inspired by the definition of the concept of concordance in the ELECTRE methods family [23]. Indeed, in both cases, the global concordance power is defined as the sum of the partial concordance powers. The main difference is that the global concordance power as defined in this paper measures the power of the coalition of decision makers that are in favor of the decision concerning the assignment of a decision object $x$ to a given class $C l_{t}$ while in ELECTRE methods, the concordance measures the coalition of criteria that are in favor of the outranking relation between two decision objects (or an object and a profile limit in the case of the ELECTRE TRI method).

\subsection{Step 3. Computing of the discordance power}

For each $x \in U$ and $C l_{t} \in \mathbf{C l}$, we define the discordance set $B\left(x, C l_{t}^{\diamond}\right)=\left\{i: i \in H \wedge x \in B n_{P}\left(C l_{t, i}^{\diamond}\right)\right\}$. This set represents the decision makers for which object $x$ belongs to the boundary of $C l_{t}^{\diamond}$. Then, the discordance power for the assignment of $x$ to the boundary of $C l_{t}^{\diamond}$ is computed as follows:

$$
Z\left(x, C l_{t}^{\diamond}\right)=\prod_{k=1}^{k=n} Z_{k}\left(x, C l_{t}^{\diamond}\right)
$$

where

$$
Z_{k}\left(x, C l_{t}^{\diamond}\right)= \begin{cases}\frac{1-\pi_{k}\left(C l_{t}^{\diamond}\right)}{1-S\left(x, C l_{t}^{\diamond}\right)}, & \text { if } \pi_{k}\left(C l_{t}^{\diamond}\right)>S\left(x, C l_{t}^{\diamond}\right) \wedge k \in B\left(x, C l_{t}^{\diamond}\right), \\ 1, & \text { otherwise. }\end{cases}
$$

The number $Z\left(x, C l_{t}^{\diamond}\right)$ measures the power of the coalition of decision makers that assign $x$ to the boundary of $C l_{t}^{\diamond}$. The discordance power verifies some properties which are given in Appendix A.2. The definition of the discordance power is based on the boundaries of classes, which contains objects that can neither be ruled in nor out as members of the class. In this sense, it represents an argument that opposes to the assignment of objects to classes.

We may distinguish two cases in the definition of the discordance power. The first case holds when $\pi_{k}\left(C l_{t}^{\diamond}\right) \leq$ $S\left(x, C l_{t}^{\diamond}\right)$, which leads to $Z_{k}\left(x, C l_{t}^{\diamond}\right)=1$. Hence, there is no veto effect for decision maker $k$ and $Z_{k}\left(x, C l_{t}^{\diamond}\right)$ will have no effect on the definition of the overall discordance power $Z\left(x, C l_{t}^{\diamond}\right)$. The second case holds when $\pi_{k}\left(C l_{t}^{\diamond}\right)>S\left(x, C l_{t}^{\gtrless}\right)$, which leads to $0<Z_{k}\left(x, C l_{t}^{\diamond}\right)<1$. Here, decision maker $k$ will have a veto effect, and $Z_{k}\left(x, C l_{t}^{\diamond}\right)$ will have an effect on the value of the overall discordance power.

Let us now justify the definition of discordance power as a product: 
- when all decision makers show an opposition to the assignment decision, i.e. $B\left(x, C l_{t}^{\diamond}\right)=H$, the discordance power will be equal to 0 . This is because the 'IF' condition in Equation (7) will be true for all decision makers. In this case, Equation (7) leads to $Z_{k}\left(x, C l_{t}^{\diamond}\right)=0$ since $1-\pi_{k}\left(C l_{t}^{\diamond}\right)=0, \forall k \in H$. Hence, the value of the global discordance given by (6) will be equal to 0 . Consequently, the credibility index will be equal to 0 (as explained in Section 5.4) and the assignment is rejected. This is an implicit form of veto effect because all the decision makers are against the assignment;

- when there is no decision maker opposing to the assignment decision, i.e. $B\left(x, C l_{t}^{\diamond}\right)=\emptyset$, the discordance power will be equal to 1. This is because the 'IF' condition in Equation (7) will be false for all decision makers. Hence, the assignment should be accepted since it is supported by all decision makers. Here, there is no veto effect (all the decision makers agree on the assignment);

- when only a subset of decision makers are opposed to the assignment decision, i.e. $B\left(x, C l_{t}^{\diamond}\right) \neq H$ and $B\left(x, C l_{t}^{\diamond}\right) \neq \emptyset$, both the concordance and discordance powers will be in the range of $] 0,1[$. In this case, the final decision depends on both concordance and discordance powers. If the global discordance power $Z\left(x, C l_{t}^{\diamond}\right)$ is strong enough, the assignment decision is rejected. This is an explicit form of veto effect. If the global discordance power $Z\left(x, C l_{t}^{\diamond}\right)$ is not strong enough, it will reduce the support level but not as much as required to reject the assignment. This is also an explicit form of veto effect but the veto is not strong enough to lead to the rejection of the assignment decision.

Finally, we should mention that the definition of the discordance power in this paper is inspired by the definition of the concept of discordance in the ELECTRE methods family [23]. Indeed, in both cases, the global discordance power is defined as the product of the partial discordance powers. The main difference is that the global discordance power as defined in this paper measures the power of the coalition of decision makers that are not in favor of the decision concerning the assignment of a decision object $x$ to a given class $C l_{t}$ while in ELECTRE methods, the discordance measures the coalition of the criteria that are not in favor of the outranking relation between two decision objects (or an object and a profile limit in the case of the ELECTRE TRI method).

\subsection{Step 4. Computing of the credibility indices}

Using the concordance and discordance powers, we may compute the credibility indices for assigning $x$ to $C l_{t}^{\diamond}$ as follows:

$$
\sigma\left(x, C l_{t}^{\diamond}\right)=S\left(x, C l_{t}^{\diamond}\right) \cdot Z\left(x, C l_{t}^{\diamond}\right)
$$

The credibility index verifies two properties which are given in Appendix A.3. This formula can be explained as follows. If there is no support for the assignment of $x$ to $C l_{t}^{\diamond}$, i.e., $S\left(x, C l_{t}^{\diamond}\right)=0$, then the credibility index will be $\sigma\left(x, C l_{t}^{\diamond}\right)=0$. In turn, if there is a full support, i.e., $S\left(x, C l_{t}^{\diamond}\right)=1$ (which imposes that $Z\left(x, C l_{\dot{t}}\right)=1$ ), then the credibility index will be $\sigma\left(x, C l_{t}^{\diamond}\right)=1$. Finally, if there is a partial support, i.e., $0<S\left(x, C l_{t}^{\diamond}\right)<1$ (which imposes that $\left.0<Z\left(x, C l_{t}^{\diamond}\right) \leq 1\right)$, then $0<\sigma\left(x, C l_{t}^{\diamond}\right)<1$.

In the last case, we may distinguish between two subcases, according to the verification or not of the condition $\pi_{k}\left(C l_{t}^{\diamond}\right)>S\left(x, C l_{t}^{\diamond}\right)$ (which is used in Equation (7)). The first subcase holds when the condition $\pi_{k}\left(C l_{t}^{\diamond}\right)>$ $S\left(x, C l_{t}^{\diamond}\right)$ is not verified. This leads to $Z\left(x, C l_{t}^{\diamond}\right)=1$ and then $\sigma\left(x, C l_{t}^{\diamond}\right)=S\left(x, C l_{t}^{\diamond}\right)<1$. In this subcase, the credibility index is simply equal to the concordance power; hence the discordance power will have no effect on the value of the credibility index $\sigma\left(x, C l_{t}^{\diamond}\right)$. The second subcase holds when the condition $\pi_{k}\left(C l_{t}^{\diamond}\right)>S\left(x, C l_{t}^{\diamond}\right)$ is verified. This leads to $Z\left(x, C l_{t}^{\diamond}\right)<1$ and consequently $\sigma\left(x, C l_{t}^{\diamond}\right)=S\left(x, C l_{t}^{\diamond}\right) \cdot Z\left(x, C l_{t}^{\diamond}\right)<1$. In this subcase, the credibility index is obtained by decreasing the concordance power $S\left(x, C l_{t}^{\diamond}\right)$ proportionally to the value of the discordance power $Z\left(x, C l_{t}^{\diamond}\right)$.

\subsection{Step 5. Definition of assignment intervals}

The objective of this step is to exploit the credibility indices computed in the previous step in order to assign each decision object $x \in U$ to an assignment interval $I(x)=[l(x), u(x)]$ where $l(x)$ and $u(x)$ with $l(x) \preceq u(x)$ indicate the lower and upper bounds to which decision object $x$ should be assigned. The assignment of a decision object $x \in U$ into decision class $C l_{t}^{\diamond}$ will depend on the value of credibility indices values. The credibility indices range in $[0,1]$ where a value of 1 indicates a full support for assigning $x$ to $C l_{t}^{\diamond}$ and a value of 0 indicates a total disagreement 
with this assignment. A value in the range ] $0,1\left[\right.$ indicates an intermediate support for assigning $x$ to $C l_{t}^{\diamond}$. From a decision making perspective, a credibility index value $\sigma\left(x, C l_{t}^{\geq}\right)$verifying the constraint $\sigma\left(x, C l_{t}^{\geq}\right) \geq 0.5$ ensures that at least $50 \%$ of decision makers (in terms of their powers) support the assignment of decision object $x$ to decision class $C l_{t}^{\diamond}$.

Let $\lambda \in[0.5,1]$ be a credibility threshold. Then, based on the discussion above, we may distinguish two types of constraints for assigning objects to decision classes: (i) $\sigma\left(x, C l_{t}^{\geq}\right) \geq \lambda$ (type ' $\geq$ ' constraint) and (ii) $\sigma\left(x, C l_{t}^{\leq}\right) \geq \lambda$ (type ' $\leq$ ' constraint). The lower $l(x)$ and uppers $u(x)$ bounds of the assignment interval $I(x)$ can be defined based on the two types of constraints. This leads to four possible solutions for defining $l(x)$ and $u(x)$ (see Table 1). An appropriate assignment interval $I(x)=[l(x), u(x)]$ should, however, verify the following conditions: (i) the lower bound $l(x)$ should indicate the minimum class to which a given decision object $x$ is assigned. Consequently, it should be defined based on a constraint of the type ' $\geq$ '; and (ii) the upper bounds $u(x)$ should indicate the maximum class to which a given decision object $x$ is assigned. Consequently, it should be defined based on a constraint of the type of ' $\leq$ '. Hence, Solutions 1, 3 and 4 cannot be used since: ( $a$ ) Solution 1 fails to verify condition (ii), (b) Solution 4 fails to verify condition (i), and (c) Solution 3 fails to verify conditions (i) and (ii). Hence, only Solution 2, which has been used in this paper, is valid since it verifies both conditions.

\begin{tabular}{|l|l|l|}
\multicolumn{4}{c}{ Table 1: Solutions for defining $l(x)$ and $u(x)$} \\
\hline Solutions & \multicolumn{1}{c|}{$l(\mathrm{x})$} & \multicolumn{1}{c|}{$\mathrm{u}(\mathrm{x})$} \\
\hline Solution 1 & Based on constraints of type $\geq$ & Based on constraints of type $\geq$ \\
Solution 2 & Based on constraints of type $\geq$ & Based on constraints of type $\leq$ \\
Solution 3 & Based on constraints of type $\leq$ & Based on constraints of type $\geq$ \\
Solution 4 & Based on constraints of type $\leq$ & Based on constraints of type $\leq$ \\
\hline
\end{tabular}

Based on this discussion, we associate to each object $x$ a collective assignment interval $I(x)=[l(x), u(x)]$ such that:

$$
\begin{aligned}
& l(x)= \begin{cases}\operatorname{argmax}_{C l_{t}} N_{1}(x), & \text { if } N_{1}(x) \neq \emptyset, \\
C l_{0}, & \text { otherwise. }\end{cases} \\
& u(x)= \begin{cases}\operatorname{argmin}_{C l_{t}} N_{2}(x), & \text { if } N_{2}(x) \neq \emptyset, \\
C l_{n}, & \text { otherwise. }\end{cases}
\end{aligned}
$$

with $N_{1}(x)=\left\{C l_{t}: \sigma\left(x, C l_{t}^{\geq}\right) \geq \lambda\right\}$ and $N_{2}(x)=\left\{C l_{t}: \sigma\left(x, C l_{t}^{\leq}\right) \geq \lambda\right\}$. The assignment interval verifies several properties which are given in Appendix A.4. It is easy to see that our definition of the lower $l(x)$ and upper $u(x)$ bounds of the assignment interval $I(x)$ apply even when $N_{1}(x), N_{2}(x)$ or both are empty.

Let us now justify the definition of $l(x)$ and $u(x)$ as in Equations (9) and (10). There are four possible cases to specify the values of $l(x)$ and $u(x)$. These solutions are given in Table 2 and represented graphically in Figure 1.

Table 2: Possibilities to define $l(x)$ and $u(x)$

\begin{tabular}{|l|c|c|}
\hline Cases & $l(x)$ & $u(x)$ \\
\hline Case 1 & Use Argmin on set $N_{1}(x)$ & Use Argmin on set $N_{2}(x)$ \\
Case 2 & Use Argmin on set $N_{1}(x)$ & Use Argmax on set $N_{2}(x)$ \\
Case 3 & Use Argmax on set $N_{1}(x)$ & Use Argmax on set $N_{2}(x)$ \\
Case 4 & Use Argmax on set $N_{1}(x)$ & Use Argmin on set $N_{2}(x)$ \\
\hline
\end{tabular}

Based on Figure 1, we can conclude that: (a) Case 1 will lead to an assignment interval that concentrates on the left side of the possible assignments; (b) Case 2 will lead to the largest assignment interval; (c) Case 3 will lead to an assignment interval that concentrates on the right side of the possible assignments; and $(d)$ Case 4 will lead to the in-between and smallest interval. In the proposed approach, Case 4 has been used. We mention, however, that the other three cases can be used. Case 4 has been selected because it leads to the smallest and intermediate assignment intervals, which we will be more easily accepted by the different decision makers, especially in the presence of controversial choices.

\subsection{Step 6. Construction of collective decision table}

To finalize the construction of the collective decision table $\mathbf{S}$, some simple interval reduction rules are used for reducing the assignment intervals $I(x), \forall x \in U$, to a single class representing the value to assign to the collective 


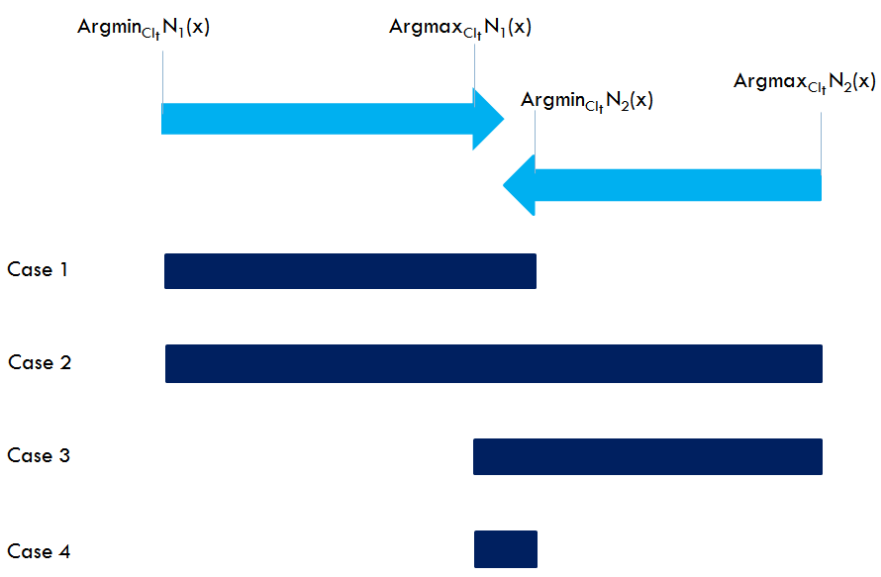

Figure 1: Definition of assignment intervals

decision attribute $E$ (as explained in Section 4). Let $I(x)=[l(x), u(x)]$ be the assignment interval for object $x \in U$ as previously defined. Two cases hold for the reduction of $I(x)$. The first case holds when $l(x)=u(x)$. Here, object $x$ is assigned to a single class and consequently we can set $g(x, E)=l(x)=u(x)$. The second case holds when $l(x) \prec u(x)$. This corresponds to the situation where object $x$ can be assigned to more than one class. To specify the value of $g(x, E)$ when the second case holds, we may apply one of the following rules to reduce the collective assignment interval $I(x)$ to a single class:

- use the "min" operator on $I(x): g(x, E)=l(x)$.

- use the "max" operator on $I(x): g(x, E)=u(x)$.

- use the "median" operator on $I(x): g(x, E)=\mu(l(x), \cdots, u(x))$.

- use the "floor" of the median value: $g(x, E)=\lfloor\mu(l(x), \cdots, u(x))\rfloor$.

- use the "ceiling" of the median value: $g(x, E)=\lceil\mu(l(x), \cdots, u(x))\rceil$.

We note that function $\mu(\cdot)$ returns the median of the values given as parameters while functions $\lfloor\cdot\rfloor$ and $\lceil\cdot\rceil$ return respectively the floor and the ceiling of the median value. It is also important to note that for quantitative variables, the median is defined as the value in the position $\frac{n+1}{2}$ if the number of values $n$ is odd and the average of the two middle values, i.e., values in positions $\frac{n}{2}$ and $\frac{n}{2}+1$, if $n$ is even. Within ordinal data, the median is generally defined as the value in the position $\frac{n}{2}$ if $n$ is even and the value in the position $\frac{n+1}{2}$ if $n$ is odd. In some cases, for ordinal values for example, the average value will be a fractional number that should be interpreted as "somewhere between $x$ and $y$ " where $x$ and $y$ are the two middle values (in positions $\frac{n}{2}$ and $\frac{n}{2}+1$, respectively). Finally, we mention that, in some cases, we can use the mode operator as follows: (i) first, use the other operators to reduce the assignment intervals, and (ii) then apply the mode operator on the results of step (i) in order to select one value.

\section{Case study}

This section presents the application of the proposed approach using real-world data.

\subsection{Decision problem}

The problem considered here concerns credit worthiness for a set of 28 European countries. The credit worthiness is generally defined as the judgment of a country current and future ability, and inclination to honor debt obligations as agreed upon. The objective of this case study is to apply the proposed approach in order to construct a common ranking scale of the considered countries based on the ranking scales established by three rating agencies, namely Fitch, Moody and S\&P. Unfruitfully, the rating agencies do not provide any information about the criteria that they 
use to establish the rankings. For the purpose of this paper, we considered a set of seven evaluation criteria, which are described in Table 3. It is clear that other criteria may be included in the analysis but our objective here is mainly to illustrate the proposed approach. Table 3 also shows the three decision attributes corresponding to the agencies Fitch, Moody and S\&P, respectively. Table 4 provides the description of the ranking scales of the different rating agencies. Based on these scales, we defined a common classification scale that is given in Table 4 (fourth column).

Table 3: List of condition criteria and decision attributes

\begin{tabular}{|l|l|l|l|l|}
\hline Code & Description & Scale type & Preference & Attribute type \\
\hline GDP & Gross Domestic Product & Continuous & Gain & Condition \\
Infl_Rate & Inflation Rate & Continuous & Cost & Condition \\
Public_Debt & Public Debt & Continuous & Cost & Condition \\
Extern_Debt & External Debt & Continuous & Cost & Condition \\
FDI & Foreign Direct Investment (as \% of GDP) & Continuous & Gain & Condition \\
CA & Current Account Balance (as \% of GDP) & Continuous & Gain & Condition \\
Unemp_Rate & Unemployment Rate & Continuous & Cost & Condition \\
rank_Fitch & Ranking by Fitch & Ordinal & Gain & Decision \\
rank_Moody & Ranking by Moody & Ordinal & Gain & Decision \\
rank_S\&P & Ranking by S\&P & Ordinal & Gain & Decision \\
\hline
\end{tabular}

Table 4: Individual and common ranking scales definition

\begin{tabular}{|c|c|c|c|c|}
\hline Fitch & Moody & S\&P & Common & Credit worthiness \\
\hline AAA & Aaa & AAA & 17 & An obligor has EXTREMELY STRONG capacity to meet its financial commitments. \\
\hline AAt & Aal & AAt & 16 & An obligor has VERY STRONG capacity to meet its financial commitments. It differs from the highest rated \\
\hline AA & $\mathrm{Aa} 2$ & AA & 15 & obligors only in small degree. \\
\hline AA- & Aa3 & AA- & 14 & \\
\hline A+ & A1 & $\mathrm{A}+$ & 13 & An obligor has STRONG capacity to meet its financial commitments but is somewhat more susceptible to the \\
\hline A & A2 & A & 12 & adverse effects of changes in circumstances and economic conditions than obligors in higher-rated categories. \\
\hline A- & A3 & A- & 11 & \\
\hline $\mathrm{BBB}+$ & Baal & $\mathrm{BBB}+$ & 10 & An obligor has ADEQUATE capacity to meet its financial commitments. However, adverse economic conditions \\
\hline BBB & Baa2 & BBB & 9 & or changing circumstances are more likely to lead to a weakened capacity of the obligor to meet its financial \\
\hline BBB- & Baa3 & BBB- & 8 & commitments. \\
\hline $\mathrm{BB}+$ & Ba1 & $\mathrm{BB}+$ & 7 & An obligor is LESS VULNERABLE in the near term than other lower-rated obligors. However, it faces major \\
\hline $\mathrm{BB}$ & $\mathrm{Ba} 2$ & $\mathrm{BB}$ & 6 & ongoing uncertainties and exposure to adverse business, financial, or economic conditions which could lead to \\
\hline BB- & $\mathrm{Ba} 3$ & BB- & 5 & the obligor's inadequate capacity to meet its financial commitments. \\
\hline $\mathrm{B}+$ & B1 & $\mathrm{B}+$ & 4 & An obligor is MORE VULNERABLE than the obligors rated 'BB', but the obligor currently has the capacity to \\
\hline $\mathrm{B}$ & B2 & $\mathrm{B}$ & 3 & meet its financial commitments. Adverse business, financial, or economic conditions will likely impair the \\
\hline B- & B3 & B- & 2 & obligor's capacity or willingness to meet its financial commitments. \\
\hline$\overline{\mathrm{CCC}}$ & Caa & $\mathrm{CCC}$ & \multirow[t]{9}{*}{1} & $\begin{array}{l}\text { An obligor is CURRENTLY VULNERABLE, and is dependent upon favourable business, financial, and economic } \\
\text { conditions to meet its financial commitments. }\end{array}$ \\
\hline $\mathrm{CC}$ & $\mathrm{Ca}$ & $\mathrm{CC}$ & & An obligor is CURRENTLY HIGHLY-VULNERABLE. \\
\hline $\mathrm{C}$ & & $\mathrm{C}$ & & $\begin{array}{l}\text { The obligor is CURRENTLY HIGHLY-VULNERABLE to nonpayment. May be used where a bankruptcy petition } \\
\text { has been filed. }\end{array}$ \\
\hline $\mathrm{D}$ & $\mathrm{C}$ & $\mathrm{D}$ & & An obligor has failed to pay one or more of its financial obligations (rated or unrated) when it became due. \\
\hline \multirow[t]{2}{*}{ Expected } & e,p & pr & & $\begin{array}{l}\text { Preliminary ratings may be assigned to obligations pending receipt of final documentation and legal opinions. } \\
\text { The final rating may differ from the preliminary rating. }\end{array}$ \\
\hline & WR & & & $\begin{array}{l}\text { Rating withdrawn for reasons including: debt maturity, calls, puts, conversions, etc., or business reasons (e.g. } \\
\text { change in the size of a debt issue), or the issuer defaults. }\end{array}$ \\
\hline unsolicited & unsolicited & unsolicited & & This rating was initiated by the ratings agency and not requested by the issuer. \\
\hline RD & & SD & & $\begin{array}{l}\text { This rating is assigned when the agency believes that the obligor has selectively defaulted on a specific issue or } \\
\text { class of obligations but it will continue to meet its payment obligations on other issues or classes of obligations } \\
\text { in a timely manner. }\end{array}$ \\
\hline NR & NR & NR & & No rating has been requested, or there is insufficient information on which to base a rating. \\
\hline
\end{tabular}

\subsection{Data set}

The data set used in this application is given in Table 5. This table provides the assessment of the considered 28 European countries in respect to the seven criteria given in Table 3 and also the ranking of these countries as established by Fitch, Moody and S\&P agencies. We note that these data has been collected from the Eurostat Website (http://ec.europa.eu/eurostat). We also note that the data in Table 5 was accessed in March 2015.

\subsection{Application}

\subsubsection{Individual approximation}

The main input for the individual approximation phase is the decision tables (Table 5). The decision objects set $U=\left\{x_{i}: i=1,2, \cdots, 28\right\}$ in this table corresponds to the considered 28 European countries. Each country is described in terms of seven criteria (designed by GDP, Infl_Rate, Public_Debt,Extern_Debt, FDI, CA and Unemp_Rate) and three decision attributes (rank_Fitch, rank_Moody, and rank_S\&P). The values of the decision 
Table 5: Information table with assignment examples (source: http://ec.europa.eu/eurostat)

\begin{tabular}{|c|c|c|c|c|c|c|c|c|c|c|c|}
\hline Object $x_{i}$ & $\begin{array}{l}\text { Country } \\
\end{array}$ & GDP & Inf_Rate & Public_Debt & Extern_Debt & FDI & $\begin{array}{l}\mathrm{CA} \\
\mathrm{A}\end{array}$ & Unemp_Rate & Rank_Fitch & Rank_Moody & Rank_S\&P \\
\hline 1 & Austria & 313,067 & 3 & 74.6 & 200 & 3.20 & 2.626 & 4.9 & AAA & Aaa & $\mathrm{AA}+$ \\
\hline 2 & Belgium & 382,692 & 0.34 & 99.6 & 266 & -0.60 & -1.841 & 8.5 & $\mathrm{AA}$ & $\mathrm{Aa} 3$ & $\mathrm{AA}$ \\
\hline 3 & Bulgaria & 39,940 & -0.8 & 17.9 & 90 & 3.50 & 1.768 & 13 & BBB- & Baa2 & $\mathrm{BB}+$ \\
\hline 4 & Croatia & 43,128 & -0.1 & 52.1 & 99 & 1.00 & 1.237 & 16 & BB & Baa3 & $\mathrm{BB}+$ \\
\hline 5 & Cyprus & 16,504 & -0.58 & 80.9 & 129 & 2.80 & -1.929 & 16.9 & B- & B3 & $\mathrm{B}+$ \\
\hline 6 & Czech Republic & 149,491 & 0.5 & 43.9 & 45 & 2.40 & -1.366 & 6.6 & A+ & $\mathrm{A} 1$ & AA- \\
\hline 7 & Denmark & 248,975 & 0.8 & 45.3 & 180 & 0.50 & 7.119 & 6.6 & AAA & Aaa & AAA \\
\hline 8 & Estonia & 18,613 & -0.4 & 6 & 87 & 3.90 & -1.207 & 7.7 & A+ & $\mathrm{A} 1$ & AA- \\
\hline 9 & Finland & 193,443 & 0.8 & 53.5 & 155 & -2 & -0.923 & 8.4 & AAA & Aaa & AAt \\
\hline 10 & France & $2,059,852$ & 0.5 & 89.9 & 182 & 0.20 & -1.433 & 10.4 & $\mathrm{AA}$ & Aal & AA \\
\hline 11 & Germany & $2,737,600$ & 0.85 & 79.9 & 142 & 1.40 & 6.863 & 5.2 & AAA & Aaa & AAA \\
\hline 12 & Greece & 182,054 & -0.7 & 161.3 & 174 & 1.20 & 0.582 & 26.8 & B & Caal & B \\
\hline 13 & Hungary & 97,948 & 0.1 & 78.6 & 115 & -3.20 & 4.120 & 7.8 & BB & $\mathrm{BB}+$ & $\mathrm{Ba} 1$ \\
\hline 14 & Ireland & 164,050 & 0.3 & 118 & $1,008.20$ & 21.50 & 6.221 & 12 & A- & Baal & A \\
\hline 15 & Italy & $1,560,024$ & 0.09 & 126.1 & 108 & 0.60 & 0.971 & 12.6 & BBB+ & Baa2 & BBB- \\
\hline 16 & Latvia & 23,372 & 0.6 & 39.2 & 146 & 2.80 & -0.806 & 11.5 & A- & Baal & A- \\
\hline 17 & Lithuania & 34,631 & 0.3 & 40.2 & 80 & 1.6 & 1.470 & 11.9 & A- & Baal & A- \\
\hline 18 & Luxembourg & 45,478 & 1 & 18.4 & 3,443 & 50.00 & 5.257 & 6.1 & AAA & Aaа & AAA \\
\hline 19 & Malta & 7,263 & 0.6 & 77 & 72 & -19.40 & 0.880 & 6.9 & A+ & A3 & BBB+ \\
\hline 20 & Netherlands & 602,658 & 0.89 & 68.7 & 73 & 3.80 & 10.203 & 7.2 & AAA & Aaа & $\mathrm{AA}+$ \\
\hline 21 & Poland & 389,695 & 2.2 & 53.8 & $72.6 \hat{\mathrm{A}}$ & 0.50 & 11.197 & 9.8 & AAA & Aaa & AAA \\
\hline 22 & Portugal & 165,690 & -0.2 & 129 & 223 & -0.90 & -1.351 & 14.8 & A- & $\mathrm{A} 2$ & A- \\
\hline 23 & Romania & 142,245 & -0.9 & 37.2 & 67 & 3.5 & 0.510 & 7.2 & $\mathrm{BB}+$ & $\mathrm{Ba} 2$ & BB \\
\hline 24 & Slovakia & 72,134 & 1 & 48.6 & 68 & 2.20 & -0.939 & 14 & & Baa3 & BBB- \\
\hline 25 & Slovenia & 35,275 & 0 & 53.2 & 47.6 & -0.90 & 6.142 & 9.6 & BBB+ & Bal & A- \\
\hline 26 & Spain & $1,022,988$ & -0.85 & 85.3 & 167 & 3.20 & 0.766 & 25.2 & BBB+ & Baa2 & BBB \\
\hline 27 & Sweden & 420,849 & 0 & 38.6 & 47 & -0.90 & 5.959 & 8 & AAA & Aaa & AAA \\
\hline 28 & United Kingdom & $1,899,098$ & 1.2 & 90 & 406 & 1.8 & -4.264 & 6.6 & $\mathrm{AA}+$ & Aal & AAA \\
\hline
\end{tabular}

attributes correspond to the rankings established by the rating agencies Fitch, Moody and S\&P. The decision tables can be represented as a collection of upward and downward unions of decision classes and approximated in the same way as with the DRSA; see Section 3. The quality of the approximations and the accuracy of classes approximations are summarized in Appendix B.1.

\subsubsection{Aggregation}

Step 1. Weighting the contribution of decision makers. We need first to quantify the contribution of decision makers into the assignment process using Equation (3) and the data given in Appendix B.1. The result is given in Table 6. At this level, it is important to remark that the accuracy of approximation of $C l_{1}^{\leq}$(for Fitch and $\mathrm{S} \& \mathrm{P}$ rating agencies) and $C l \leq \frac{\leq}{2}$ (for $\mathrm{S} \& \mathrm{P}$ rating agency) are equal to zero (see Table 6). This fact will necessary affect the assignment of objects to $C l_{1}^{\leq}$and may affect the assignment of objects to $C l_{2}^{\leq}$.

Table 6: Standardized weights

\begin{tabular}{|c|c|c|c|c|c|c|c|c|c|c|c|c|c|c|c|c|c|c|c|c|c|c|c|c|c|c|c|c|c|c|c|c|}
\hline \multirow{2}{*}{$\begin{array}{c}\text { Rating } \\
\text { agency }(k) \\
\end{array}$} & \multicolumn{16}{|c|}{$\pi_{k}\left(C l_{t}^{\geq}\right)$} & \multicolumn{16}{|c|}{$\pi_{k}\left(C l \leq \frac{\leq}{t}\right)$} \\
\hline & $t=2$ & $t=3$ & $t=4$ & $t=5$ & $t=6$ & $t=7$ & $t=8$ & $t=9$ & $t=10$ & $t=11$ & $t=12$ & $t=13$ & $t=14$ & $t=15$ & $t=16$ & $t=17$ & $t=1$ & $t=2$ & $t=3$ & $t=4$ & $t=5$ & $t=6$ & $t=7$ & $t=8$ & $t=9$ & $t=10$ & $t=11$ & $t=12$ & $t=13$ & $t=14$ & $t=15$ & $t=16$ \\
\hline & & & 0.33 & & & & & & & & 0.33 & 0.33 & 0.33 & 0.33 & & & 0 & 0.5 & & & & & & & & & 0.33 & 0.33 & & 0.33 & & 0.33 \\
\hline & 0.33 & 0.33 & 0.33 & 0.33 & 0.33 & 0.32 & 0.34 & 0.34 & 0.33 & 0.36 & 0.33 & 0.33 & 0.33 & 0.33 & 0.33 & 0.3 & & 0.5 & 0.33 & 0.33 & 0.33 & 0.21 & 0.3 & 0.33 & 0.34 & 0.38 & 0.33 & 0.33 & 0.33 & 0.33 & 0.33 & 0.33 \\
\hline S\& & 0.33 & 0.33 & 0.33 & 0.33 & 0.33 & 0.32 & 0.33 & 0.33 & 0.33 & 0.32 & 0.33 & 0.33 & 0.33 & 0.33 & 0.33 & 0.33 & & & 0.33 & 0.33 & 0.33 & 0.26 & 0.36 & 0.34 & 0.34 & 0.31 & 0.33 & 0.33 & 0.33 & 0.33 & 0.33 & 0.33 \\
\hline
\end{tabular}

Step 2. Computing concordance power. The concordance powers obtained are summarized in Appendix B.2. For illustration, we consider here the computing of $S\left(x_{14}, C l_{11}^{\leq}\right)$. Based on the lower approximations, we obtain $L\left(x_{14}, C l_{11}^{\leq}\right)=\{1,2\}$. Then, using Equation (5), we obtain: $S_{1}\left(x_{14}, C l_{11}^{\leq}\right)=0.333, S_{2}\left(x_{14}, C l_{11}^{\leq}\right)=0.333$ and $S_{3}\left(x_{14}, C l_{11}^{\leq}\right)=0$. Next, Equation (4) leads to: $\left.S\left(x_{14}, C l_{11}^{\leq}\right)=S_{1}\left(x_{14}, C l_{11}^{\leq}\right)+S_{2} x_{14}, C l_{11}^{\leq}\right)+S_{3}\left(x_{14}, C l_{11}^{\leq}\right)=$ 0.67 .

Step 3. Computing discordance power. The discordance powers obtained are summarized in Appendix B.3. For illustration, we consider here the computing of $Z\left(x_{16}, C l_{8}^{\frac{一}{8}}\right)$. Based on the boundaries, we obtain $B\left(x_{16}, C l_{8}^{\geq}\right)=$ $\{1,2,3\}$. Then, using Equation (6) and knowing that the condition $\pi_{k}\left(\mathrm{Cl}_{2}^{\geq}\right)>S\left(x_{6}, \mathrm{Cl}_{2}^{\geq}\right)$holds for all decision makers (since $S\left(x_{16}, C l_{8}^{\geq}\right)=0$ ), we obtain: $Z_{1}\left(x_{16}, C l_{8}^{\geq}\right)=\frac{1-0.33}{1-0}=0.67, Z_{2}\left(x_{16}, C l_{8}^{\geq}\right)=\frac{1-0.33}{1-0}=0.67$ and $Z_{3}\left(x_{16}, C l_{8}^{\geq}\right)=\frac{1-0.33}{1-0}=0.67$. Then, using Equation (6), we get: $Z\left(x_{16}, C l_{8}^{\geq}\right)=Z_{1}\left(x_{16}, C l_{8}^{\geq}\right) \cdot Z_{2}\left(x_{16}, C l_{8}^{\geq}\right)$. $Z_{3}\left(x_{16}, C l_{8}^{\geq}\right)=0.30$.

Step 4. Computing credibility indices. The credibility indices values are obtained simply by multiplying the concordance powers by the discordance powers (Equation (8)). The credibility indices for all decision objects are given in Appendix B.4. For instance, the credibility indices for assigning $x_{14}$ to $C l_{11}^{\leq}$and assigning $x_{16}$ to $C l_{8}^{\geq}$ 
are computed as follows: $\sigma\left(x_{14}, C l_{11}^{\leq}\right)=S\left(x_{14}, C l_{11}^{\leq}\right) \cdot Z\left(x_{14}, C l_{11}^{\leq}\right)=0.67 \cdot 1=0.67$ and $\sigma\left(x_{16}, C l_{8}^{\geq}\right)=$ $S\left(x_{16}, C l_{8}^{\geq}\right) \cdot Z\left(x_{16}, C l_{8}^{\geq}\right)=0 \cdot 0.30=0$.

Step 5. Definition of assignment intervals. Assume that the credibility threshold is equal to $\lambda=0.75$. Then, the assignment intervals can be computed using Equations (9) and (10). The application of these equations are detailed in Appendix B.5 and the obtained assignment intervals are summarized in Table 7. Let us consider the definition of the assignment interval for object $x_{15}$. Defining first the sets $N_{1}\left(x_{15}\right)$ and $N_{2}\left(x_{15}\right)$. According to the data in Appendix B.4, it is easy to see that $\sigma\left(x_{15}, C l_{t^{\prime}}\right)>0.75 \forall t^{\prime} \in\{1, \cdots, 8\}$ and $\sigma\left(x_{15}, C l_{t^{\prime \prime}}\right)<0.75 \forall t^{\prime \prime} \in\{9, \cdots, 17\}$. Accordingly, we obtain: $N_{1}\left(x_{5}\right)=\left\{C l_{1}, C l_{2}, \cdots, C l_{8}\right\}$. Similarly and based on the data in Appendix B.4, we observe that $\sigma\left(x_{15}, C l_{t^{\prime}}^{\leq}\right)>0.75 \forall t^{\prime} \in\{10, \cdots, 16\}$ and $\sigma\left(x_{15}, C l_{t^{\prime \prime}}^{\leq}\right)<0.75 \forall t^{\prime \prime} \in\{1, \cdots, 9\}$. This leads to $N_{2}\left(x_{15}\right)=\left\{C l_{10}, C l_{11}, \cdots, C l_{16}\right\}$. By Equations (9) and (10), we obtain: $l\left(x_{5}\right)=\max \left\{C l_{1}, C l_{2}, \cdots, C l_{8}\right\}=$ $C l_{8}$ and $u\left(x_{5}\right)=\min \left\{C l_{10}, C l_{11}, \cdots, C l_{16}\right\}=C l_{10}$. Finally, we obtain $I\left(x_{5}\right)=\left[C l_{8}, C l_{10}\right]$.

\begin{tabular}{|c|c|c|c|c|c|c|c|c|}
\hline \multirow[t]{2}{*}{$x_{i}$} & \multirow[t]{2}{*}{$N_{1}\left(x_{i}\right)$} & \multirow[t]{2}{*}{$N_{2}\left(x_{i}\right)$} & \multicolumn{2}{|l|}{$I(x)$} & \multicolumn{4}{|l|}{ Rule } \\
\hline & & & $l(x)$ & $u(x)$ & $\min$ & $\max$ & floor & ceiling \\
\hline 1 & $\{2,3,4,5,6,7,8,9,10,11,12,13,14,15,16\}$ & $\emptyset$ & 16 & 17 & 16 & 17 & 16 & 17 \\
\hline 2 & $\{2,3,4,5,6,7,8,9,10,11,12,13,14\}$ & $\{15,16\}$ & 14 & 15 & 14 & 15 & 14 & 15 \\
\hline 3 & 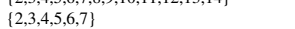 & $\{9,10,11,12,13,14,15,16\}$ & 7 & 9 & 7 & 9 & 8 & 8 \\
\hline 4 & $\{2,3,4,5,6\}$ & $\{8,9,10,11,12,13,14,15,16\}$ & 6 & 8 & 6 & 8 & 7 & 7 \\
\hline 5 & $\{2\}$ & $\{4,5,6,7,8,9,10,11,12,13,14,15,16\}$ & 2 & 4 & 2 & 4 & 3 & 3 \\
\hline 6 & $\{2,3,4,5,6,7,8,9,10,11,12,13\}$ & $\emptyset$ & 13 & 17 & 13 & 17 & 15 & 15 \\
\hline 7 & $\{2,3,4,5,6,7,8,9,10,11,12,13,14,15,16,17\}$ & $\emptyset$ & 17 & 17 & 17 & 17 & 17 & 17 \\
\hline 8 & $\left\{\begin{array}{l}\{2,3,4,5,6,7,8,9,10,11,12,13\} \\
\text { lo }\end{array}\right.$ & $\{16\}$ & 13 & 14 & 13 & 14 & 13 & 14 \\
\hline 9 & $\{2,3,4,5,6,7,8,9,10,11,12,13,14,15,16\}$ & $\emptyset$ & 16 & 17 & 16 & 17 & 16 & 17 \\
\hline 10 & $\{2,3,4,5,6,7,8,9,10,11,12,13,14,15\}$ & $\{16\}$ & 15 & 16 & 15 & 16 & 15 & 16 \\
\hline 11 & $\{2,3,4,5,6,7,8,9,10,11,12,13,14,15,16,17\}$ & $\emptyset$ & 17 & 17 & 17 & 17 & 17 & 17 \\
\hline 12 & $\emptyset$ & $\{3,4,5,6,7,8,9,10,11,12,13,14,15,16\}$ & 1 & 3 & 1 & 3 & 2 & 2 \\
\hline 13 & $\{2,3,4,5,6\}$ & $\begin{array}{l}\{7,8,9,10,11,12,13,14,15,16\} \\
\{,\end{array}$ & 6 & 7 & 6 & 7 & 6 & 7 \\
\hline 14 & $\{2,3,4,5,6,7,8,9,10\}$ & $\{12,13,14,15,16\}$ & 10 & 12 & 10 & 12 & 11 & 11 \\
\hline 15 & $\{2,3,4,5,6,7,8\}$ & $\{10,11,12,13,14,15,16\}$ & 8 & 10 & 8 & 10 & 9 & 9 \\
\hline 16 & $\{2,3,4,5,6\}$ & $\{11,12,13,14,15,16\}$ & 6 & 11 & 6 & 11 & 8 & 9 \\
\hline 17 & $\{2,3,4,5,6,7,8,9,10\}$ & $\{11,12,13,14,15,16\}$ & 10 & 11 & 10 & 11 & 10 & 11 \\
\hline 18 & $\{2,3,4,5,6,7,8,9,10,11,12,13,14,15,16,17\}$ & $\emptyset$ & 17 & 17 & 17 & 17 & 17 & 17 \\
\hline 19 & $\{2,3,4,5,6,7,8,9,10,11\}$ & $\{13,14,15,16\}$ & 11 & 13 & 11 & 13 & 12 & 12 \\
\hline 20 & $\{2,3,4,5,6,7,8,9,10,11,12,13,14,15,16\}$ & $\emptyset$ & 16 & 17 & 16 & 17 & 16 & 17 \\
\hline 21 & $\{2,3,4,5,6,7,8,9,10,11,12,13,14,15,16,17\}$ & $\emptyset$ & 17 & 17 & 17 & 17 & 17 & 17 \\
\hline 22 & $\{2,3,4,5,6,7,8,9,10,11\}$ & $\{12,13,14,15,16\}$ & 11 & 12 & 11 & 12 & 11 & 12 \\
\hline 23 & 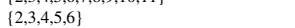 & $\{11,12,13,14,15,16\}$ & 6 & 11 & 6 & 11 & 8 & 9 \\
\hline 24 & $\{2,3,4,5,6\}$ & $\{8,9,10,11,12,13,14,15,16\}$ & 6 & 8 & 6 & 8 & 7 & 7 \\
\hline 25 & $\{2,3,4,5,6,7\}$ & $\{11,12,13,14,15,16\}$ & 7 & 11 & 7 & 11 & 9 & 9 \\
\hline 26 & $\{2,3,4,5,6,7,8,9\}$ & $\{10,11,12,13,14,15,16\}$ & 9 & 10 & 9 & 10 & 9 & 10 \\
\hline 27 & $\{2,3,4,5,6,7,8,9,10,11,12,13,14,15,16,17\}$ & $\emptyset$ & 17 & 17 & 17 & 17 & 17 & 17 \\
\hline 28 & $\{2,3,4,5,6,7,8,9,10,11,12,13,14,15,16\}$ & $\emptyset$ & 16 & 17 & 16 & 17 & 16 & 17 \\
\hline
\end{tabular}

Step 6. Construction of collective decision table. To construct the collective decision table, we need to use one of the different interval reduction rules in order to specify the values of the collective decision attribute $E$. Table 7 shows the collective decision attribute values for different interval reduction rules and for $\lambda=0.75$.

\subsubsection{Inference of collective decision rules}

At this level, the mediator should apply DRSA on the collective decision table. Table 8 provides the list of decision rules obtained for $\lambda=0.75$ and where the operator "floor" is used for interval reduction rule. Let briefly comment on some decision rules. According to Rule \#8 in Table 8, the ranking of a given country is at most $C l_{9}$ if (i) the Public Debt is greater or equal to 126.1 and (ii) the Inflation Rate is greater or equal to 0 . This rule is supported by only one decision object $\left(x_{15}\right)$ and its strength is equal to $9.09 \%$. The last Rule \#37 indicates that when (i) the GDP is greater or equal to 382692, (ii) the Inflation Rate is lower or equal to 0.34, and (iii) the Unemployment rate is lower or equal to 8.5 , then, this country should be ranked at least $C l_{13}$. Rule 37 is supported by two objects ( $x_{2}$ and $x_{27}$ ) and its strength is equal to $15.38 \%$. Finally, we observe that all these decision rules are exact.

\subsection{Discussion}

The aggregation procedure requires the definition of the credibility threshold $\lambda \in[0.5,1]$ and the selection of an interval reduction rule. In what follows, we discuss some examples illustrating the role of these parameters and the way that they can be used in practice. Let us focus first on the credibility threshold $\lambda$. Table 9 shows the assignment intervals for different values of $\lambda$. In this table, we can easily see that assignment intervals become larger by increasing the credibility threshold. This is a consequence of Properties (5) and (6) given in Appendix A.3. Indeed, the upper limit $u(x)$ of the assignment interval $I(x)$ is defined based on the values of $\sigma\left(x, C l_{t}^{\leq}\right)$. 


\begin{tabular}{|c|c|c|c|}
\hline Rule ID & Rule description & $\begin{array}{c}\text { Nb. of supporting } \\
\text { objects }\end{array}$ & Strength $(\%)$ \\
\hline Rule 1 & IF (Unemp_Rate $\geq 26.80)$ THEN (Rank $=$ At most 2) & 1 & 100 \\
\hline Rule 2 & IF $(\mathrm{CA} \leq-1.93) \& \overline{(U n e m p} \_$Rate $\left.\geq 16.9\right)$ THEN $($ Rank $=$ At most 3$)$ & 1 & 50 \\
\hline Rule 3 & IF $($ FDI $\leq-3.2) \&($ Unemp_Rate $\geq 7.8)$ THEN $($ Rank $=$ At most 6$)$ & 1 & 33.33 \\
\hline Rule 4 & IF (Unemp_Rate $\geq 14) \&(G D P \leq 72134)$ THEN $($ Rank $=$ At most 7) & 3 & 60 \\
\hline Rule 5. & IF (Unemp_Rate $\geq 13) \&(\operatorname{GDP} \leq 72134)$ THEN (Rank $=$ At most 8$)$ & 4 & 50 \\
\hline Rule 6 & $\mathrm{IF}(\mathrm{CA} \leq 0.51) \&(\overline{\mathrm{GDP}} \leq 142245) \&(\mathrm{FDI} \leq 3.5)$ THEN $($ Rank $=$ At most 8$)$ & 4 & 50 \\
\hline Rule 7 & IF (Unemp_Rate $\geq 16)$ THEN $($ Rank $=\overline{\text { At }}$ most 9$)$ & 4 & 36.36 \\
\hline Rule 8 & IF (Public_ Debt $\geq 126.1) \&($ Infl_ Rate $\geq 0)$ THEN $($ Rank $=$ At most 9$)$ & 1 & 9.09 \\
\hline Rule 9 & $\mathrm{IF}(\mathrm{GDP} \leq 35275) \&(\mathrm{FDI} \leq-0.9) \&($ Unemp_Rate $\geq 9.6) \mathrm{THEN}($ Rank $=$ At most 9$)$ & 1 & 9.09 \\
\hline Rule 10 & IF $(\mathrm{GDP} \leq 39940) \&($ Unemp_Rate $\geq 11.9)$ THEN $(\bar{R}$ ank $=$ At most 10$)$ & 3 & 25 \\
\hline Rule 11 & IF (Unemp_Rate $\geq 11.5)$ THEN $($ Rank $=$ At most 11$)$ & 11 & 78.57 \\
\hline Rule 12 & IF $(\mathrm{GDP} \leq 35275) \&(\mathrm{FDI} \leq-0.9)$ THEN $($ Rank $=$ At most 12$)$ & 2 & 13.33 \\
\hline Rule 13 & $\mathrm{IF}(\mathrm{CA} \leq 0.51) \&(\mathrm{GDP} \leq 142245)$ THEN $($ Rank $=$ At most 13$)$ & 5 & 31.25 \\
\hline Rule 14 & IF $($ CA $\leq-1.84) \&($ FDI $\leq-0.6)$ THEN $($ Rank $=$ At most 14$)$ & 1 & 5.88 \\
\hline Rule 15 & IF (Unemp_ Rate $\geq 10.40)$ THEN (Rank $=$ At most 15) & 12 & 63.16 \\
\hline Rule 16 & IF $(\mathrm{CA} \leq-1.21) \&(\mathrm{GDP} \leq 149491)$ THEN $($ Rank $=$ At most 15$)$ & 3 & 15.79 \\
\hline Rule 17 & IF $(\mathrm{CA} \leq 4.12)$ THEN $($ Rank $=$ At most 16$)$ & 20 & 86.96 \\
\hline Rule 18 & IF $($ Infl_ Rate $\geq 0.89) \&($ Public_Debt $\geq 68.75)$ THEN $($ Rank $=$ At most 16$)$ & 3 & 13.04 \\
\hline Rule 19 & IF $(\mathrm{CA} \geq 11.2)$ THEN $($ Rank $=$ At least 17$)$ & 1 & 20 \\
\hline Rule 20 & IF $(\mathrm{FDI} \geq 50)$ THEN $($ Rank $=$ At least 17$)$ & 1 & 20 \\
\hline Rule 21 & IF (GDP $\geq 2737600)$ THEN $($ Rank $=$ At least 17$)$ & 1 & 20 \\
\hline Rule 22 & IF $($ Extern_ Debt $\leq 47) \&(\mathrm{CA} \geq 5.96)$ THEN $($ Rank $=$ At least 17$)$ & 1 & 20 \\
\hline Rule 23 & IF $(\mathrm{CA} \geq 7.12) \&($ Unemp_Rate $\leq 6.6)$ THEN $($ Rank $=$ At least 17$)$ & 1 & 20 \\
\hline Rule 24 & IF $(C A \geq 6.86)$ THEN $($ Rank $=$ At least 16$)$ & 4 & 44.44 \\
\hline Rule 25 & IF (Unemp_ Rate $\leq 4.9)$ THEN $($ Rank $=$ At least 16$)$ & 1 & 11.11 \\
\hline Rule 26 & $\mathrm{IF}(\mathrm{GDP} \geq 189909 \overline{8}) \&(\mathrm{FDI} \geq 1.8) \mathrm{THEN}(\mathrm{Rank}=$ At least 16$)$ & 1 & 11.11 \\
\hline Rule 27 & IF (GDP $\geq 193443) \&($ Public_Debt $\leq 53.5)$ THEN (Rank = At least 16) & 3 & 33.33 \\
\hline Rule 28 & $\mathrm{IF}(\mathrm{CA} \geq 10.2)$ THEN $($ Rank $=$ At least 15$)$ & 2 & 18.18 \\
\hline Rule 29 & IF (Extern_ Debt $\leq 47)$ THEN (Rank = At least 15$)$ & 2 & 18.18 \\
\hline Rule 30 & IF $(\mathrm{GDP} \geq 2059852)$ THEN $($ Rank $=$ At least 15$)$ & 2 & 18.18 \\
\hline Rule 31 & $\mathrm{IF}(\mathrm{CA} \geq 10.2) \mathrm{THEN}(\mathrm{Rank}=$ At least 14$)$ & 2 & 16.67 \\
\hline Rule 32 & IF (GDP $\geq 2059852)$ THEN (Rank = At least 14) & 2 & 16.67 \\
\hline Rule 33 & IF $(\mathrm{GDP} \geq 382692) \&($ Infl_ Rate $\leq 0.34) \&($ Unemp_Rate $\leq 8.5)$ THEN $($ Rank $=$ At least 14$)$ & 2 & 16.67 \\
\hline Rule 34 & IF $(\mathrm{CA} \geq 10.2)$ THEN $($ Rank $=$ At least 13$)$ & 2 & 15.38 \\
\hline Rule 35 & IF (Public__ Debt $\leq 6)$ THEN $($ Rank $=$ At least 13) & 1 & 7.69 \\
\hline Rule 36 & IF (GDP $\geq 205985 \overline{2})$ THEN (Rank $=$ At least 13$)$ & 2 & 15.38 \\
\hline Rule 37 & IF $(\mathrm{GDP} \geq 382692) \&($ Infl_ Rate $\leq 0.34) \&($ Unemp_Rate $\leq 8.5)$ THEN (Rank = At least 13) & 2 & 15.38 \\
\hline
\end{tabular}

Property (5) sets that $\sigma\left(x, C l_{t}^{\leq}\right) \leq \sigma\left(x, C l_{t^{\prime}}\right), \forall t^{\prime} \geq t$. This means that when the value of $\lambda$ increases, the condition $\sigma\left(x, C l_{t}^{\leq}\right) \geq \lambda$ becomes more strict and the value of $u(x)$ moves in the direction of the most preferred class, i.e., $C l_{n}$. Similarly, the lower limit $l(x)$ of the assignment interval $I(x)$ is defined based on the values of $\sigma\left(x, C l_{t}^{\geq}\right)$. Property (6) sets that $\sigma\left(x, C l_{t}^{\geq}\right) \geq \sigma\left(x, C l_{t^{\prime}}^{\geq}\right), \forall t^{\prime} \geq t$. This means that when the value of $\lambda$ increases, the condition $\sigma\left(x, C l_{t}^{\geq}\right) \geq \lambda$ becomes more strict and the value of $l(x)$ moves in the direction of the least preferred class, i.e., $C l_{1}$.

Table 9: Assignment interval for different input parameters and collective decision attribute values for different input parameters

\begin{tabular}{|c|c|c|c|c|c|c|c|c|c|c|c|c|c|c|c|c|c|c|c|c|c|c|c|c|}
\hline \multirow[t]{3}{*}{$\lambda$} & \multicolumn{6}{|l|}{0.50} & \multicolumn{6}{|l|}{0.64} & \multicolumn{6}{|l|}{0.67} & \multicolumn{6}{|l|}{0.75} \\
\hline & \multicolumn{2}{|c|}{$I\left(x_{i}\right)$} & \multirow{2}{*}{\multicolumn{4}{|c|}{$\frac{\text { Rule }}{\mathrm{min}}$}} & \multicolumn{6}{|c|}{$I\left(x_{i}\right) \quad$ Rule } & \multicolumn{2}{|c|}{$I\left(x_{i}\right)$} & \multicolumn{4}{|l|}{ Rule } & \multicolumn{2}{|c|}{$I\left(x_{i}\right)$} & \multicolumn{4}{|c|}{ Rule } \\
\hline & $l\left(x_{i}\right)$ & $u\left(x_{i}\right)$ & $\min$ & $\max$ & floor & & $l\left(x_{i}\right)$ & $u\left(x_{i}\right)$ & $\min$ & $\max$ & floor & ceiling & $l\left(x_{i}\right)$ & $u\left(x_{i}\right.$ & $\min$ & $\max$ & floor & ceiling & $l\left(x_{i}\right)$ & $\mathrm{u}(\mathrm{x})$ & $\min$ & $\max$ & floor & ceiling \\
\hline$x_{i}$ & $C_{j}$ & & 1 & 2 & 3 & 4 & & & 5 & 6 & 7 & 8 & & & 9 & 10 & 11 & 12 & & & 13 & 14 & 15 & 16 \\
\hline 1 & 17 & 17 & 17 & 17 & 17 & 17 & 17 & 17 & 17 & 17 & 17 & 17 & 16 & 17 & 16 & 17 & 16 & 17 & 16 & 17 & 16 & 17 & 16 & 17 \\
\hline 2 & 15 & 15 & 15 & 15 & 15 & 15 & 15 & 15 & 15 & 15 & 15 & 15 & 14 & 15 & 14 & 15 & 14 & 15 & 14 & 15 & 14 & 15 & 14 & 15 \\
\hline 3 & 8 & 8 & 8 & 8 & 8 & 8 & 8 & 8 & 8 & 8 & 8 & 8 & 7 & 8 & 7 & 8 & 7 & 8 & 7 & 9 & 7 & 9 & 8 & 8 \\
\hline 4 & 7 & 7 & 7 & 7 & 7 & 7 & 6 & 7 & 6 & 7 & 6 & 7 & 6 & 7 & 6 & 7 & 6 & 7 & 6 & 8 & 6 & 8 & 7 & 7 \\
\hline 5 & 2 & 2 & 2 & 2 & 2 & 2 & 2 & 2 & 2 & 2 & 2 & 2 & 2 & 4 & 2 & 4 & 3 & 3 & 2 & 4 & 2 & 4 & 3 & 3 \\
\hline 6 & 13 & 14 & 13 & 14 & 13 & 14 & 13 & 14 & 13 & 14 & 13 & 14 & 13 & 17 & 13 & 17 & 15 & 15 & 13 & 17 & 13 & 17 & 15 & 15 \\
\hline 7 & 17 & 17 & 17 & 17 & 17 & 17 & 17 & 17 & 17 & 17 & 17 & 17 & 17 & 17 & 17 & 17 & 17 & 17 & 17 & 17 & 17 & 17 & 17 & 17 \\
\hline 8 & 13 & 13 & 13 & 13 & 13 & 13 & 13 & 13 & 13 & 13 & 13 & 13 & 13 & 14 & 13 & 14 & 13 & 14 & 13 & 14 & 13 & 14 & 13 & 14 \\
\hline 9 & 17 & 17 & 17 & 17 & 17 & 17 & 17 & 17 & 17 & 17 & 17 & 17 & 16 & 17 & 16 & 17 & 16 & 17 & 16 & 17 & 16 & 17 & 16 & 17 \\
\hline 10 & 15 & 15 & 15 & 15 & 15 & 15 & 15 & 15 & 15 & 15 & 15 & 15 & 15 & 16 & 15 & 16 & 15 & 16 & 15 & 16 & 15 & 16 & 15 & 16 \\
\hline 11 & 17 & 17 & 17 & 17 & 17 & 17 & 17 & 17 & 17 & 17 & 17 & 17 & 17 & 17 & 17 & 17 & 17 & 17 & 17 & 17 & 17 & 17 & 17 & 17 \\
\hline 12 & 3 & 3 & 3 & 3 & 3 & 3 & 3 & 3 & 3 & 3 & 3 & 3 & 1 & 3 & 1 & 3 & 2 & 2 & 1 & 3 & 1 & 3 & 2 & 2 \\
\hline 13 & 7 & 7 & 7 & 7 & 7 & 7 & 7 & 7 & 7 & 7 & 7 & 7 & 7 & 7 & 7 & 7 & 7 & 7 & 6 & 7 & 6 & 7 & 6 & 7 \\
\hline 14 & 11 & 11 & 11 & 11 & 11 & 11 & 11 & 11 & 11 & 11 & 11 & 11 & 10 & 12 & 10 & 12 & 11 & 11 & 10 & 12 & 10 & 12 & 11 & 11 \\
\hline 15 & 9 & 9 & 9 & 9 & 9 & 9 & 9 & 9 & 9 & 9 & 9 & 9 & 8 & 9 & 8 & 9 & 8 & 9 & 8 & 10 & 8 & 10 & 9 & 9 \\
\hline 16 & 6 & 11 & 6 & 11 & 8 & 9 & 6 & 11 & 6 & 11 & 8 & 9 & 6 & 11 & 6 & 11 & 8 & 9 & 6 & 11 & 6 & 11 & 8 & 9 \\
\hline 17 & 11 & 11 & 11 & 11 & 11 & 11 & 11 & 11 & 11 & 11 & 11 & 11 & 10 & 11 & 10 & 11 & 10 & 11 & 10 & 11 & 10 & 11 & 10 & 11 \\
\hline 18 & 17 & 17 & 17 & 17 & 17 & 17 & 17 & 17 & 17 & 17 & 17 & 17 & 17 & 17 & 17 & 17 & 17 & 17 & 17 & 17 & 17 & 17 & 17 & 17 \\
\hline 19 & 11 & 11 & 11 & 11 & 11 & 11 & 11 & 11 & 11 & 11 & 11 & 11 & 11 & 13 & 11 & 13 & 12 & 12 & 11 & 13 & 11 & 13 & 12 & 12 \\
\hline 20 & 17 & 17 & 17 & 17 & 17 & 17 & 17 & 17 & 17 & 17 & 17 & 17 & 16 & 17 & 16 & 17 & 16 & 17 & 16 & 17 & 16 & 17 & 16 & 17 \\
\hline 21 & 17 & 17 & 17 & 17 & 17 & 17 & 17 & 17 & 17 & 17 & 17 & 17 & 17 & 17 & 17 & 17 & 17 & 17 & 17 & 17 & 17 & 17 & 17 & 17 \\
\hline 22 & 11 & 11 & 11 & 11 & 11 & 11 & 11 & 11 & 11 & 11 & 11 & 11 & 11 & 12 & 11 & 12 & 11 & 12 & 11 & 12 & 11 & 12 & 11 & 12 \\
\hline 23 & 6 & 11 & 6 & 11 & 8 & 9 & 6 & 11 & 6 & 11 & 8 & 9 & 6 & 11 & 6 & 11 & 8 & 9 & 6 & 11 & 6 & 11 & 8 & 9 \\
\hline 24 & 6 & 8 & 6 & 8 & 7 & 7 & 6 & 8 & 6 & 8 & 7 & 7 & 6 & 8 & 6 & 8 & 7 & 7 & 6 & 8 & 6 & 8 & 7 & 7 \\
\hline 25 & 10 & 10 & 10 & 10 & 10 & 10 & 10 & 10 & 10 & 10 & 10 & 10 & 10 & 10 & 10 & 10 & 10 & 10 & 7 & 11 & 7 & 11 & 9 & 9 \\
\hline 26 & 9 & 9 & 9 & 9 & 9 & 9 & 9 & 9 & 9 & 9 & 9 & 9 & 9 & 9 & 9 & 9 & 9 & 9 & 9 & 10 & 9 & 10 & 9 & 10 \\
\hline 27 & 17 & 17 & 17 & 17 & 17 & 17 & 17 & 17 & 17 & 17 & 17 & 17 & 17 & 17 & 17 & 17 & 17 & 17 & 17 & 17 & 17 & 17 & 17 & 17 \\
\hline 28 & 16 & 16 & 16 & 16 & 16 & 16 & 16 & 16 & 16 & 16 & 16 & 16 & 16 & 17 & 16 & 17 & 16 & 17 & 16 & 17 & 16 & 17 & 16 & 17 \\
\hline
\end{tabular}

Let us now consider the benefits of proposing different interval reduction rules. The most important argument for proposing several interval reduction rules is the fact that these rules may apply to different decision-making situations. For instance, in decision contexts implying human health risk and human exposure to nuclear risks or 
other similar situations, the maximum rule (that picks the highest risk level here) is more appropriate. In other decision situations with a limited impact on human health, the minimum operator may be better justified. The median operator provides an intermediate value between the minimum and maximum values.

Finally, we present in Table 10 some statistics and output information. Here we just define the concepts of minimal cover, reduct and core. The minimal cover is a subset of non redundant decision rules. A reduct is a subset of attributes which can, by itself, fully characterize the knowledge in the decision table. The set of attributes which is common to all reducts is called the core. The analysis of Table 10 ensures the importance of the criterion GDP since it is often present in the core set. The next most important criterion is the Unemp_Rate followed by the criteria Public_Debt and Infl_Rate. Table 10 also shows that in all cases, a reduced set of collective decision rules in the minimal cover is generated. Finally, the quality of the classification is equal to 1 in all cases.

Table 10: Statistics and output information according to different input parameters

\begin{tabular}{|c|c|c|c|c|c|}
\hline$\lambda$ & Statistics and output information & $\begin{array}{l}\text { Rule } \\
\text { min }\end{array}$ & $\max$ & floor & ceiling \\
\hline \multirow[t]{10}{*}{0.50} & Classification quality (\%) & 1 & 1 & 1 & \\
\hline & Number of decision rules & 213 & 238 & 236 & 236 \\
\hline & Number of exact decision rules & 213 & 238 & 236 & 236 \\
\hline & $\mathrm{Nb}$. of rules in the minimal cover & 48 & 34 & 48 & 34 \\
\hline & Core & GDP & GDP & GDP & GDP \\
\hline & Reducts & 1. GDP, CA & 1. GDP, Public_ Debt & 1. GDP, CA & 1. GDP, CA \\
\hline & & 2. GDP, Public_Debt, Extern_Debt & 2. GDP, CA & 2. GDP, Public_Debt, Extern_Debt & 2. GDP, Public_ Debt, Extern_Debt \\
\hline & & 3. GDP, Public_ Debt, Unemp_Rate & 3. GDP, Infl_Rate, Unemp_ Rate & 3. GDP, Infl_ Rate, Unemp_Rate & 3. GDP, Infl_Rate, Unemp_Rate \\
\hline & & & 4. GDP, Extern__Debt, Unemp_Rate & 4. GDP, Public__ Debt, Unemp_Rate & 4. GDP, Public__ Debt, Unemp_Rate \\
\hline & & & & 5. GDP, Extern_Debt, Unemp_Rate & 5. GDP, Extern_Debt, Unemp_Rate \\
\hline \multirow[t]{10}{*}{0.64} & Classification quality (\%) & 1 & 1 & 1 & 1 \\
\hline & Number of decision rules & 215 & 238 & 242 & 238 \\
\hline & Number of exact decision rules & 215 & 238 & 242 & 238 \\
\hline & $\mathrm{Nb}$. of rules in the minimal cover & 48 & 34 & 49 & 24 \\
\hline & Core & GDP & GDP & GDP & GDP \\
\hline & Reducts & 1. GDP, CA & 1. GDP, Public_ Debt & 1. GDP, CA & 1. GDP, CA \\
\hline & & 2. GDP, Public_ Debt, Extern_ Debt & 2. GDP, $\mathrm{CA}$ & 2. GDP, Public_ Debt, Extern_ Debt & 2. GDP, Unemp_Rate \\
\hline & & 3. GDP, Public_ Debt, Unemp_ Rate & 3. GDP, Infl_ Rate, Unemp_Rate & 3. GDP, Infl_Rate, Unemp_Rate & 3. GDP, Public_ Debt, Extern_Debt \\
\hline & & & 4. GDP, Extern_ Debt, Unemp_ Rate & 4. GDP, Public__ Debt, Unemp_Rate & \\
\hline & & & & 5. GDP, Extern_ Debt, Unemp_Rate & \\
\hline \multirow[t]{13}{*}{0.67} & Classification quality (\%) & 1 & 1 & 1 & \\
\hline & Number of decision rules & 243 & 230 & 238 & 238 \\
\hline & Number of exact decision rules & 243 & 230 & 238 & 238 \\
\hline & $\mathrm{Nb}$. of rules in the minimal cover & 30 & 27 & 37 & 24 \\
\hline & Core & GDP & $\emptyset$ & GDP & GDP \\
\hline & Reducts & 1. GDP, CA & 1. GDP, CA & 1. GDP, CA & 1. GDP, CA \\
\hline & & 2. GDP, Public_ Debt, Extern__ Debt & 2. GDP, Unemp_Rate & 2. GDP, Public_Debt, Extern__ Debt & 2. GDP, Unemp_Rate \\
\hline & & 3. GDP, Public_ Debt, Unemp_Rate & 3. GDP, Public_Debt, Extern_Debt & 3. GDP, Infl_Rate, Unemp_Rate & 3. GDP, Public_Debt, Extern_Debt \\
\hline & & & 4. Infl_Rate, FDI, Unemp_Rate & 4. GDP, Public_ Debt, Unemp_Rate & \\
\hline & & & 5. Infl Rate, Public Debt, Extern Debt, FDI & 5. GDP, Extern Debt, Unemp Rate & \\
\hline & & & 6. Infl_Rate, Public_Debt, CA & & \\
\hline & & & 7. Infl_Rate, Extern_Debt, CA & & \\
\hline & & & 8. Infl_Rate, CA, Unemp_Rate & & \\
\hline \multirow[t]{13}{*}{0.75} & Classification quality (\%) & 1 & 1 & 1 & 1 \\
\hline & Number of decision rules & 251 & 238 & 295 & 243 \\
\hline & Number of exact decision rules & 251 & 238 & 295 & 243 \\
\hline & $\mathrm{Nb}$. of rules in the minimal cover & 30 & 27 & 37 & 24 \\
\hline & Core & GDP & & GDP & \\
\hline & Reducts & 1. GDP, CA & 1. GDP, CA & 1. GDP, CA & 1. GDP, CA \\
\hline & & 2. GDP, Public_ Debt, Extern_ Debt & 2. GDP, Unemp_Rate & 2. GDP, Public_ Debt, Extern_ Debt & 2. GDP, Unemp_Rate \\
\hline & & 3. GDP, Public_ Debt, Unemp_ Rate & 3. GDP, Public__ Debt, Extern_ Debt & 3. GDP, Infl_ Rate, Unemp_ Rate & 3. GDP, Public_ Debt, Extern_ Debt \\
\hline & & & 4. Infl_ Rate, FDI, Unemp_Rate & 4. GDP, Public__ Debt, Unemp_Rate & \\
\hline & & & 5. Infl_ Rate, Public_ Debt, Extern_ Debt, FDI & 5. GDP, Extern_ Debt, Unemp_Rate & \\
\hline & & & 6. Infl_Rate, Public_Debt, CA & & \\
\hline & & & 7. Infl_ Rate, Extern_ Debt, CA & & \\
\hline & & & 8. Infl_Rate, CA, Unemp_Rate & & \\
\hline
\end{tabular}

Table 11 presents the reducts and cores for individual classifications. This table shows the importance of the criteria GDP, CA and Public_Debt followed by the criteria External_Debt and Unemp_Rate. Furthermore, the analysis of Table 11 confirms the importance of the criterion GDP which is the unique criterion belonging to the core for the three agencies.

Table 11: Reducts and cores for individual classifications

\begin{tabular}{|c|c|c|c|}
\hline Agency & Fitch & Moody & S\&P \\
\hline Reducts & $\begin{array}{l}\text { 1. GDP, CA } \\
\text { 2. GDP, Public_Debt, External_Debt } \\
\text { 3. GDP, Public_Debt, Unemp_Rate }\end{array}$ & $\begin{array}{l}\text { 1. GDP, CA } \\
\text { 2. GDP, Public_-Debt, External__Debt } \\
\text { 3. GDP, Public Debt, Unemp_Rate }\end{array}$ & $\begin{array}{l}\text { 1. GDP, CA } \\
\text { 2. GDP, Public__Debt }\end{array}$ \\
\hline Core & GDP & GDP & GDP \\
\hline
\end{tabular}




\section{Additional case studies}

In this section, we provide two additional case studies illustrating the proposed approach. The first case study is related to the heat islands exposition in the Québec Metropolitan Community in Canada. The second case study concerns the management of post-accident nuclear risk in the Southern France region.

\subsection{Heat islands exposition in the QMC}

This case study was conducted as part of an action-research project whose purpose was to strengthen the resilience of the Québec Metropolitan Community (QMC), Québec, Canada, to climate change [22][43]. The objective of the second phase of this project is to assess the hot islands exposition risk at the QMC. A large set of criteria has been initially identified by the experts during this project. However, only a subset of seven criteria for which data were available for all the districts of the QMC were used to produce final risk maps. These considered criteria are: Built density (Built_Density), Built surface fraction (Built__Surface), Land-use distribution (Land_Use), Number of storeys (Number_Storeys), Electric consumption of a quarter (Elec_Consump), Electric consumption of a quarter per built surface (Elec_Consump_Surf) and Year of construction (Const__Year).

The experts have also identified a subset of 15 spatial units in the QMC - each spatial unit corresponds to an average city quarter of $250 \mathrm{~m} \times 250 \mathrm{~m}$-that have been used as learning examples. Furthermore, they jointly designed an ordinal risk scale of four levels from the lower risk (1) to the highest risk (4) to compare the spatial units of the study area. The assessment of the selected spatial units with respect to all criteria is summarized in Table 12. The experts have been organized randomly into two teams. Based on the scores of the selected spatial units with respect to all criteria and on their own expertise and knowledge of the territory, each team of experts categorizes each of the selected spatial units on the risk scale. The result of assignment process is given in Table 12 (the two last columns).

Table 12: Information table with assignment examples for heat islands exposition in the QMC

\begin{tabular}{|c|c|c|c|c|c|c|c|c|c|}
\hline Spatial Unit $x_{i}$ & Built_Density & Built_Surface & Land_Use & Number_Storeys & Elec_Consump & Elec_Consump_Surf & Const_Y Year & $T_{1}$ (Team 1$)$ & $T_{2}$ (Team 2) \\
\hline 1 & 0.5 & 42 & Commercial & 1 & 1144 & 38 & 1972 & 4 & 4 \\
\hline 2 & 0.3 & 26 & Commercial & 1 & 313 & 19 & 1973 & 4 & 4 \\
\hline 3 & 0.3 & 11 & Residential & 1.9 & 80 & 7 & 1998 & 1 & 2 \\
\hline 4 & 1.7 & 35 & Residential & 2.9 & 297 & 5 & 1929 & 3 & 2 \\
\hline 5 & 2.2 & 21 & Residential & 9.6 & 638 & 5 & 1979 & 2 & 2 \\
\hline 6 & 1.3 & 23 & Residential & 6.4 & 353 & 5 & 1985 & 2 & 3 \\
\hline 7 & 2.2 & 25 & Residential & 7.1 & 1207 & 11 & 1965 & 2 & 3 \\
\hline 8 & 2.7 & 47 & Residential & 2.6 & 667 & 7 & 1901 & 3 & 2 \\
\hline 9 & 1.6 & 37 & Residential & 2.1 & 345 & 7 & 1908 & 3 & 2 \\
\hline 10 & 0.3 & 20 & Residential & 1.3 & 120 & 6 & 1967 & 1 & 1 \\
\hline 11 & 0.4 & 17 & Residential & 1.4 & 119 & 6 & 2002 & 1 & 1 \\
\hline 12 & 0.8 & 21 & Residential & $\begin{array}{l}1.4 \\
2.9\end{array}$ & 253 & 7 & 1973 & 2 & 2 \\
\hline 13 & 0.3 & 21 & Commercial & 1 & 205 & 15 & 2006 & 4 & 4 \\
\hline 14 & 2.8 & 26 & Industrial & 1 & 318 & 0.18 & 1972 & 3 & 3 \\
\hline 15 & 2 & 42 & Industrial & 1.8 & 1060 & 1.2 & 1984 & 4 & 4 \\
\hline
\end{tabular}

We applied the proposed approach to the data in Table 12. The assignment intervals and the collective decision attribute values for different interval reduction rules are given Table 13. The list of the collective decision rules obtained for a credibility threshold $\lambda$ equal to 0.5 and by using the 'ceiling' operator are given in Table 14 . The 'ER' in this table stands for 'Exposition Risk'. The quality of approximation for this particular application is equal to 1. In addition, there are two reducts (\{Built_Surface, Elec_Consump_Surf $\}$ and \{Built_Surface, Land_Use, Number_Storeys $\})$ and one core (\{Built_Surface $\})$.

Table 13: Assignment intervals for heat islands exposition in the
\begin{tabular}{|c|ccccccc|}
\hline & $I\left(x_{i}\right)$ & \multicolumn{5}{c}{ Rule } \\
\cline { 2 - 7 } & $l\left(x_{i}\right)$ & $u\left(x_{i}\right)$ & \multicolumn{2}{c}{$\min$} & $\max$ & floor & ceiling \\
\hline$x_{i}$ & & & $C_{j}$ & 1 & 2 & 3 & 4 \\
\hline 1 & 4 & 4 & 4 & 4 & 4 & 4 \\
2 & 4 & 4 & 4 & 4 & 4 & 4 \\
3 & 1 & 2 & 1 & 2 & 1 & 2 \\
4 & 2 & 3 & 2 & 3 & 2 & 3 \\
5 & 2 & 2 & 2 & 2 & 2 & 2 \\
6 & 2 & 3 & 2 & 3 & 2 & 3 \\
7 & 2 & 3 & 2 & 3 & 2 & 3 \\
8 & 3 & 3 & 3 & 3 & 3 & 3 \\
9 & 2 & 3 & 2 & 3 & 2 & 3 \\
10 & 1 & 1 & 1 & 1 & 1 & 1 \\
11 & 1 & 1 & 1 & 1 & 1 & 1 \\
12 & 2 & 2 & 2 & 2 & 2 & 2 \\
13 & 4 & 4 & 4 & 4 & 4 & 4 \\
14 & 3 & 3 & 3 & 3 & 3 & 3 \\
15 & 4 & 4 & 4 & 4 & 4 & 4 \\
\hline
\end{tabular}


Table 14: Collective decision rules for heat islands exposition in the QMC

\begin{tabular}{|c|c|c|c|}
\hline Rule ID & Decision rule description & $\mathrm{Nb}$. of supporting & Strength $(\%)$ \\
\hline Rule 1 & IF (Elec_Consump $\leq 120) \&($ Number_Storeys $\leq 1.4)$ THEN ER $=$ At most 1 & 2 & 100 \\
\hline Rule 2 & IF (Built_Surface $\leq 21) \&($ Elec_Consump_Surf $<=5$ ) THEN ER $=$ Atmost 2 & 1 & 20 \\
\hline Rule 3 & IF $($ Elec_Consump $\leq 253) \&($ Elec_C Consump_Surf $\leq 7)$ THEN ER $=$ At most 2 & 4 & 80 \\
\hline Rule 4 & IF (Land__ Use=2) THEN ER = At most 3 & 10 & 90.91 \\
\hline Rule 5 & IF (Elec_ Consump__Surf $\leq 0.18$ ) THEN ER $=$ At most 3 & 1 & 9.09 \\
\hline Rule 6 & IF (Elec_Consump_Surf $\geq 15$ ) THEN ER $=$ At least 4 & 3 & 75 \\
\hline Rule 7 & IF (Land_Use $=3$ ) \& (Elec_Consump_Surf $\geq 1.2)$ THEN ER $=$ At least 4 & 1 & 25 \\
\hline Rule 8 & IF (Built__Surface $\geq 23$ ) THEN ER = At least 3 & 9 & 90 \\
\hline Rule 9 & IF (Elec_C Consump $\geq 205$ ) THEN ER $=$ At least 2 & 12 & 92.31 \\
\hline Rule 10 & IF (Elec_Consump_S Surf $\geq 7$ ) THEN ER $=$ At least 2 & 8 & 61.54 \\
\hline
\end{tabular}

\subsection{Management of post-accident nuclear risk}

The problem considered in this case study concerns the management of post-accident nuclear risk in the Southern France region. This evaluation was conducted during the PRIME project, which was supervised by the French Institute for Radioprotection and Nuclear Safety. A full description of the project is available in [49]. The studied zone covers a radius of some fifty kilometers around three nuclear sites in the lower Rhône Valley (the Cruas, Tricastin-Pierrelatte and Marcoule sites). The objective of PRIME is to develop-conjointly with the decision makers, the stakeholders and representatives of the territory - a multicriteria evaluation approach in order to permit the analysis and characterization of the contaminated territory, which will be useful for the risk managers. The evaluation approach should associate to each district of the study area a degree representing the risk on this district of a nuclear accident resulting in releases into the atmosphere. For this purpose, a scale of six levels (0: normal situation; 1: very minor impact; 2 : minor impact; 3 : moderate impact; 4: major impact; and 5: major and long-lasting negative impact) has been adopted by the PRIME working team.

For the purpose of this paper, only a subset of data is used for illustration. The problem discussed here involves 18 decision objects (corresponding to a subset of the districts of the study area) selected from 492 decision objects, 7 evaluation criteria and three decision makers denoted CM, PP and CAL, respectively. The criteria considered in this paper are: Radioecological vulnerability of agricultural area (Agri_ Vul), Radioecological vulnerability of forest area (Forest_Vul), Radioecological vulnerability of urban area (Urban_Vul), Real estate vulnerability (RealEstate_Vul), Tourism vulnerability (Tour_Vul), Economic vulnerability of companies (Eco__Vul) and Employment vulnerability (Emp_Vul). The evaluation of the decision objects in respect to these criteria is given in Table 15. We note that the decision table given in Table 15 contains two inconsistent objects, namely \#4, and \#6. They are inconsistent, because \#4 has better evaluations on all three attributes than \#6 but a worse global score.

Table 15: Information table with assignment examples for the post-accident nuclear risk assessment

\begin{tabular}{|c|cccccccccc|c|}
\hline District $x_{i}$ & Agri_Vul & Forest_Vul & Urban_Vul & RealEstate_Vul & Tour_Vul & Eco__Vul & Emp_Vul & CM & PP & CAL \\
\hline 1 & 4 & 5 & 5 & 5 & 4 & 1 & 1 & 4 & 4 & 5 \\
2 & 4 & 5 & 5 & 5 & 4 & 2 & 2 & 4 & 4 & 5 \\
3 & 4 & 5 & 5 & 5 & 4 & 2 & 1 & 4 & 4 & 5 \\
4 & 4 & 5 & 5 & 5 & 4 & 3 & 1 & 5 & 4 & 5 \\
5 & 3 & 2 & 2 & 4 & 4 & 2 & 0 & 3 & 2 & 3 \\
6 & 1 & 1 & 1 & 2 & 4 & 1 & 0 & 0 & 0 & 1 \\
7 & 2 & 2 & 1 & 2 & 4 & 1 & 0 & 3 & 2 \\
8 & 1 & 2 & 1 & 2 & 2 & 1 & 0 & 0 & 0 & 1 \\
9 & 3 & 2 & 2 & 4 & 4 & 2 & 0 & 3 & 2 & 2 \\
10 & 3 & 3 & 3 & 4 & 4 & 1 & 0 & 3 & 2 & 3 \\
11 & 3 & 3 & 3 & 4 & 4 & 1 & 0 & 3 & 2 & 3 \\
12 & 3 & 3 & 2 & 4 & 4 & 1 & 0 & 3 & 2 \\
13 & 3 & 2 & 2 & 4 & 4 & 1 & 0 & 2 & 2 \\
14 & 2 & 2 & 2 & 4 & 4 & 1 & 0 & 2 & 1 \\
15 & 2 & 2 & 1 & 4 & 3 & 1 & 0 & 2 & 1 \\
16 & 2 & 2 & 1 & 4 & 4 & 1 & 0 & 2 & 1 \\
17 & 1 & 1 & 1 & 2 & 4 & 1 & 0 & 3 & 3 \\
18 & 1 & 1 & 0 & 1 & 4 & 1 & 0 & 3 & 3 \\
\hline
\end{tabular}

Table 16 shows the assignment intervals and the collective decision attribute values for different values of credibility threshold $\lambda$ and different interval reduction rules. Table 17 provides the list of decision rules obtained for $\lambda=0.70$ and where the operator "floor" is used for interval reduction rule.

\section{Comparison of results}

The final classifications generated by the DRSAfG may differ according to the input data and parameters. The objective of this section is to provide a formal way to compare the different final classifications using a series of well-known non-parametric statistics, namely Kendall's $\tau$, Spearman's $\rho$, Cohen's $\kappa$, Kendall's $W$ and Fless's $\kappa$. 
Table 16: Assignment intervals for the post-accident nuclear risk assessment

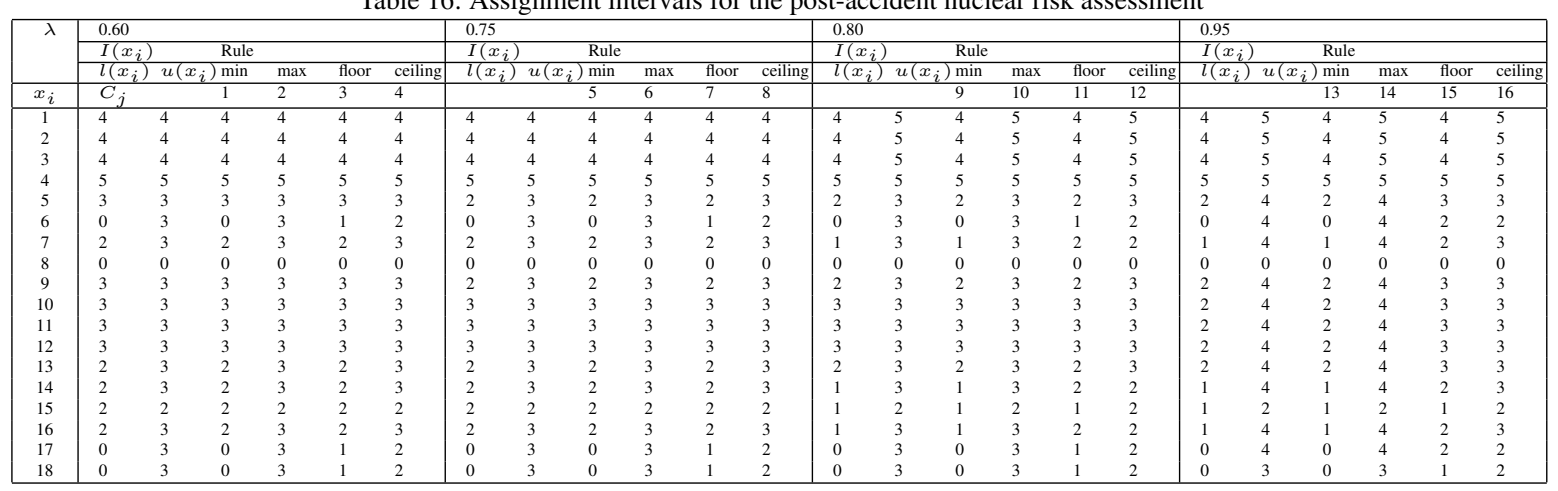

\begin{tabular}{|c|c|c|c|}
\hline Rule ID & Rule description & $\begin{array}{c}\begin{array}{c}\text { Nb. of supporting } \\
\text { objects }\end{array} \\
\end{array}$ & Strength $(\%)$ \\
\hline Rule 1 & IF (Tour_ Vul $\leq 2)$, THEN $($ Risk $=0)$ & 1 & 100 \\
\hline Rule 2 & IF (Agri__ Vul $\leq 1)$, THEN $($ Risk $=$ At most 1$)$ & 4 & 100 \\
\hline Rule 3 & IF $($ Forest - Vul $\leq 1)$, THEN $($ Risk $=$ At most 1$)$ & 3 & 75 \\
\hline Rule 4 & IF (Forest - Vul $\leq 2)$, THEN (Risk $=$ At most 2$)$ & 11 & 100 \\
\hline Rule 5 & IF (Agri_ Vul $\leq 3)$, THEN (Risk = At most 3 ) & 14 & 100 \\
\hline Rule 6 & IF (Urban_ Vul $\leq 2)$, THEN $($ Risk $=$ At most 3$)$ & 12 & 85.71 \\
\hline Rule 7 & IF (RealEstate_- Vul $\leq 4)$, THEN $($ Risk $=$ At most 3$)$ & 14 & 100 \\
\hline Rule 8 & IF (Emp_Vul $\leq 0), \overline{\text { THEN }}($ Risk $=$ At most 3$)$ & 14 & 100 \\
\hline Rule 9 & IF (Forest_ Vul $\leq 3)$, THEN (Risk $=$ At most 3 ) & 14 & 100 \\
\hline Rule 10 & IF (Urban_ Vul $\leq 3)$, THEN (Risk $=$ At most 3$)$ & 14 & 100 \\
\hline Rule 11 & IF $\left(\right.$ Eco $\_$Vul $\left.\leq 2\right)$, THEN $($ Risk $=$ At most 4$)$ & 17 & 100 \\
\hline Rule 12 & IF (Eco_ Vul $\geq 3)$, THEN (Risk = At least 5) & 1 & 100 \\
\hline Rule 13 & IF $($ Agri - Vul $\geq 4)$, THEN $($ Risk $=$ At least 4$)$ & 4 & 100 \\
\hline Rule 14 & IF (Forest $\_$Vul $\geq 5$ ), THEN (Risk $=$ At least 4 ) & 4 & 100 \\
\hline Rule 15 & IF (Urban_Vul $\geq 5)$, THEN $($ Risk $=$ At least 4$)$ & 4 & 100 \\
\hline Rule 16 & IF (RealEstate - Vul $\geq 5)$, THEN $($ Risk $=$ At least 4$)$ & 4 & 100 \\
\hline Rule 17 & IF (Emp_Vul $\geq 1)$, THEN $($ Risk $=$ At least 4$)$ & 4 & 100 \\
\hline Rule 18 & IF (Forest_ Vul $\geq 3$ ), THEN $($ Risk $=$ At least 3 ) & 7 & 100 \\
\hline Rule 19 & IF (Agri - Vul $\geq 2)$, THEN $($ Risk $=$ At least 2$)$ & 14 & 100 \\
\hline Rule 20 & IF (Forest_ Vul $\geq 2) \wedge($ Tour_ Vul $\geq 4)$, THEN $($ Risk $=$ At least 2$)$ & 13 & 92.86 \\
\hline Rule 21 & IF (RealEstate_ Vul $\geq 4)$, THEN $($ Risk $=$ At least 2$)$ & 13 & 92.86 \\
\hline Rule 22 & IF $($ Forest_Vul $\geq 2) \wedge($ Tour_Vul $\geq 3)$, THEN $($ Risk $=$ At least 2$)$ & 14 & 100 \\
\hline Rule 23 & IF $\left(\right.$ Tour $\_$Vul $\left.\geq 3\right)$, THEN $($ Risk $=$ At least 1$)$ & 17 & 100 \\
\hline
\end{tabular}

\subsection{Comparing final classifications}

The main input for the DRSAfG is the individual classifications specified by the different decision makers. Intuitively, we prefer that the final collective classification produced by the aggregation procedure reproduces perfectly these classifications. This is often unreachable since decision makers generally provide different assignments. A more reasonable objective is to obtain a final classification that reproduces "at best" the individual classifications. Let $C_{i}(i=1, \cdots, k)$ be an individual classification provided by the $i$ th $(i=1, \cdots, k)$ decision maker and $C_{j}$ a final ranking generated by the aggregation procedure. The first possible comparison is obtained by a direct application of the Kendall's $\tau$, Spearman's $\rho$ and/or Cohen's $\kappa$ coefficients to evaluate the agreement between the final rankings and each of the initial rankings.

The Kendall's $\tau$ coefficient is a metric that counts the number of pairwise disagreements between two ranking lists. The Spearman's $\rho$ measures the statistical dependence between two variables and assesses how well the relationship between two variables can be described using a monotonic function. The Cohen's $\kappa$ is a measurement of concordance or agreement between two rankings. There are two ways of calculating Cohen's $\kappa$ : unweighted and weighted. The weighted $\kappa$ is more appropriate to variables having more than two categories. The Kendall's $\tau$, Spearman's $\rho$ and Cohen's $\kappa$ are designed to compare two given rankings. In addition, these three statistics accept ordinal data and can deal with ties. In the rest of this paper, we design by $\tau\left(C_{j}, C_{i}\right), \rho\left(C_{j}, C_{i}\right)$ and $\kappa\left(C_{j}, C_{i}\right)$ the value of the Kendall's $\tau$, Spearman's $\rho$ and Cohen's $\kappa$ coefficients relative to a final ranking $C_{j}$ and an initial ranking $C_{i}$.

Let $\theta \in\{\tau, \rho, \kappa\}$. Then, we can easily establish that if $\theta\left(C_{j}, C_{i}\right) \geq \theta\left(C_{j^{\prime}}, C_{i}\right)$ then $C_{j}$ is more close to individual classification $C_{i}$ than $C_{j^{\prime}}$. In other words, $C_{j}$ reproduces better the individual classification $C_{i}$ than does $C_{j^{\prime}}$.

Comparison rule 1. Let $C_{j}$ and $C_{j^{\prime}}$ be two collective classifications and let $C_{i}$ be an individual classification. 
[Post print version, please cite as] Chakhar Salem, Ishizaka Alessio, Labib Ashraf, Saad Inès, Dominance-based Rough Set Approach for Group Decisions, European Journal of Operational Research, advance online publications, doi:10.1016/j.ejor.2015.10.060

Then, if $\theta\left(C_{j}, C_{i}\right) \geq \theta\left(C_{j^{\prime}}, C_{i}\right)$, then $C_{j}$ reproduces better the individual classification $C_{i}$ than $C_{j^{\prime}}$. Classification $C_{j}$ should be selected.

The rule above applies for one final classification and one initial classification. It is more appropriate to compute the closeness of a final classification to all the initial classifications taken together. We can compute the closeness of a collective classification $C_{j}$ to a set of individual classifications $C=\left\{C_{1}, C_{2}, \cdots, C_{k}\right\}$ as follows:

$$
E_{\theta}\left(C_{j}, C\right)=\sum_{C_{i} \in C} \theta\left(C_{j}, C_{i}\right)
$$

where $\theta \in\{\tau, \rho, \kappa\}$. Then, we can easily establish that if $E_{\theta}\left(C_{j}, C\right) \geq E_{\theta}\left(C_{j^{\prime}}, C\right)$ then $C_{j}$ is more close to individual classifications set $C=\left\{C_{1}, \cdots, C_{k}\right\}$ than $C_{j^{\prime}}$. In other words, $C_{j}$ reproduces better the individual classifications than does $C_{j^{\prime}}$.

Comparison rule 2. Let $C_{j}$ and $C_{j^{\prime}}$ be two collective classifications obtained from the same set of individual classifications set $C=\left\{C_{1}, \cdots, C_{k}\right\}$. Then, if $E_{\theta}\left(C_{j}, C\right) \geq E_{\theta}\left(C_{j^{\prime}}, C\right)$, then $C_{j}$ reproduces better the individual classifications than $C_{j^{\prime}}$. Classification $C_{j}$ should be selected.

The scores given by Equation (11) for different statistics can be combined in order to identify the best collective classification based on the different statistics taken together:

$$
E\left(C_{j}, C\right)=E_{\tau}\left(C_{j}, C\right)+E_{\rho}\left(C_{j}, C\right)+E_{\kappa}\left(C_{j}, C\right) .
$$

We can easily establish that if $E\left(C_{j}, C\right) \geq E\left(C_{j^{\prime}}, C\right)$ then $C_{j}$ is more close to individual classifications set $C=\left\{C_{1}, \cdots, C_{k}\right\}$ than $C_{j^{\prime}}$. In other words, $C_{j}$ reproduces better the individual classifications set $C$ than does $C_{j^{\prime}}$.

Comparison rule 3. Let $C_{j}$ and $C_{j^{\prime}}$ be two collective classifications obtained from the same set of individual classifications $C=\left\{C_{1}, \cdots, C_{k}\right\}$. Then, if $E\left(C_{j}, C\right) \geq E\left(C_{j^{\prime}}, C\right)$, then $C_{j}$ reproduces better the individual classifications in $C$ than $C_{j^{\prime}}$. Classification $C_{j}$ should be selected.

A more appropriate way to compare a given final classification in respect to all the initial rankings consists in the use of the Kendall's $W$ and/or Fleiss's $\kappa$ statistics. The Kendall's $W$ (also known as Kendall's coefficient of concordance) is used for assessing agreement among multiple rankings. The Fless's $\kappa$ is an extension of Cohen's $\kappa$ to evaluate concordance or agreements between multiple rankings. The Kendall's $W$ and Fless's $\kappa$ are devoted to compare at least three different rankings. In addition, both of them accept ordinal data and can deal with ties.

Let $C=\left\{C_{1}, C_{2}, \cdots, C_{k}\right\}$ be a set of $k$ initial rankings and let $C_{j}$ be a final ranking generated by the aggregation procedure. Then, the statistics Kendall's $W$ and Fleiss's $\kappa$ can be directly used to measure the closeness of the final ranking $C_{j}$ to the set of initial rankings $C=\left\{C_{1}, C_{2}, \cdots, C_{k}\right\}$. In the rest of this paper, we design by $W\left(C_{j}, C\right)$ and $F\left(C_{j}, C\right)$ the comparison of the final ranking $C_{j}$ to initial rankings set $C=\left\{C_{1}, C_{2}, \cdots, C_{k}\right\}$ using Kendall's $W$ and Fleiss's $\kappa$ statistics, respectively.

Let $\phi \in\{W, F\}$. Then, we can easily establish that if $\phi\left(C_{j}, C\right) \geq \phi\left(C_{j^{\prime}}, C\right)$ then $C_{j}$ is more close to the individual classifications set $C$ than $C_{j^{\prime}}$. In other words, $C_{j}$ reproduces better the individual classifications set $C$ than does $C_{j^{\prime}}$.

Comparison rule 4. Let $C_{j}$ and $C_{j^{\prime}}$ be two collective classifications and let $C=\left\{C_{1}, C_{2}, \cdots, C_{k}\right\}$ be a set of $k$ initial rankings. Then, if $\phi\left(C_{j}, C\right) \geq \phi\left(C_{j^{\prime}}, C\right)$, then $C_{j}$ reproduces better the individual classification set $C$ than $C_{j^{\prime}}$. Classification $C_{j}$ should be selected.

The scores given by $W\left(C_{j}, C\right)$ and $F\left(C_{j}, C\right)$ can be combined in order to identify the best collective classification based on the statistics Kendall's $W$ and Fleiss's $\kappa$ taken together:

$$
E^{\prime}\left(C_{j}, C\right)=W\left(C_{j}, C\right)+F\left(C_{j}, C\right) .
$$

We can easily establish that if $E^{\prime}\left(C_{j}, C\right) \geq E^{\prime}\left(C_{j^{\prime}}, C\right)$ then $C_{j}$ is more close to individual classifications set $C=\left\{C_{1}, \cdots, C_{k}\right\}$ than $C_{j^{\prime}}$. In other words, $C_{j}$ reproduces better the individual classifications than does $C_{j^{\prime}}$. 
Comparison rule 5. Let $C_{j}$ and $C_{j^{\prime}}$ be two collective classifications obtained from the same set of individual classifications $C=\left\{C_{1}, \cdots, C_{k}\right\}$. Then, if $E^{\prime}\left(C_{j}, C\right) \geq E^{\prime}\left(C_{j^{\prime}}, C\right)$, then $C_{j}$ reproduces better the individual classifications than $C_{j^{\prime}}$. Classification $C_{j}$ should be selected.

\subsection{Illustration}

For illustration, we provide in Table 18 the values of $\theta\left(C_{j}, C_{i}\right), E_{\theta}\left(C_{j}, C_{i}\right), E\left(C_{j}, C\right), \phi\left(C_{j}, C_{i}\right)$ and $E^{\prime}\left(C_{j}, C\right)$ with $\theta \in\{\tau, \rho, \kappa\}, \phi \in\{W, F\}, j \in\{1,2, \cdots, 16\}$ and $i=1,2,3$, for the initial and final classifications given in Table 5 and Table 9, respectively. The values of Kendall $\tau$ coefficient are computed using the Web-based and Free Statistics Software [66], the Spearman $\rho$ and the Cohen's $\kappa$, Kendall's $W$ and Fleiss's $\kappa$ coefficients are computed using the Web-based StatsToDo Software [59]. In Table 18, the minimum values (corresponding to worst final classification) are underlined while the maximum values (corresponding to best final classification) are in boldface.

The Kendall's $\tau$ coefficient is in the range [-1,1]. If the agreement between the two rankings is perfect (i.e., the two rankings are the same) the coefficient has value 1. If the disagreement between the two rankings is total (i.e., one ranking is the reverse of the other) the coefficient has value -1. If two rankings are independent, then we would expect the coefficient to be approximately zero. The Spearman's $\rho$ coefficient is in the range $[-1,1]$. A positive Spearman correlation coefficient indicates that both rankings vary in the same direction. A negative Spearman $\rho$ coefficient indicates a monotone decreasing relationship between the two rankings. A Spearman $\rho$ coefficient of zero indicates that there is no tendency between the two rankings. Conventionally, a Kappa of $<0.2$ is considered poor agreement, 0.21-0.4 fair, 0.41-0.6 moderate, 0.61-0.8 strong, and more than 0.8 near complete agreement. As shown in Table 18, the values of Kendall's $\tau$ and Spearman's $\rho$ coefficients all tend towards 1. We can conclude that there is a high level of agreement between initial and final rankings. The values of Cohen's $\kappa$ in Table 18 are all greater than 0.8 , indicating a near complete agreement between each of initial and final rankings.

Table 18: Kendall's $\tau$, Spearman's $\rho$, Cohen's $\kappa$, Kendall's $W$ and Fleiss's $\kappa$ coefficients

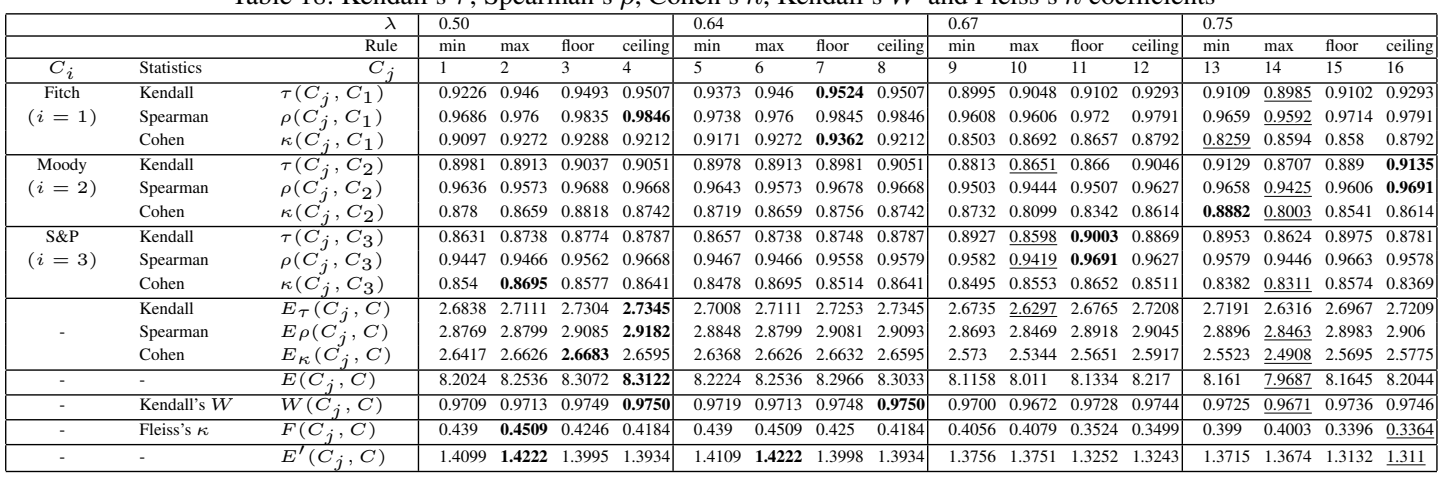

The Kendall's $W$ coefficient ranges from 0 (no agreement) to 1 (complete agreement). Intermediate values of Kendall's $W$ indicate a greater or lesser degree of unanimity among the various rankings. A negative Fleiss's kappa indicates poor agreement. A Fleiss's $\kappa$ in the range [0,1] indicates an increasing levels of agreement. A Fleiss's $\kappa$ greater than 0.8 indicates a near perfect agreement. The values of Kendall's $W$ coefficient in Table 18 tend towards 1. We can conclude that according to Kendall's $W$ coefficient, there is a high level of agreement between the initial and the final rankings. The values of Fleiss's $\kappa$ show a moderate agreement between the initial and the final rankings.

We conducted the same comparison exercise presented above with the two other case studies given in Section 7.1 and in Section 7.2, respectively. A summary of this comparison is given in Appendix C.

\section{Conclusion and future work}

We proposed an approach, called DRSAfG, designed to handle multiple decision makers in a way that leads to one compromise solution. The DRSAfG is structured into three phases. Firstly, the individual approximation of the knowledge of each decision maker into lower and upper approximation sets. Secondly, the outputs of the first phase are aggregated into a collective decision table. Thirdly, the inference of collective decision rules. The approach is illustrated through a series of case studies relative to credit worthiness of a set of European countries, heat islands 
exposition in the Québec Metropolitan Community in Canada and the management of post-accident nuclear risk in the Southern France region.

The DRSAfG approach presented in this paper addresses appropriately the shortcomings of existing works that we mentioned in Section 1: (i) it uses a mixed aggregation strategy to combine the judgements and perspectives of involved decision makers, which avoids the problems of input or output strategies used alone; (ii) it measures objectively the "powers" of decision makers based on their preferences; (iii) it combines the preference and knowledge of decision makers based on the majority principle and veto effect, which leads to more consensual and fairer decisions; (iv) it uses a reduced set of decision objects in order to extract and generalize the preferences of the decision makers thus minimizing their cognitive effort; and (v) it is enhanced with several tools permitting to compare final results obtained using different input parameters, which substantially improve the effectiveness of the decision making process and the successful implementation of the final solution.

Three main directions of research should be investigated in the future. The first direction concerns the use of the VC-DRSA [32] or the method of [5] instead of the conventional DRSA. The second direction of research is to use the input oriented strategy to combine the judgments and perspectives of decision makers by using some appropriate rules (such as maxmin, minmax or leximax criteria) or a distance/measure function and then apply the DRSA to infer the collective decision rules. The third direction of research concerns the exploration of the decision rule-related information to collectivity classify the decision objects by collecting the individual decision rules obtained from different decision makers and then the use of some appropriate metrics to remove redundant and contradictory decision rules.

\section{Acknowledgements}

The authors would like to thank the Editor-in-Chief and the anonymous reviewers for their valuable and very helpful comments that permitted to improve the quality of the paper. The authors would also like to thank Dr Yaseen Ghulam for his help on the criteria identification, data collection and rating definitions used in the case study of Section 6 and Dr Renatas Kizys for his advice concerning the list of criteria used in the case study of Section 6. The case study in Section 7.1 has been conduced during the Climate Change and Urban Transformation Research-Action Project supported by Ouranos Consortium (http://www.ouranos.ca/en/), Natural Resources Canada (http://www.nrcan.gc.ca/) and the Fonds Vert Québec (http://www.mddep.gouv.qc.ca/). The first author would like to thank his previous colleagues in The Centre for Research in Regional Planning and Development (University of Laval, Québec City, QC, Canada) and members of the Action-Research Project, especially Dr Geneviève Cloutier, Dr Christelle Legay, Dr Catherine Dubois, Prof Florent Joerin, Prof Manuel J. Rodriguez, Marc Ferreri, Martial Labarthe and Dominique Viens.

\section{References}

[1] Abastante, F., Bottero, M., Greco, S., Lami, I., 2014. Addressing the location of undesirable facilities through the dominance-based rough set approach. Journal of Multi-Criteria Decision Analysis 21 (1-2), 3-23.

[2] Almeida-Dias, J., Figueira, J., Roy, B., 2010. Electre Tri-C: A multiple criteria sorting method based on characteristic reference actions. European Journal of Operational Research 204 (3), 565-580.

[3] Almeida-Dias, J., Figueira, J., Roy, B., 2012. A multiple criteria sorting method where each category is characterized by several reference actions: The Electre Tri-nC method. European Journal of Operational Research 217 (3), 567-579.

[4] Blasco, H., Blaszczyński, J., Billaut, J., Nadal-Desbarats, L., Pradat, P., Devos, D., Moreau, C., Andres, C., Emond, P., Corcia, P., Slowiński, R., 2015. Comparative analysis of targeted metabolomics: Dominancebased rough set approach versus orthogonal partial least square-discriminant analysis. Journal of Biomedical Informatics 53, 291-299.

[5] Blaszczyński, J., Greco, S., Slowiński, R., 2007. Multi-criteria classification - a new scheme for application of dominance-based decision rules. European Journal of Operational Research 181 (3), 1030-1044.

[6] Blaszczyński, J., Greco, S., Slowiński, R., 2012. Inductive discovery of laws using monotonic rules. Engineering Applications of Artificial Intelligence 25 (2), 284-294. 
[Post print version, please cite as] Chakhar Salem, Ishizaka Alessio, Labib Ashraf, Saad Inès, Dominance-based Rough Set Approach for Group Decisions, European Journal of Operational Research, advance online publications, doi:10.1016/j.ejor.2015.10.060

[7] Blaszczyński, J., Slowiński, R., Szelạg, M., 2012. Induction of ordinal classification rules from incomplete data. In: Yao, J., Yang, Y., Slowiński, R., Greco, S., Li, H., Mitra, S., Polkowski, L. (Eds.), Rough Sets and Current Trends in Computing. Vol. 7413 of Lecture Notes in Computer Science. Springer Berlin Heidelberg, pp. 56-65.

[8] Bregar, A., Györkös, J., Jurič, M., 2008. Interactive aggregation/disaggregation dichotomic sorting procedure for group decision analysis based on the threshold model. Informatica 19 (2), 161-190.

[9] Brigui-Chtioui, I., Saad, I., 2011. A multi-agent approach for collective decision making in knowledge management. Group Decision and Negotiation 20 (1), 19-37.

[10] Cai, F.-L., Liao, X., Wang, K.-L., 2012. An interactive sorting approach based on the assignment examples of multiple decision makers with different priorities. Annals of Operations Research 197, 87-108.

[11] Chakhar, S., Pusceddu, C., 2013. Decision tables aggregation in rough sets approximation. In: BoonthumDenecke, C., Youngblood, G. (Eds.), Proceedings of the Twenty-Sixth International Florida Artificial Intelligence Research Society Conference, FLAIRS 2013, St. Pete Beach, Florida. May 22-24, 2013. AAAI Press, pp. 633-636.

[12] Chakhar, S., Pusceddu, C., Saad, I., 2012. Evaluating post-accident nuclear risk by coupling GIS and rough sets theory. In: Campagna, M., Montis, A. D., Isola, F., Lai, S., Pira, C., Zoppi, C. (Eds.), Planning Support Tools: Policy Analysis, Implementation and Evaluation, Proceedings of the Seventh International Conference on Informatics and Urban and Regional Planning (INPUT 2012). May 10-12, 2012. Cagliari, Italy, pp. $223-235$.

[13] Chakhar, S., Saad, I., 2012. Dominance-based rough set approach for groups in multicriteria classification. Decision Support Systems 54 (1), 372-380.

[14] Chakhar, S., Saad, I., 2014. Incorporating stakeholders' knowledge in group decision-making. Journal of Decision Systems 23 (1), 113-126.

[15] Chen, Y., Kilgour, D., Hipel, K., 2012. A decision rule aggregation approach to multiple criteria-multiple participant sorting. Group Decision and Negotiation 21, 727-745.

[16] Cook, W., 2006. Distance-based and ad hoc consensus model in ordinal preference ranking with intensity of preference. European Journal of Operational Research 172 (2), 369-385.

[17] Damart, S., Dias, L., Mousseau, V., 2007. Supporting groups in sorting decisions: Methodology and use of a multicriteria aggregation/disaggregation DSS. Decision Support Systems 43 (4), 1464-1475.

[18] Dembczynski, K., Greco, S., Kotlowski, W., Slowiński, R., 2007. Statistical model for rough set approach to multicriteria classification. In: Kok, J., Koronacki, J., Lopez de Mantaras, R., Matwin, S., Mladenic, D., Skowron, A. (Eds.), Knowledge Discovery in Databases: PKDD 2007. Vol. 4702 of Lecture Notes in Computer Science. Springer Berlin Heidelberg, pp. 164-175.

[19] Deng, W., Hu., F., Blaszczyński, J., Slowiński, R., Szeląg, M., Wang, G., 2013. A novel method for elimination of inconsistencies in ordinal classification with monotonicity constraints. Fundamenta Informaticae 126 (4), 377-395.

[20] Dias, L., Mousseau, V., Figueira, J., Clímaco, J., 2002. An aggregation/disaggregation approach to obtain robust conclusions with ELECTRE TRI. European Journal of Operational Research 138, 332-348.

[21] Doumpos, M., Zopounidis, C., 2011. Preference disaggregation and statistical learning for multicriteria decision support: A review. European Journal of Operational Research 209 (3), 203-214.

[22] Dubois, C., Bergeron, O., Potvin, A., Adolphe, L., 2012. Adapting cities to climate change: heat and urban form. In: The 8th International Conference on Urban Climates (ICUC 8). UCD, Dublin, Ireland.

[23] Figueira, J., Mousseau, V., Roy, B., 2005. Electre methods. In: Figueira, J., Greco, S., Ehrgott, M. (Eds.), Multiple criteria decision analysis: State of the art surveys. Springer-Verlag, New York, pp. 133-162. 
[Post print version, please cite as] Chakhar Salem, Ishizaka Alessio, Labib Ashraf, Saad Inès, Dominance-based Rough Set Approach for Group Decisions, European Journal of Operational Research, advance online publications, doi:10.1016/j.ejor.2015.10.060

[24] Greco, S., Kadziński, M., Mousseau, V., Slowiński, R., 2012. Robust ordinal regression for multiple criteria group decision: UTA ${ }^{\mathrm{GMS}}$-GROUP and UTADIS ${ }^{\mathrm{GMS}}$ _GROUP. Decision Support Systems 52 (3), 549-561.

[25] Greco, S., Matarazzo, B., Slowiński, 2008. Dominance-based rough set approach to interactive multiobjective optimization. In: Branke, J., Deb, K., Miettinen, K., Slowiński, R. (Eds.), Multiobjective Optimization. Vol. 5252 of Lecture Notes in Computer Science. Springer Berlin Heidelberg, pp. 121-155.

[26] Greco, S., Matarazzo, B., Slowiński, R., 1999. Advances in Multiple Criteria Decision Making. Kluwer Academic Publishers, Dordrecht, Boston, Ch. The use of rough sets and fuzzy sets in MCDM, pp. 14.1-14.59.

[27] Greco, S., Matarazzo, B., Slowiński, R., 2001. Rough sets theory for multicriteria decision analysis. European Journal of Operational Research 129 (1), 1-47.

[28] Greco, S., Matarazzo, B., Slowiński, R., 2002. Rough approximation by dominance relations. International Journal of Intelligent Systems 17 (2), 153-171.

[29] Greco, S., Matarazzo, B., Slowiński, R., 2006. Dominance-based rough set approach to decision involving multiple decision makers. In: Greco, S., Hata, Y., Hirano, S., Inuiguchi, M., Miyamoto, S., Nguyen, H., Slowiński, R. (Eds.), Proceedings of the 5th International Conference Rough Sets and Current Trends in Computing (RSCTC 2006), Kobe, Japan, November 6-8. Vol. 4259 of Lecture Notes in Artificial Intelligence. Springer-Verlag, Berlin Heidelberg, pp. 306-317.

[30] Greco, S., Matarazzo, B., Slowiński, R., 2007. Customer satisfaction analysis based on rough set approach. Zeitschrift fur Betriebswirtschaft 77 (3), 325-339.

[31] Greco, S., Matarazzo, B., Slowinski, R., Stefanowski, J., 2001. An algorithm for induction of decision rules consistent with the dominance principle. In: Ziarko, W., Yao, Y. (Eds.), Rough Sets and Current Trends in Computing. Vol. 2005 of Lecture Notes in Computer Science. Springer Berlin Heidelberg, pp. 304-313.

[32] Greco, S., Matarazzo, B., Slowiński, R., Stefanowski, J., 2001. Variable consistency model of dominancebased rough sets approach. In: Ziarko, W., Yao, Y. (Eds.), Rough Sets and Current Trends in Computing. Vol. 2005 of Lecture Notes in Computer Science. Springer Berlin / Heidelberg, pp. 170-181.

[33] Guay, G., Joerin, F., Chakhar, S., Villeneuve, P., Lavoie, C., 21-24 November 2011. FLORAIDE, a new decision-making tool for weed species. In: The 2nd World Conference on Biological Invasions and Ecosystem Functioning Group. Mar del Plata, Argentina.

[34] Herowati, E., Ciptomulyono, U., Parung, J., 2014. Expertise-based experts importance weights in adverse judgment. ARPN Journal of Engineering and Applied Sciences 9 (9), 1428-1435.

[35] Ishizaka, A., Labib, A., 2011. Selection of new production facilities with the group analytic hierarchy process ordering method. Expert Systems With Applications 38 (6), 7317-7325.

[36] Ishizaka, A., Nemery, P., 2013. Multi-criteria Decision Analysis: Methods and Software. Wiley.

[37] Ishizaka, A., Nemery, P., 2013. A multi-criteria group decision framework for partner grouping when sharing facilitiese. Group Decision and Negotiation 22 (4), 773-799.

[38] Jabeur, K., Martel, J.-M., 2007. An ordinal sorting method for group decision-making. European Journal of Operational Research 180, 1272-1289.

[39] Kadziński, M., Greco, S., Slowiński, R., 2014. Robust ordinal regression for dominance-based rough set approach to multiple criteria sorting. Information Sciences 283, 211-228.

[40] Kadziński, M., Slowiński, R., 2013. DIS-CARD: a new method of multiple criteria sorting to classes with desired cardinality. Journal of Global Optimization 56 (3), 1143-1166.

[41] Kadziński, M., Slowiński, R., Greco, S., 2015. Multiple criteria ranking and choice with all compatible minimal cover sets of decision rules. Knowledge-Based Systems 89, $569-583$. 
[Post print version, please cite as] Chakhar Salem, Ishizaka Alessio, Labib Ashraf, Saad Inès, Dominance-based Rough Set Approach for Group Decisions, European Journal of Operational Research, advance online publications, doi:10.1016/j.ejor.2015.10.060

[42] Labib, A., Read, M., Gladstone-Millar, C., Tonge, R., Smith, D., 2014. Formulation of higher education institutional strategy using operational research approaches. Studies in Higher Education 39 (5), 885-904.

[43] Legay, C., Cloutier, G., Chakhar, S., Joerin, F., Rodriguez, M., 2015. Estimation of urban water supply issues at the local scale: a participatory approach. Climatic Change $130(4), 491-503$.

[44] Leyva-López, J., Fernández-González, E., 2003. A new method for group decision support based on ELECTRE III methodology. European Journal of Operational Research 148 (1), 14-27.

[45] Li, S., Li, T., 2015. Incremental update of approximations in dominance-based rough sets approach under the variation of attribute values. Information Sciences 294, $348-361$

[46] Liou, J., Tang, C.-H., Yeh, W.-C., Tsai, C.-Y., 2011. A decision rules approach for improvement of airport service quality. Expert Systems with Applications 38 (11), 13723-13730.

[47] Liu, J., Liao, X., Yang, J., 2015. A group decision-making approach based on evidential reasoning for multiple criteria sorting problem with uncertainty. European Journal of Operational Research 246 (3), 858 - 873.

[48] Lolli, F., Ishizaka, A., Gamberini, R., Rimini, B., Messori, M., 2015. FlowSort-GDSS - a novel group multicriteria decision support system for sorting problems with application to FMEA. Expert Systems with Applications 42 (17-18), 6342-6349.

[49] Mercat-Rommens, C., Chakhar, S., Chojnacki, E., Mousseau, V., 2015. Coupling GIS and multi-criteria modeling to support post-accident nuclear risk evaluation. In: Bisdorff, R., Dias, L., Meyer, P., Mousseau, V., Pirlot, M. (Eds.), Evaluation and Decision Models with Multiple Criteria. International Handbooks on Information Systems. Springer Berlin Heidelberg, pp. 401-428.

[50] Paolotti, L., Greco, S., Boggia, A., 2015. Multiobjective strategies for farms, using the dominance-based rough set approach. Aestimum (65), 95-115.

[51] Pawlak, Z., 1991. Rough set. Theoretical aspects of reasoning about data. Kluwer Academic Publishers, Dordrecht.

[52] Pawlak, Z., 2005. Some remarks on conflict analysis. European Journal of Operational Research 166 (3), 649-654.

[53] Saad, I., Chakhar, S., 2009. A decision support for identifying crucial knowledge requiring capitalizing operation. European Journal of Operational Research 195 (3), 889-904.

[54] Saad, I., Chakhar, S., 2012. Multi-criteria methodology based on majority principle for collective identification of firm's valuable knowledge. Knowledge Management Research \& Practice 10 (4), 380-391.

[55] Shanteau, J., Weiss, D., Thomas, R., Pounds, J., 2002. Performance-based assessment of expertise: How to decide if someone is an expert or not. European Journal of Operational Research 136 (2), 253-263.

[56] Slowiński, R., Greco, S., Matarazzo, B., 2002. Rough set analysis of preference-ordered data. In: Alpigini, J., Peters, J., Skowron, A., Zhong, N. (Eds.), Rough Sets and Current Trends in Computing. Vol. 2475 of Lecture Notes in Artificial Intelligence. Springer Berlin Heidelberg, pp. 44-59.

[57] Slowiński, R., Kadziński, M., Greco, S., 2014. Robust ordinal regression for dominance-based rough set approach under uncertainty. In: Kryszkiewicz, M., Cornelis, C., Ciucci, D., Medina-Moreno, J., Motoda, H., Raś, Z. (Eds.), Rough Sets and Intelligent Systems Paradigms. Vol. 8537 of Lecture Notes in Computer Science. Springer International Publishing, pp. 77-87.

[58] Slowiński, R., Stefanowski, J., Greco, S., Matarazzo, B., 2000. Rough sets based processing of inconsistent information in decision analysis. Control and Cybernetics 29 (1), 379-404.

[59] StatsToDo Software, 2015. StatsToDo Trading Pty Ltd. https://www.statstodo.com/ StatsToDoIndex.php. 
[60] Sun, B., Ma, W., 2015. Rough approximation of a preference relation by multi-decision dominance for a multi-agent conflict analysis problem. Information Sciences 315, 39-53.

[61] Susmaga, R., Slowiński, 2015. Generation of rough sets reducts and constructs based on inter-class and intraclass information. Fuzzy Sets and Systems 274, 124 - 142, fuzzy Modeling for Optimisation and Decision Support.

[62] Waegeman, W., De Baets, B., Boullart, L., 2009. Kernel-based learning methods for preference aggregation. 4OR 7 (2), 169-189.

[63] Wang, H., Zhou, M., She, K., 2015. Induction of ordinal classification rules from decision tables with unknown monotonicity. European Journal of Operational Research 242 (1), 172-181.

[64] Weiss, D., Shanteau, J., 2003. Empirical assessment of expertise. Human Factors 45 (1), 104-116.

[65] Wen-Jie, B., Xiao-Hong, C., 20-22 August 2007. An extended dominance-based rough set approach to group sorting decision making. In: International Conference on Management Science and Engineering (ICMSE 2007). IEEE, Harbin, China, pp. 339-344.

[66] Wessa, P., 2012. Free Statistics Software, Office for Research Development and Education, version 1.1.23-r7. http://www.wessa.net/.

[67] Yang, X., Qi, Y., Yu, D.-J., Yu, H., Yang, J., 2015. $\alpha$-dominance relation and rough sets in interval-valued information systems. Information Sciences 294, $334-347$.

[68] Yue, Z., 2011. A method for group decision-making based on determining weights of decision makers using TOPSIS. Applied Mathematical Modelling 35 (4), 1926-1936.

[69] Zhang, F., Ignatius, J., Lim, C., Goh, M., 2014. A two-stage dynamic group decision making method for processing ordinal information. Knowledge-Based Systems 70, 189-202.

[70] Zopounidis, C., Doumpos, M., 2002. Multicriteria classification and sorting methods: A literature review. European Journal of Operational Research 138 (2), 229-246. 


\section{AppendixA. Proofs}

AppendixA.1. Properties of concordance power $S(\cdot, \cdot)$

Property 1. $0 \leq S\left(x, C l_{t}^{\diamond}\right) \leq 1, \forall t$ and $\diamond \in\{\geq, \leq\}$.

Proof. Let $\diamond \in\{\geq, \leq\}$. $S\left(x, C l_{t}^{\diamond}\right)$ is minimal when $L\left(x, C l_{t}^{\diamond}\right)=\emptyset$, i.e., $\nexists i \in H$, such that $x \in \underline{P}\left(C l_{t, i}^{\diamond}\right)$. In this case and based on Equation (5), we have: $S_{i}\left(x, C l_{t}^{\diamond}\right)=0(i=1, \cdots, h)$. Then by Equation (4), we obtain: $S\left(x, C l_{t}^{\diamond}\right)=S_{1}\left(x, C l_{t}^{\diamond}\right)+\cdots+S_{h}\left(x, C l_{t}^{\diamond}\right)=0$. Consequently, $S\left(x, C l_{t}^{\diamond}\right) \geq 0 . S\left(x, C l_{t}^{\diamond}\right)$ is maximal when $L\left(x, C l_{t}^{\diamond}\right)=H$. In this case and based on Equation (5), we have: $S_{i}\left(x, C l_{t}^{\diamond}\right)=\pi_{i}\left(C l_{t}^{\diamond}\right)(i=1, \cdots, h)$. Then by Equation (4), we obtain: $S\left(x, C l_{t}^{\diamond}\right)=\pi_{1}\left(C l_{t}^{\diamond}\right)+\cdots+\pi_{h}\left(C l_{t}^{\diamond}\right)=1$. Consequently, $0\left(x, C l_{t}^{\diamond}\right) \leq 1$. Finally, we get: $0 \leq S\left(x, C l_{t}^{\diamond}\right) \leq 1$.

Property 2. $S\left(x, C l_{t}^{\leq}\right) \leq S\left(x, C l_{s}^{\leq}\right), \forall s \geq t$.

Proof. By definition of the downward union we have $C l_{\bar{t}}^{\leq} \subseteq C l_{s}^{\leq}, \forall s \geq t$. This leads to: $\underline{P}\left(C l_{t}^{\leq}\right) \subseteq \underline{P}\left(C l_{\bar{s}}^{\leq}\right)$, $\forall s \geq t$. This means that: $x \in \underline{P}\left(C l_{t}^{\leq}\right) \Rightarrow x \in \underline{P}\left(C l_{s}^{\leq}\right), \forall s \geq t$. Then $L\left(x, C l_{t}^{\leq}\right) \subseteq L\left(x, C l_{s}^{\leq}\right), \forall s \geq t$. Consequently, $S\left(x, C l_{t}^{\leq}\right) \leq S\left(x, C l_{s}^{\leq}\right), \forall s \geq t$.

Property 3. $S\left(x, C l_{t}^{\geq}\right) \geq S\left(x, C l_{\bar{s}}^{\geq}\right), \forall s \geq t$.

Proof. By definition of the upward union we have $C l_{\bar{s}}^{\geq} \subseteq C l_{t}^{\geq}, \forall s \geq t$. This leads to: $\underline{P}\left(C l_{\bar{s}}^{\geq}\right) \subseteq \underline{P}\left(C l_{t}^{\geq}\right), \forall s \geq t$. This means that: $x \in \underline{P}\left(C l_{t}^{\leq}\right) \Rightarrow x \in \underline{P}\left(C l_{s}^{\leq}\right), \forall s \geq t$. Then $L\left(x, C l_{\bar{s}}^{\geq}\right) \subseteq L\left(x, C l_{t}^{\geq}\right), \forall s \geq t$ Consequently, $S\left(x, C l_{t}^{\geq}\right) \geq S\left(x, C l_{s}^{\geq}\right), \forall s \geq t$.

AppendixA.2. Properties of discordance power $Z(\cdot, \cdot)$

Property 4. $0 \leq Z\left(x, C l_{t}^{\diamond}\right) \leq 1, \forall t$ and $\diamond \in\{\geq, \leq\}$.

Proof. Let $\diamond \in\{\geq, \leq\}$. Two extreme cases may be distinguished in the definition of $Z\left(x, C l_{t}^{\diamond}\right)$. The first case holds when: $\pi_{i}\left(C l_{t}^{\diamond}\right)>S\left(x, C l_{t}^{\diamond}\right) \wedge i \in B\left(x, C l_{t}^{\diamond}\right), \forall i \in H$. In this case and based on Equation (7), we have: $Z_{i}\left(x, C l_{t}^{\diamond}\right)=\frac{1-\pi_{i}\left(C l_{t}^{\diamond}\right)}{1-S\left(x, C l_{t}^{\diamond}\right)}(i=1, \cdots, h)$. By definition we have: $0 \leq \pi_{i}\left(C l_{t}^{\diamond}\right) \leq 1$ and by Property (1) we have $0 \leq S\left(x, C l_{t}^{\diamond}\right) \leq 1$, we conclude that: $0 \leq Z_{i}\left(x, C l_{t}^{\diamond}\right) \leq 1$. Consequently: $0 \leq Z\left(x, C l_{t}^{\diamond}\right) \leq 1$. The second case holds when $\pi_{i}\left(C l_{t}^{\diamond}\right) \leq S\left(x, C l_{t}^{\diamond}\right)$ and $i \notin B\left(x, C l_{t}^{\diamond}\right), \forall i \in H$. In this case we have by definition $Z_{i}\left(x, C l_{t}^{\diamond}\right)=1$, $\forall i \in H$. Consequently: $0 \leq Z\left(x, C l_{t}^{\diamond}\right) \leq 1$.

AppendixA.3. Properties of the credibility index $\sigma(\cdot, \cdot)$

Property 5. $\sigma\left(x, C l_{t}^{\leq}\right) \leq \sigma\left(x, C l_{t^{\prime}}^{\leq}\right), \forall t^{\prime} \geq t$.

Proof. By definition we have: $\sigma\left(x, C l_{s}^{\leq}\right)=S\left(x, C l_{s}^{\leq}\right) \cdot Z\left(x, C l_{s}^{\leq}\right)$. Based on Property (2), we have: $S\left(x, C l_{t}^{\leq}\right) \leq$ $S\left(x, C l_{t^{\prime}}^{\leq}\right), \forall t^{\prime} \geq t$. This means that: $1-S\left(x, C l_{t}^{\leq}\right) \geq 1-S\left(x, C l_{t^{\prime}}^{\leq}\right), \forall t^{\prime} \geq t$. Then, since $0 \leq \pi_{k}\left(C l_{s}\right) \leq 1$, $\forall k$, we obtain $\frac{1-\pi_{k}\left(C l_{t}\right)}{1-S\left(x, C l_{t}^{\leq}\right)} \leq \frac{1-\pi_{k}\left(C l_{t^{\prime}}\right)}{1-S\left(x, C l_{t^{\prime}}^{\leq}\right)}, \forall k, \forall t^{\prime} \geq t$. This means that $Z_{k}\left(x, C l_{t}^{\leq}\right) \leq Z_{k}\left(x, C l_{t^{\prime}}^{\leq}\right), \forall k, \forall t^{\prime} \geq t$. Consequently, we have: $Z\left(x, C l_{t}^{\leq}\right) \leq Z\left(x, C l_{t^{\prime}}^{\leq}\right)$. This leads to: $S\left(x, C l_{t}^{\leq}\right) \cdot Z\left(x, C l_{t}^{\leq}\right) \leq S\left(x, C l_{t^{\prime}}^{\leq}\right) \cdot Z\left(x, C l_{t^{\prime}}^{\leq}\right)$. Finally, we obtain: $\sigma\left(x, C l_{t}^{\leq}\right) \leq \sigma\left(x, C l_{t^{\prime}}^{\leq}\right)$.

Property 6. $\sigma\left(x, C l_{t}^{\geq}\right) \geq \sigma\left(x, C l_{t^{\prime}}^{\geq}\right), \forall t^{\prime} \geq t$.

Proof. By definition we have: $\sigma\left(x, C l_{s}^{\geq}\right)=S\left(x, C l_{s}^{\geq}\right) \cdot Z\left(x, C l_{s}^{\geq}\right)$. Based on Property (3), we have: $S\left(x, C l_{t}^{\geq}\right) \geq$ $S\left(x, C l_{t^{\prime}}^{\geq}\right), \forall t^{\prime} \geq t$. This means that: $1-S\left(x, C l_{t}^{\geq}\right) \leq 1-S\left(x, C l_{t^{\prime}}^{\geq}\right), \forall t^{\prime} \geq t$. Then, since $0 \leq \pi_{k}\left(C l_{s}\right) \leq 1$, $\forall k$, we obtain $\frac{1-\pi_{k}\left(C l_{t}\right)}{1-S\left(x, C l_{t}^{\leq}\right)} \geq \frac{1-\pi_{k}\left(C l_{t^{\prime}}\right)}{1-S\left(x, C l_{t^{\prime}}^{\leq}\right)}, \forall k, \forall t^{\prime} \geq t$. This means that $Z_{k}\left(x, C l_{t}^{\leq}\right) \geq Z_{k}\left(x, C l_{t^{\prime}}^{\leq}\right), \forall k, \forall t^{\prime} \geq t$. Consequently, we have: $Z\left(x, C l_{t}^{\leq}\right) \geq Z\left(x, C l_{t^{\prime}}^{\leq}\right)$. This leads to: $S\left(x, C l_{t}^{\leq}\right) \cdot Z\left(x, C l_{t}^{\leq}\right) \geq S\left(x, C l_{t^{\prime}}^{\leq}\right) \cdot Z\left(x, C l_{t^{\prime}}^{\leq}\right)$. Finally, we obtain: $\sigma\left(x, C l_{t}^{\leq}\right) \geq \sigma\left(x, C l_{t^{\prime}}^{\leq}\right)$. 
AppendixA.4. Property of assignment intervals $I(x)$

Property 7. $C l_{0} \leq l(x) \leq C l_{n}$.

Proof. We have $l(x)=\operatorname{argmax}_{C l_{t}} N_{1}(x)$. By definition, we have $N_{1}(x)=\left\{C l_{t}: x \in C l_{t}^{\geq}\right\}$. Or the lower approximation of $C l_{t}^{\geq}$is defined for $t=1, \cdots, n$. Then $\operatorname{argmax}_{C l_{t}} N_{1}(x) \geq C l_{1}$. This leads to $l(x) \geq C l_{1}$ and then $l(x) \geq C l_{0}$.

Property 8. $C l_{0} \leq u(x) \leq C l_{n}$.

Proof. We have $u(x)=\operatorname{argmin}_{C l_{t}} N_{2}(x)$. By definition, we have $N_{2}(x)=\left\{C l_{t}: x \in C l_{t}^{\leq}\right\}$. Or the lower approximation of $C l_{t}^{\leq}$is defined for $t=0, \cdots, n-1$. Then $\operatorname{argmin}_{C l_{t}} N_{2}(x) \leq C l_{n-1}$. This leads to $u(x) \leq C l_{n-1}$ and then $u(x) \leq C l_{n}$.

Property 9. $l(x) \leq u(x), \forall x \in U$.

Proof. Case 1. $l(x)=C l_{0}$ and $u(x)=C l_{n}$. Then, $l(x) \leq u(x)$ is obvious. Case 2. $l(x)=\operatorname{argmax}_{C l_{t}} N_{1}(x)$ and $u(x)=C l_{n}$. Then, $l(x) \leq u(x)$ is obvious since $\operatorname{argmax}_{C l_{t}} N_{1}(x) \leq C l_{n}$. Case 3. $l(x)=C l_{0}$ and $u(x)=$ $\operatorname{argmin}_{C l_{t}} N_{2}(x)$. Then, $l(x) \leq u(x)$ is obvious since $\operatorname{argmin}_{C l_{t}} N_{2}(x) \geq C l_{0}$. Case $4 . l(x)=\operatorname{argmax}_{C l_{t}} N_{1}(x)$ and $u(x)=\operatorname{argmin}_{C l_{t}} N_{2}(x)$. Let $t, t_{1}, t_{2} \in T$ and $\lambda \in[0,1]$. By definition we have: $N_{1}(x)=\left\{C l_{t}: \sigma\left(x, C l_{t}^{\geq}\right) \geq \lambda\right\}$ and $N_{2}(x)=\left\{C l_{t}: \sigma\left(x, C l_{t}^{\leq}\right) \geq \lambda\right\}$. Assume that $l(x)=\operatorname{argmax}_{C l_{t}} N_{1}(x)=C l_{t_{1}}$ and $u(x)=\operatorname{argmin}_{C l_{t}} N_{2}(x)=$ $C l_{t_{2}}$. If $t_{1} \leq t_{2}$, then $l(x) \leq u(x)$ holds. Let now assume that $t_{2}<t_{1}$. Let now show by contradiction that this situation (i.e., $t_{2}<t_{1}$ ) cannot hold.

- Since $\sigma\left(x, C l_{t_{2}}^{\leq}\right) \geq \lambda$ leads to $(i) x \in \underline{P}\left(C l_{t_{2}}^{\leq}\right)$and (ii) $x \notin \underline{P}\left(C l_{t_{2}+1}^{\geq}\right)$. Or by $\sigma\left(x, C l_{t_{1}}^{\geq}\right) \leq \lambda$ we have $x \in \underline{P}\left(C l_{t_{1}}^{\geq}\right)$which contradicts (ii) since $\underline{P}\left(C l_{t_{1}}^{\geq}\right) \subseteq \underline{P}\left(C l_{t_{2}+1}^{\geq}\right)$(because we assumed that $t_{2}<t_{1}$ which means that $t_{2}+1 \leq t_{1}$ ).

- Similarly, $\sigma\left(x, C l_{t_{1}}^{\geq}\right) \geq \lambda$ leads to (iii) $x \in \underline{P}\left(C l_{t_{1}}^{\geq}\right)$and (iv) $x \notin \underline{P}\left(C l_{t_{1}-1}^{\leq}\right)$. Or by $\sigma\left(x, C l_{t_{2}}^{\leq}\right) \geq \lambda$ we have $x \in \underline{P}\left(C l_{t_{2}}^{\leq}\right)$which contradicts (iv) since $\underline{P}\left(C l_{t_{1}-1}^{\leq}\right) \subseteq \underline{P}\left(C l_{t_{2}}^{\leq}\right)$(because we assumed that $t_{2}<t_{1}$ which means that $t_{1}-1 \geq t_{2}$ ).

This means that the situation $t_{2}<t_{1}$ in Case 4 cannot hold. Finally, we conclude that $l(x) \leq u(x), \forall x \in U$. 
[Post print version, please cite as] Chakhar Salem, Ishizaka Alessio, Labib Ashraf, Saad Inès, Dominance-based Rough Set Approach for Group Decisions, European Journal of Operational Research, advance online publications, doi:10.1016/j.ejor.2015.10.060

\section{AppendixB. Computing details}

\section{AppendixB.1. Quality and accuracy on individuals classifications}

The quality of the approximations and the accuracy of classes approximations are summarized in Table B.1.

Table B.1: Quality, accuracy of approximations

\begin{tabular}{|c|c|c|c|c|c|c|c|c|c|c|c|c|c|c|c|c|c|c|c|c|c|c|c|c|c|c|c|c|c|c|c|c|c|}
\hline \multirow{2}{*}{$\begin{array}{c}\text { Rating } \\
\text { agency }(k)\end{array}$} & \multirow[t]{2}{*}{$\gamma_{k}$} & \multicolumn{16}{|c|}{$\alpha_{k}\left(C l_{\bar{t}}^{\geq}\right)$} & \multicolumn{16}{|c|}{$\alpha_{k}\left(C l_{t}^{\leq}\right)$} \\
\hline & & $t=2$ & $t=3$ & $t=4$ & $t=5$ & $t=6$ & $t=7$ & $t=8$ & $t=9$ & $t=10$ & $t=11$ & $t=12$ & $t=13$ & $t=14$ & $4 t=15$ & $t=16$ & $t=17$ & $t=1$ & $t=2$ & $t=3$ & $t=4$ & $t=5$ & $t=6$ & $t=7$ & $t=8$ & $t=9$ & $t=10$ & $t=11$ & $t=12$ & $t=13$ & $t=14$ & $t=15$ & $t=16$ \\
\hline Fitch (1) & 0.89 & 1 & 1 & 1 & 1 & 1 & 1 & 0.88 & 0.91 & 0.91 & 0.89 & 1 & 1 & 1 & 1 & 1 & 1 & 0 & 1 & 1 & 1 & 1 & 1 & 0.57 & 0.75 & 0.75 & 0.82 & 1 & 1 & 1 & 1 & 1 & 1 \\
\hline Moody (2) & 0.89 & 1 & 1 & 1 & 1 & 1 & 0.88 & 0.88 & 0.91 & 0.89 & 1 & 1 & 1 & 1 & 1 & 1 & 1 & 1 & 1 & 1 & 1 & 1 & 0.40 & 0.57 & 0.75 & 0.82 & 1 & 1 & 1 & 1 & 1 & 1 & 1 \\
\hline S\&P (3) & 0.89 & 1 & 1 & 1 & 1 & 1 & 0.88 & 0.87 & 0.90 & 0.90 & 0.89 & 1 & 1 & 1 & 1 & 1 & 1 & 0 & 0 & 1 & 1 & 1 & 0.50 & 0.63 & 0.78 & 0.80 & 0.82 & 1 & 1 & 1 & 1 & 1 & 1 \\
\hline
\end{tabular}

AppendixB.2. Concordance powers

The Concordance powers are summarized in Table B.2.

Table B.2: Concordance powers

\begin{tabular}{|c|c|c|c|c|c|c|c|c|c|c|c|c|c|c|c|c|c|c|c|c|c|c|c|c|c|c|c|c|c|c|c|c|}
\hline \multirow[t]{2}{*}{$x_{i}$} & \multicolumn{16}{|c|}{$C l_{\bar{t}}^{\geq}$} & \multicolumn{16}{|c|}{$\mathrm{Cl}_{\frac{1}{t}}$} \\
\hline & $t=2$ & $t=3$ & $t=4$ & $t=5$ & $t=6$ & $t=7$ & $t=8$ & $t=9$ & $t=10$ & $t=11$ & $t=12$ & $t=13$ & $t=14$ & $t=15$ & $t=16$ & $t=17$ & $t=1$ & $t=2$ & $t=3$ & $t=4$ & $t=5$ & $t=6$ & $t=7$ & $t=8$ & $t=9$ & $t=10$ & $t=11$ & $t=12$ & $t=13$ & $t=14$ & $t=15$ & $t=16$ \\
\hline 1 & 1 & 1 & 1 & 1 & 1 & 1 & 1 & 1 & 1 & 1 & 1 & 1 & 1 & 1 & 1 & 0.67 & 0 & 0 & 0 & 0 & 0 & 0 & 0 & 0 & 0 & 0 & 0 & 0 & 0 & 0 & 0 & 0.33 \\
\hline 2 & 1 & 1 & 1 & 1 & 1 & 1 & 1 & 1 & 1 & 1 & 1 & 1 & 1 & 0.67 & 0 & 0 & 0 & 0 & 0 & 0 & 0 & 0 & 0 & 0 & 0 & 0 & 0 & 0 & 0 & 0.33 & 1 & 1 \\
\hline 3 & 1 & 1 & 1 & 1 & 1 & 1 & 0.67 & 0.34 & 0 & 0 & 0 & 0 & 0 & 0 & 0 & 0 & 0 & 0 & 0 & 0 & 0 & 0 & 0.36 & 0.67 & 1 & 1 & 1 & 1 & 1 & 1 & 1 & 1 \\
\hline 4 & 1 & 1 & 1 & 1 & 1 & 0.64 & 0.34 & 0 & 0 & 0 & 0 & 0 & 0 & 0 & 0 & 0 & 0 & 0 & 0 & 0 & 0 & 0 & 0.68 & 1 & 1 & 1 & 1 & 1 & 1 & 1 & 1 & 1 \\
\hline 5 & 1 & 0.33 & 0.33 & 0 & 0 & 0 & 0 & 0 & 0 & 0 & 0 & 0 & 0 & 0 & 0 & 0 & 0 & 0.66 & 0.67 & 1 & 1 & 1 & 1 & 1 & 1 & 1 & 1 & 1 & 1 & 1 & 1 & 1 \\
\hline 6 & 1 & 1 & 1 & 1 & 1 & 1 & 1 & 1 & 1 & 1 & 1 & 1 & 0.33 & 0 & 0 & 0 & 0 & 0 & 0 & 0 & 0 & 0 & 0 & 0 & 0 & 0 & 0 & 0 & 0.33 & 0.67 & 0.67 & 0.67 \\
\hline 7 & 1 & 1 & 1 & 1 & 1 & 1 & 1 & 1 & 1 & 1 & 1 & 1 & 1 & 1 & 1 & 1 & 0 & 0 & 0 & 0 & 0 & 0 & 0 & 0 & 0 & 0 & 0 & 0 & 0 & 0 & 0 & 0 \\
\hline 8 & 1 & 1 & 1 & 1 & 1 & 1 & 1 & 1 & 1 & 1 & 1 & 1 & 0.33 & 0 & 0 & 0 & 0 & 0 & 0 & 0 & 0 & 0 & 0 & 0 & 0 & 0 & 0 & 0 & 0.67 & 1 & 1 & 1 \\
\hline 9 & 1 & 1 & 1 & 1 & 1 & 1 & 1 & 1 & 1 & 1 & 1 & 1 & 1 & 1 & 1 & 0.67 & 0 & 0 & 0 & 0 & 0 & 0 & 0 & 0 & 0 & 0 & 0 & 0 & 0 & 0 & 0 & 0.33 \\
\hline 10 & 1 & 1 & 1 & 1 & 1 & 1 & 1 & 1 & 1 & 1 & 1 & 1 & 1 & 1 & 0.33 & 0 & 0 & 0 & 0 & 0 & 0 & 0 & 0 & 0 & 0 & 0 & 0 & 0 & 0 & 0 & 0.67 & 1 \\
\hline 11 & 1 & 1 & 1 & 1 & 1 & 1 & 1 & 1 & 1 & 1 & 1 & 1 & 1 & 1 & 1 & 1 & 0 & 0 & 0 & 0 & 0 & 0 & 0 & 0 & 0 & 0 & 0 & 0 & 0 & 0 & 0 & 0 \\
\hline 12 & 0.67 & 0.67 & 0 & 0 & 0 & 0 & 0 & 0 & 0 & 0 & 0 & 0 & 0 & 0 & 0 & 0 & 0.33 & 0.33 & 1 & 1 & 1 & 1 & 1 & 1 & 1 & 1 & 1 & 1 & 1 & 1 & 1 & 1 \\
\hline 13 & 1 & 1 & 1 & 1 & 1 & 0.68 & 0 & 0 & 0 & 0 & 0 & 0 & 0 & 0 & 0 & 0 & 0 & 0 & 0 & 0 & 0 & 0.27 & 1 & 1 & 1 & 1 & 1 & 1 & 1 & 1 & 1 & 1 \\
\hline 14 & 1 & 1 & 1 & 1 & 1 & 1 & 1 & 1 & 1 & 0.64 & 0.33 & 0.33 & 0.33 & 0 & 0 & 0 & 0 & 0 & 0 & 0 & 0 & 0 & 0 & 0 & 0 & 0.38 & 0.67 & 1 & 1 & 1 & 1 & 1 \\
\hline 15 & 1 & 1 & 1 & 1 & 1 & 1 & 1 & 0.67 & 0.34 & 0 & 0 & 0 & 0 & 0 & 0 & 0 & 0 & 0 & 0 & 0 & 0 & 0 & 0 & 0.34 & 0.68 & 1 & 1 & 1 & 1 & 1 & 1 & 1 \\
\hline 16 & 1 & 1 & 1 & 1 & 1 & 0.36 & 0 & 0 & 0 & 0 & 0 & 0 & 0 & 0 & 0 & 0 & 0 & 0 & 0 & 0 & 0 & 0 & 0 & 0 & 0 & 0.38 & 1 & 1 & 1 & 1 & 1 & 1 \\
\hline 17 & 1 & 1 & 1 & 1 & 1 & 1 & 1 & 1 & 1 & 0.64 & 0 & 0 & 0 & 0 & 0 & 0 & 0 & 0 & 0 & 0 & 0 & 0 & 0 & 0 & 0 & 0.38 & 1 & 1 & 1 & 1 & 1 & 1 \\
\hline 18 & 1 & 1 & 1 & 1 & 1 & 1 & 1 & 1 & 1 & 1 & 1 & 1 & 1 & 1 & 1 & 1 & 0 & 0 & 0 & 0 & 0 & 0 & 0 & 0 & 0 & 0 & 0 & 0 & 0 & 0 & 0 & 0 \\
\hline 19 & 1 & 1 & 1 & 1 & 1 & 1 & 1 & 1 & 1 & 1 & 0.33 & 0.33 & 0 & 0 & 0 & 0 & 0 & 0 & 0 & 0 & 0 & 0 & 0 & 0 & 0 & 0.311 & 0.67 & 0.67 & 1 & 1 & 1 & 1 \\
\hline 20 & 1 & 1 & 1 & 1 & 1 & 1 & 1 & 1 & 1 & 1 & 1 & 1 & 1 & 1 & 1 & 0.67 & 0 & 0 & 0 & 0 & 0 & 0 & 0 & 0 & 0 & 0 & 0 & 0 & 0 & 0 & 0 & 0 \\
\hline 21 & 1 & 1 & 1 & 1 & 1 & 1 & 1 & 1 & 1 & 1 & 1 & 1 & 1 & 1 & 1 & 1 & 0 & 0 & 0 & 0 & 0 & 0 & 0 & 0 & 0 & 0 & 0 & 0 & 0 & 0 & 0 & 0 \\
\hline 22 & 1 & 1 & 1 & 1 & 1 & 1 & 1 & 1 & 1 & 1 & 0.33 & 0 & 0 & 0 & 0 & 0 & 0 & 0 & 0 & 0 & 0 & 0 & 0 & 0 & 0 & 0 & 0.67 & 1 & 1 & 1 & 1 & 1 \\
\hline 23 & 1 & 1 & 1 & 1 & 1 & 0.36 & 0 & 0 & 0 & 0 & 0 & 0 & 0 & 0 & 0 & 0 & 0 & 0 & 0 & 0 & 0 & 0 & 0 & 0 & 0 & 0.38 & 1 & 1 & 1 & 1 & 1 & 1 \\
\hline 24 & 1 & 1 & 1 & 1 & 1 & 0.36 & 0.34 & 0 & 0 & 0 & 0 & 0 & 0 & 0 & 0 & 0 & 0 & 0 & 0 & 0 & 0 & 0 & 0 & 1 & 1 & 1 & 1 & 1 & 1 & 1 & 1 & 1 \\
\hline 25 & 1 & 1 & 1 & 1 & 1 & 1 & 0.67 & 0.67 & 0.67 & 0.32 & 0 & 0 & 0 & 0 & 0 & 0 & 0 & 0 & 0 & 0 & 0 & 0 & 0.32 & 0.34 & 0.35 & 0.69 & 1 & 1 & 1 & 1 & 1 & 1 \\
\hline 26 & 1 & 1 & 1 & 1 & 1 & 1 & 1 & 1 & 0.34 & 0 & 0 & 0 & 0 & 0 & 0 & 0 & 0 & 0 & 0 & 0 & 0 & 0 & 0 & 0 & 0.69 & 1 & 1 & 1 & 1 & 1 & 1 & 1 \\
\hline 27 & 1 & 1 & 1 & 1 & 1 & 1 & 1 & 1 & 1 & 1 & 1 & 1 & 1 & 1 & 1 & 1 & 0 & 0 & 0 & 0 & 0 & 0 & 0 & 0 & 0 & 0 & 0 & 0 & 0 & 0 & 0 & 0 \\
\hline 28 & 1 & 1 & 1 & 1 & 1 & 1 & 1 & 1 & 1 & 1 & 1 & 1 & 1 & 1 & 1 & 0.33 & 0 & 0 & 0 & 0 & 0 & 0 & 0 & 0 & 0 & 0 & 0 & 0 & 0 & 0 & 0 & 0.67 \\
\hline
\end{tabular}

AppendixB.3. Discordance powers

The Discordance powers are summarized in Table B.3.

Table B.3: Discordance powers

\begin{tabular}{|c|c|c|c|c|c|c|c|c|c|c|c|c|c|c|c|c|c|c|c|c|c|c|c|c|c|c|c|c|c|c|c|c|}
\hline \multirow{2}{*}{$x_{i}$} & \multicolumn{16}{|c|}{$\mathrm{Cl}_{ \pm}^{\geq}$} & \multicolumn{16}{|c|}{$\mathrm{Cl}^{\leq} \leq$} \\
\hline & $t=2$ & $t=3$ & $t=4$ & $t=5$ & $t=6$ & $t=7$ & $t=8$ & $t=9$ & $t=10$ & $t=11$ & $t=12$ & $t=13$ & $t=14$ & $t=15$ & $t=16$ & $t=17$ & $t=1$ & $t=2$ & $t=3$ & $t=4$ & $t=5$ & $t=6$ & $t=7$ & $t=8$ & $t=9$ & $t=10$ & $t=11$ & $t=12$ & $t=13$ & $t=14$ & $t=15$ & $t=16$ \\
\hline 1 & 1 & 1 & 1 & 1 & 1 & 1 & 1 & 1 & 1 & 1 & 1 & 1 & 1 & 1 & 1 & 1 & 1 & 1 & 1 & 1 & 1 & 1 & 1 & 1 & 1 & 1 & 1 & 1 & 1 & 1 & 1 & 1 \\
\hline 2 & 1 & 1 & 1 & 1 & 1 & 1 & 1 & 1 & 1 & 1 & 1 & 1 & 1 & 1 & 1 & 1 & 1 & 1 & 1 & 1 & 1 & 1 & 1 & 1 & 1 & 1 & 1 & 1 & 1 & 1 & 1 & 1 \\
\hline 3 & 1 & 1 & 1 & 1 & 1 & 1 & 1 & 1 & 1 & 1 & 1 & 1 & 1 & 1 & 1 & 1 & 1 & 1 & 1 & 1 & 1 & 1 & 1 & 1 & 1 & 1 & 1 & 1 & 1 & 1 & 1 & 1 \\
\hline 4 & 1 & 1 & 1 & 1 & 1 & 1 & 1 & 1 & 1 & 1 & 1 & 1 & 1 & 1 & 1 & 1 & 1 & 1 & 1 & 1 & 1 & 1 & 1 & 1 & 1 & 1 & 1 & 1 & 1 & 1 & 1 & 1 \\
\hline 5 & 1 & 1 & 1 & 1 & 1 & 1 & 1 & 1 & 1 & 1 & 1 & 1 & 1 & 1 & 1 & 1 & 1 & 1 & 1 & 1 & 1 & 1 & 1 & 1 & 1 & 1 & 1 & 1 & 1 & 1 & 1 & 1 \\
\hline 6 & 1 & 1 & 1 & 1 & 1 & 1 & 1 & 1 & 1 & 1 & 1 & 1 & 1 & 1 & 1 & 1 & 1 & 1 & 1 & 1 & 1 & 1 & 1 & 1 & 1 & 1 & 1 & 1 & 1 & 1 & 1 & 1 \\
\hline 7 & 1 & 1 & 1 & 1 & 1 & 1 & 1 & 1 & 1 & 1 & 1 & 1 & 1 & 1 & 1 & 1 & 1 & 1 & 1 & 1 & 1 & 1 & 1 & 1 & 1 & 1 & 1 & 1 & 1 & 1 & 1 & 1 \\
\hline 8 & 1 & 1 & 1 & 1 & 1 & 1 & 1 & 1 & 1 & 1 & 1 & 1 & 1 & 1 & 1 & 1 & 1 & 1 & 1 & 1 & 1 & 1 & 1 & 1 & 1 & 1 & 1 & 1 & 1 & 1 & 1 & 1 \\
\hline 9 & 1 & 1 & 1 & 1 & 1 & 1 & 1 & 1 & 1 & 1 & 1 & 1 & 1 & 1 & 1 & 1 & 1 & 1 & 1 & 1 & 1 & 1 & 1 & 1 & 1 & 1 & 1 & 1 & 1 & 1 & 1 & 1 \\
\hline 10 & 1 & 1 & 1 & 1 & 1 & 1 & 1 & 1 & 1 & 1 & 1 & 1 & 1 & 1 & 1 & 1 & 1 & 1 & 1 & 1 & 1 & 1 & 1 & 1 & 1 & 1 & 1 & 1 & 1 & 1 & 1 & 1 \\
\hline 11 & 1 & 1 & 1 & 1 & 1 & 1 & 1 & 1 & 1 & 1 & 1 & 1 & 1 & 1 & 1 & 1 & 1 & 1 & 1 & 1 & 1 & 1 & 1 & 1 & 1 & 1 & 1 & 1 & 1 & 1 & 1 & 1 \\
\hline 12 & 1 & 1 & 1 & 1 & 1 & 1 & 1 & 1 & 1 & 1 & 1 & 1 & 1 & 1 & 1 & 1 & 1 & 1 & 1 & 1 & 1 & 1 & 1 & 1 & 1 & 1 & 1 & 1 & 1 & 1 & 1 & 1 \\
\hline 13 & 1 & 1 & 1 & 1 & 1 & 1 & 1 & 1 & 1 & 1 & 1 & 1 & 1 & 1 & 1 & 1 & 1 & 1 & 1 & 1 & 1 & 1 & 1 & 1 & 1 & 1 & 1 & 1 & 1 & 1 & 1 & 1 \\
\hline 14 & 1 & 1 & 1 & 1 & 1 & 1 & 1 & 1 & 1 & 1 & 1 & 1 & 1 & 1 & 1 & 1 & 1 & 1 & 1 & 1 & 1 & 1 & 1 & 1 & 1 & 1 & 1 & 1 & 1 & 1 & 1 & 1 \\
\hline 15 & 1 & 1 & 1 & 1 & 1 & 1 & 1 & 1 & 1 & 1 & 1 & 1 & 1 & 1 & 1 & 1 & 1 & 1 & 1 & 1 & 1 & 1 & 1 & 1 & 1 & 1 & 1 & 1 & 1 & 1 & 1 & 1 \\
\hline 16 & 1 & 1 & 1 & 1 & 1 & 1 & 0.30 & 0.30 & 0.30 & 0.46 & 1 & 1 & 1 & 1 & 1 & 1 & 1 & 1 & 1 & 1 & 1 & 0.58 & 0.30 & 0.30 & 0.30 & 1 & 1 & 1 & 1 & 1 & 1 & 1 \\
\hline 17 & 1 & 1 & 1 & 1 & 1 & 1 & 1 & 1 & 1 & 1 & 1 & 1 & 1 & 1 & 1 & 1 & 1 & 1 & 1 & 1 & 1 & 1 & 1 & 1 & 1 & 1 & 1 & 1 & 1 & 1 & 1 & 1 \\
\hline 18 & 1 & 1 & 1 & 1 & 1 & 1 & 1 & 1 & 1 & 1 & 1 & 1 & 1 & 1 & 1 & 1 & 1 & 1 & 1 & 1 & 1 & 1 & 1 & 1 & 1 & 1 & 1 & 1 & 1 & 1 & 1 & 1 \\
\hline 19 & 1 & 1 & 1 & 1 & 1 & 1 & 1 & 1 & 1 & 1 & 1 & 1 & 1 & 1 & 1 & 1 & 1 & 1 & 1 & 1 & 1 & 1 & 1 & 1 & 1 & 1 & 1 & 1 & 1 & 1 & 1 & 1 \\
\hline 20 & 1 & 1 & 1 & 1 & 1 & 1 & 1 & 1 & 1 & 1 & 1 & 1 & 1 & 1 & 1 & 1 & 1 & 1 & 1 & 1 & 1 & 1 & 1 & 1 & 1 & 1 & 1 & 1 & 1 & 1 & 1 & 1 \\
\hline 21 & 1 & 1 & 1 & 1 & 1 & 1 & 1 & 1 & 1 & 1 & 1 & 1 & 1 & 1 & 1 & 1 & 1 & 1 & 1 & 1 & 1 & 1 & 1 & 1 & 1 & 1 & 1 & 1 & 1 & 1 & 1 & 1 \\
\hline 22 & 1 & 1 & 1 & 1 & 1 & 1 & 1 & 1 & 1 & 1 & 1 & 1 & 1 & 1 & 1 & 1 & 1 & 1 & 1 & 1 & 1 & 1 & 1 & 1 & 1 & 1 & 1 & 1 & 1 & 1 & 1 & 1 \\
\hline 23 & 1 & 1 & 1 & 1 & 1 & 1 & 0.30 & 0.30 & 0.30 & 0.46 & 1 & 1 & 1 & 1 & 1 & 1 & 1 & 1 & 1 & 1 & 1 & 0.58 & 0.30 & 0.30 & 0.30 & 1 & 1 & 1 & 1 & 1 & 1 & 1 \\
\hline 24 & 1 & 1 & 1 & 1 & 1 & 1 & 1 & 1 & 1 & 1 & 1 & 1 & 1 & 1 & 1 & 1 & 1 & 1 & 1 & 1 & 1 & 0.58 & 0.30 & 1 & 1 & 1 & 1 & 1 & 1 & 1 & 1 & 1 \\
\hline 25 & 1 & 1 & 1 & 1 & 1 & 1 & 1 & 1 & 1 & 1 & 1 & 1 & 1 & 1 & 1 & 1 & 1 & 1 & 1 & 1 & 1 & 1 & 1 & 1 & 1 & 1 & 1 & 1 & 1 & 1 & 1 & 1 \\
\hline 26 & 1 & 1 & 1 & 1 & 1 & 1 & 1 & 1 & 1 & 1 & 1 & 1 & 1 & 1 & 1 & 1 & 1 & 1 & 1 & 1 & 1 & 1 & 1 & 1 & 1 & 1 & 1 & 1 & 1 & 1 & 1 & 1 \\
\hline 27 & 1 & 1 & 1 & 1 & 1 & 1 & 1 & 1 & 1 & 1 & 1 & 1 & 1 & 1 & 1 & 1 & 1 & 1 & 1 & 1 & 1 & 1 & 1 & 1 & 1 & 1 & 1 & 1 & 1 & 1 & 1 & 1 \\
\hline 28 & 1 & 1 & 1 & 1 & 1 & 1 & 1 & 1 & 1 & 1 & 1 & 1 & 1 & 1 & 1 & 1 & 1 & 1 & 1 & 1 & 1 & 1 & 1 & 1 & 1 & 1 & 1 & 1 & 1 & 1 & 1 & 1 \\
\hline
\end{tabular}

AppendixB.4. Credibility indices

The Discordance powers are summarized in Table B.4. 
[Post print version, please cite as] Chakhar Salem, Ishizaka Alessio, Labib Ashraf, Saad Inès, Dominance-based Rough Set Approach for Group Decisions, European Journal of Operational Research, advance online publications, doi:10.1016/j.ejor.2015.10.060

Table B.4: Credibility indices

\begin{tabular}{|c|c|c|c|c|c|c|c|c|c|c|c|c|c|c|c|c|c|c|c|c|c|c|c|c|c|c|c|c|c|c|c|c|}
\hline \multirow[t]{2}{*}{$x_{i}$} & \multicolumn{16}{|c|}{$\mathrm{Cl}_{t}^{\geq}$} & \multicolumn{16}{|c|}{$\mathrm{Cl}_{t} \leq$} \\
\hline & $t=2$ & $t=3$ & $t=4$ & $t=5$ & $t=6$ & $t=7$ & $t=8$ & $t=9$ & $t=10$ & $t=11$ & $t=12$ & $t=13$ & $t=14$ & $t=15$ & $t=16$ & $t=17$ & $t=1$ & $t=2$ & $t=3$ & $t=4$ & $t=5$ & $t=6$ & $t=7$ & $t=8$ & $t=9$ & $t=10$ & $t=11$ & $t=12$ & $t=13$ & $t=14$ & $t=15$ & $t=16$ \\
\hline 1 & 1 & 1 & 1 & 1 & 1 & 1 & 1 & 1 & 1 & 1 & 1 & 1 & 1 & 1 & 1 & 0.67 & 0 & 0 & 0 & 0 & 0 & 0 & 0 & 0 & 0 & 0 & 0 & 0 & 0 & 0 & 0 & 0.33 \\
\hline 2 & 1 & 1 & 1 & 1 & 1 & 1 & 1 & 1 & 1 & 1 & 1 & 1 & 1 & 0.67 & 0 & 0 & 0 & 0 & 0 & 0 & 0 & 0 & 0 & 0 & 0 & 0 & 0 & 0 & 0 & 0.33 & 1 & 1 \\
\hline 3 & 1 & 1 & 1 & 1 & 1 & 1 & 0.67 & 0.34 & 0 & 0 & 0 & 0 & 0 & 0 & 0 & 0 & 0 & 0 & 0 & 0 & 0 & 0 & 0.36 & 0.67 & 1 & 1 & 1 & 1 & 1 & 1 & 1 & 1 \\
\hline 4 & 1 & 1 & 1 & 1 & 1 & 0.64 & 0.34 & 0 & 0 & 0 & 0 & 0 & 0 & 0 & 0 & 0 & 0 & 0 & 0 & 0 & 0 & 0 & 0.68 & 1 & 1 & 1 & 1 & 1 & 1 & 1 & 1 & 1 \\
\hline 5 & 1 & 0.33 & 0.33 & 0 & 0 & 0 & 0 & 0 & 0 & 0 & 0 & 0 & 0 & 0 & 0 & 0 & 0 & 0.66 & 0.67 & 1 & 1 & 1 & 1 & 1 & 1 & 1 & 1 & 1 & 1 & 1 & 1 & 1 \\
\hline 6 & 1 & 1 & 1 & 1 & 1 & 1 & 1 & 1 & 1 & 1 & 1 & 1 & 0.33 & 0 & 0 & 0 & 0 & 0 & 0 & 0 & 0 & 0 & 0 & 0 & 0 & 0 & 0 & 0 & 0.33 & 0.67 & 0.67 & 0.67 \\
\hline 7 & 1 & 1 & 1 & 1 & 1 & 1 & 1 & 1 & 1 & 1 & 1 & 1 & 1 & 1 & 1 & 1 & 0 & 0 & 0 & 0 & 0 & 0 & 0 & 0 & 0 & 0 & 0 & 0 & 0 & 0 & 0 & 0 \\
\hline 8 & 1 & 1 & 1 & 1 & 1 & 1 & 1 & 1 & 1 & 1 & 1 & 1 & 0.33 & 0 & 0 & 0 & 0 & 0 & 0 & 0 & 0 & 0 & 0 & 0 & 0 & 0 & 0 & 0 & 0.67 & 1 & 1 & 1 \\
\hline 9 & 1 & 1 & 1 & 1 & 1 & 1 & 1 & 1 & 1 & 1 & 1 & 1 & 1 & 1 & 1 & 0.67 & 0 & 0 & 0 & 0 & 0 & 0 & 0 & 0 & 0 & 0 & 0 & 0 & 0 & 0 & 0 & 0.33 \\
\hline 10 & 1 & 1 & 1 & 1 & 1 & 1 & 1 & 1 & 1 & 1 & 1 & 1 & 1 & 1 & 0.33 & 0 & 0 & 0 & 0 & 0 & 0 & 0 & 0 & 0 & 0 & 0 & 0 & 0 & 0 & 0 & 0.67 & 1 \\
\hline 11 & 1 & 1 & 1 & 1 & 1 & 1 & 1 & 1 & 1 & 1 & 1 & 1 & 1 & 1 & 1 & 1 & 0 & 0 & 0 & 0 & 0 & 0 & 0 & 0 & 0 & 0 & 0 & 0 & 0 & 0 & 0 & 0 \\
\hline 12 & 0.67 & 0.67 & 0 & 0 & 0 & 0 & 0 & 0 & 0 & 0 & 0 & 0 & 0 & 0 & 0 & 0 & 0.33 & 0.33 & 1 & 1 & 1 & 1 & 1 & 1 & 1 & 1 & 1 & 1 & 1 & 1 & 1 & 1 \\
\hline 13 & 1 & 1 & 1 & 1 & 1 & 0.68 & 0 & 0 & 0 & 0 & 0 & 0 & 0 & 0 & 0 & 0 & 0 & 0 & 0 & 0 & 0 & 0.26 & 1 & 1 & 1 & 1 & 1 & 1 & 1 & 1 & 1 & 1 \\
\hline 14 & 1 & 1 & 1 & 1 & 1 & 1 & 1 & 1 & 1 & 0.64 & 0.33 & 0.33 & 0.33 & 0 & 0 & 0 & 0 & 0 & 0 & 0 & 0 & 0 & 0 & 0 & 0 & 0.38 & 0.67 & 1 & 1 & 1 & 1 & 1 \\
\hline 15 & 1 & 1 & 1 & 1 & 1 & 1 & 1 & 0.67 & 0.34 & 0 & 0 & 0 & 0 & 0 & 0 & 0 & 0 & 0 & 0 & 0 & 0 & 0 & 0 & 0.34 & 0.69 & 1 & 1 & 1 & 1 & 1 & 1 & 1 \\
\hline 16 & 1 & 1 & 1 & 1 & 1 & 0.36 & 0 & 0 & 0 & 0 & 0 & 0 & 0 & 0 & 0 & 0 & 0 & 0 & 0 & 0 & 0 & 0 & 0 & 0 & 0 & 0.38 & 1 & 1 & 1 & 1 & 1 & 1 \\
\hline 17 & 1 & 1 & 1 & 1 & 1 & 1 & 1 & 1 & 1 & 0.64 & 0 & 0 & 0 & 0 & 0 & 0 & 0 & 0 & 0 & 0 & 0 & 0 & 0 & 0 & 0 & 0.38 & 1 & 1 & 1 & 1 & 1 & 1 \\
\hline 18 & 1 & 1 & 1 & 1 & 1 & 1 & 1 & 1 & 1 & 1 & 1 & 1 & 1 & 1 & 1 & 1 & 0 & 0 & 0 & 0 & 0 & 0 & 0 & 0 & 0 & 0 & 0 & 0 & 0 & 0 & 0 & 0 \\
\hline 19 & 1 & 1 & 1 & 1 & 1 & 1 & 1 & 1 & 1 & 1 & 0.33 & 0.33 & 0 & 0 & 0 & 0 & 0 & 0 & 0 & 0 & 0 & 0 & 0 & 0 & 0 & 0.31 & 0.67 & 0.67 & 1 & 1 & 1 & 1 \\
\hline 20 & 1 & 1 & 1 & 1 & 1 & 1 & 1 & 1 & 1 & 1 & 1 & 1 & 1 & 1 & 1 & 0.67 & 0 & 0 & 0 & 0 & 0 & 0 & 0 & 0 & 0 & 0 & 0 & 0 & 0 & 0 & 0 & 0 \\
\hline 21 & 1 & 1 & 1 & 1 & 1 & 1 & 1 & 1 & 1 & 1 & 1 & 1 & 1 & 1 & 1 & 1 & 0 & 0 & 0 & 0 & 0 & 0 & 0 & 0 & 0 & 0 & 0 & 0 & 0 & 0 & 0 & 0 \\
\hline 22 & 1 & 1 & 1 & 1 & 1 & 1 & 1 & 1 & 1 & 1 & 0.333 & 0 & 0 & 0 & 0 & 0 & 0 & 0 & 0 & 0 & 0 & 0 & 0 & 0 & 0 & 0 & 0.67 & 1 & 1 & 1 & 1 & 1 \\
\hline 23 & 1 & 1 & 1 & 1 & 1 & 0.36 & 0 & 0 & 0 & 0 & 0 & 0 & 0 & 0 & 0 & 0 & 0 & 0 & 0 & 0 & 0 & 0 & 0 & 0 & 0 & 0.38 & 1 & 1 & 1 & 1 & 1 & 1 \\
\hline 24 & 1 & 1 & 1 & 1 & 1 & 0.36 & 0.34 & 0 & 0 & 0 & 0 & 0 & 0 & 0 & 0 & 0 & 0 & 0 & 0 & 0 & 0 & 0 & 0 & 1 & 1 & 1 & 1 & 1 & 1 & 1 & 1 & 1 \\
\hline 25 & 1 & 1 & 1 & 1 & 1 & 1 & 0.67 & 0.67 & 0.67 & 0.32 & 0 & 0 & 0 & 0 & 0 & 0 & 0 & 0 & 0 & 0 & 0 & 0 & 0.32 & 0.33 & 0.34 & 0.69 & 1 & 1 & 1 & 1 & 1 & 1 \\
\hline 26 & 1 & 1 & 1 & 1 & 1 & 1 & 1 & 1 & 0.34 & 0 & 0 & 0 & 0 & 0 & 0 & 0 & 0 & 0 & 0 & 0 & 0 & 0 & 0 & 0 & 0.68 & 1 & 1 & 1 & 1 & 1 & 1 & 1 \\
\hline 27 & 1 & 1 & 1 & 1 & 1 & 1 & 1 & 1 & 1 & 1 & 1 & 1 & 1 & 1 & 1 & 1 & 0 & 0 & 0 & 0 & 0 & 0 & 0 & 0 & 0 & 0 & 0 & 0 & 0 & 0 & 0 & 0 \\
\hline 28 & 1 & 1 & 1 & 1 & 1 & 1 & 1 & 1 & 1 & 1 & 1 & 1 & 1 & 1 & 1 & 0.33 & 0 & 0 & 0 & 0 & 0 & 0 & 0 & 0 & 0 & 0 & 0 & 0 & 0 & 0 & 0 & 0.67 \\
\hline
\end{tabular}

\section{AppendixB.5. Assignment constraints}

The assignment constraints for $\lambda=0.75$ are given in Table B.5. These constraints are defined based on Equations (9) and (10). The symbol "x" means that the object $x$ is assigned to class $C l_{t}^{\diamond}$ with $\diamond \in\{\geq$, $\leq\}$, while symbol "." means that object $x$ is not assigned to this class.

Table B.5: Assignment constraints for $\lambda=0.75$

\begin{tabular}{|c|c|c|c|c|c|c|c|c|c|c|c|c|c|c|c|c|c|c|c|c|c|c|c|c|c|c|c|c|c|c|c|c|}
\hline \multirow{2}{*}{$x_{i}$} & \multicolumn{16}{|c|}{$\mathrm{Cl}_{t}^{\geq}$} & \multicolumn{16}{|c|}{$\mathrm{Cl}_{t} \leq$} \\
\hline & $t=2$ & $t=3$ & $t=4$ & $t=5$ & $t=6$ & $t=7$ & $t=8$ & $t=9$ & $t=10$ & $t=11$ & $t=12$ & $t=13$ & $t=14$ & $t=15$ & $t=16$ & $t=17$ & $t=1$ & $t=2$ & $t=3$ & $t=4$ & $t=5$ & $t=6$ & $t=7$ & $t=8$ & $t=9$ & $t=10$ & $t=11$ & $t=12$ & $t=13$ & $t=14$ & $t=15$ & $t=16$ \\
\hline 1 & $\mathrm{x}$ & $\mathrm{x}$ & $\mathrm{x}$ & $\begin{array}{ll}x \\
\end{array}$ & $\mathrm{x}$ & $\mathrm{x}$ & $\mathrm{x}$ & $\mathrm{x}$ & $\begin{array}{ll}x \\
\end{array}$ & $\begin{array}{ll}x \\
\end{array}$ & $\begin{array}{ll}x \\
\end{array}$ & $\begin{array}{ll}x \\
\end{array}$ & $\begin{array}{ll}x \\
\end{array}$ & $\begin{array}{ll}x \\
\end{array}$ & $\begin{array}{ll}x \\
\end{array}$ & - & - & - & - & - & - & - & - & - & - & - & - & 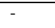 & - & - & - & - \\
\hline 2 & $\mathrm{x}$ & $\mathrm{x}$ & $\mathrm{x}$ & $\mathrm{x}$ & $\mathrm{x}$ & $\mathrm{x}$ & $\mathrm{x}$ & $\mathrm{x}$ & $\mathrm{x}$ & $\mathrm{x}$ & $\mathrm{x}$ & $\mathrm{x}$ & $\mathrm{x}$ & - & - & - & - & - & - & - & - & - & - & - & - & - & - & - & - & - & $x$ & $\mathrm{x}$ \\
\hline 3 & $\mathrm{x}$ & $\mathrm{x}$ & $\mathrm{x}$ & $\mathrm{x}$ & $\mathrm{x}$ & $\mathrm{x}$ & - & - & - & - & - & - & - & - & - & - & - & - & - & - & - & - & - & - & $\mathrm{x}$ & $x$ & $\mathrm{x}$ & $\mathrm{x}$ & $\mathrm{x}$ & $\mathrm{x}$ & $\mathrm{x}$ & $\mathrm{x}$ \\
\hline 4 & $\mathrm{x}$ & $\mathrm{x}$ & $\mathrm{x}$ & $\mathrm{x}$ & $x$ & - & - & - & - & - & - & - & - & - & - & - & - & - & - & - & - & - & - & $\mathrm{x}$ & $\mathrm{x}$ & $x$ & $\mathrm{x}$ & $\mathrm{x}$ & $\mathrm{x}$ & $\mathrm{x}$ & $\mathrm{x}$ & $\mathrm{x}$ \\
\hline 5 & $\mathrm{x}$ & - & - & - & - & - & - & - & - & - & - & - & - & - & - & - & - & - & - & $\mathrm{x}$ & $\mathrm{x}$ & $\mathrm{x}$ & $\mathrm{x}$ & $\mathrm{x}$ & $\mathrm{x}$ & $x$ & $\mathrm{x}$ & $\mathrm{x}$ & $\mathrm{x}$ & $\mathrm{x}$ & $\mathrm{x}$ & $\mathrm{x}$ \\
\hline 6 & $\mathrm{x}$ & $\mathrm{x}$ & $\mathrm{x}$ & $\mathrm{x}$ & $\mathrm{x}$ & $\mathrm{x}$ & $\mathrm{x}$ & $\mathrm{x}$ & $\mathrm{x}$ & $\mathrm{x}$ & $\mathrm{x}$ & $x$ & - & - & - & - & - & - & - & - & - & - & - & - & - & - & - & - & - & - & - & - \\
\hline 7 & $\mathrm{x}$ & $\mathrm{x}$ & $\mathrm{x}$ & $\mathrm{x}$ & $\mathrm{x}$ & $\mathrm{x}$ & $\mathrm{x}$ & $\mathrm{x}$ & $\mathrm{x}$ & $\mathrm{x}$ & $\mathrm{x}$ & $\mathrm{x}$ & $\mathrm{x}$ & $\mathrm{x}$ & $\mathrm{x}$ & $\mathrm{x}$ & - & - & - & - & - & - & - & - & - & - & - & - & - & - & - & - \\
\hline 8 & $\mathrm{x}$ & $\mathrm{x}$ & $\mathrm{x}$ & $\mathrm{x}$ & $x$ & $\mathrm{x}$ & $\mathrm{x}$ & $\mathrm{x}$ & $\mathrm{x}$ & $\mathrm{x}$ & $\mathrm{x}$ & $x$ & - & - & - & - & - & - & - & - & - & - & - & - & - & - & - & - & - & - & - & $\mathrm{x}$ \\
\hline 9 & $\mathrm{x}$ & $\mathrm{x}$ & $\mathrm{x}$ & $\mathrm{x}$ & $x$ & $\mathrm{x}$ & $\mathrm{x}$ & $\mathrm{x}$ & $\mathrm{x}$ & $\mathrm{x}$ & $\mathrm{x}$ & $\mathrm{x}$ & $\mathrm{x}$ & $\mathrm{x}$ & $\mathrm{x}$ & - & - & - & - & - & - & - & - & - & - & - & - & - & - & - & - & - \\
\hline 10 & $\mathrm{x}$ & $\mathrm{x}$ & $\mathrm{x}$ & $\mathrm{x}$ & $\mathrm{x}$ & $\mathrm{x}$ & $\mathrm{x}$ & $x$ & $\mathrm{x}$ & $\mathrm{x}$ & $\mathrm{x}$ & $\mathrm{x}$ & $\mathrm{x}$ & $\mathrm{x}$ & - & - & - & - & - & - & - & - & - & - & - & - & - & - & - & - & - & $\mathrm{x}$ \\
\hline 11 & $\mathrm{x}$ & $\mathrm{x}$ & $\mathrm{x}$ & $\mathrm{x}$ & $\mathrm{x}$ & $\mathrm{x}$ & $\mathrm{x}$ & $x$ & $\mathrm{x}$ & $x$ & $\mathrm{x}$ & $\mathrm{x}$ & $\mathrm{x}$ & $\mathrm{x}$ & $\mathrm{x}$ & $\mathrm{x}$ & - & - & - & - & - & - & - & - & - & - & - & - & - & - & - & - \\
\hline 12 & - & - & - & - & - & - & - & - & - & - & - & - & - & - & - & - & - & - & $\mathrm{x}$ & $\mathrm{x}$ & $\mathrm{x}$ & $\mathrm{x}$ & $\mathrm{x}$ & $\mathrm{x}$ & $\mathrm{x}$ & $\mathrm{x}$ & $\mathrm{x}$ & $\mathrm{x}$ & $\mathrm{x}$ & $\mathrm{x}$ & $x$ & $\mathrm{x}$ \\
\hline 13 & $\mathrm{x}$ & $\mathrm{x}$ & $\mathrm{x}$ & $\mathrm{x}$ & $\mathrm{x}$ & - & - & - & - & - & - & - & - & - & - & - & - & - & - & - & - & - & $\mathrm{x}$ & $\mathrm{x}$ & $\mathrm{x}$ & $\mathrm{x}$ & $\mathrm{x}$ & $\mathrm{x}$ & $\mathrm{x}$ & $\mathrm{x}$ & $\mathrm{x}$ & $\mathrm{x}$ \\
\hline 14 & $\mathrm{x}$ & $\mathrm{x}$ & $\mathrm{x}$ & $\mathrm{x}$ & $\mathrm{x}$ & $x$ & $\mathrm{x}$ & $x$ & $x$ & - & - & - & - & - & - & - & - & - & - & - & - & - & - & - & - & - & - & $\mathrm{x}$ & $x$ & $x$ & $x$ & $\mathrm{x}$ \\
\hline 15 & $\mathrm{x}$ & $\mathrm{x}$ & $\mathrm{x}$ & $\mathrm{x}$ & $\mathrm{x}$ & $\mathrm{x}$ & $\mathrm{x}$ & - & - & - & - & - & - & - & - & - & - & - & - & - & - & - & - & - & - & $\mathrm{x}$ & $\mathrm{x}$ & $\mathrm{x}$ & $\mathrm{x}$ & $\mathrm{x}$ & $\mathrm{x}$ & $\mathrm{x}$ \\
\hline 16 & $x$ & $\mathrm{x}$ & $\mathrm{x}$ & $\mathrm{x}$ & $\mathrm{x}$ & - & - & - & - & - & - & - & - & - & - & - & - & - & - & - & - & - & - & - & - & - & $\mathrm{x}$ & $\mathrm{x}$ & $\mathrm{x}$ & $\mathrm{x}$ & $\mathrm{x}$ & $\mathrm{x}$ \\
\hline 17 & $\mathrm{x}$ & $\mathrm{x}$ & $\mathrm{x}$ & $\mathrm{x}$ & $\mathrm{x}$ & $\mathrm{x}$ & $\mathrm{x}$ & $x$ & $\mathrm{x}$ & - & - & - & - & - & - & - & - & - & - & - & - & - & - & - & - & - & $\mathrm{x}$ & $\mathrm{x}$ & $\mathrm{x}$ & $\mathrm{x}$ & $\mathrm{x}$ & $\mathrm{x}$ \\
\hline 18 & $\mathrm{x}$ & $\mathrm{x}$ & $\mathrm{x}$ & $\mathrm{x}$ & $\mathrm{x}$ & $\mathrm{x}$ & $\mathrm{x}$ & $\mathrm{x}$ & $x$ & $\mathrm{x}$ & $\mathrm{x}$ & $\mathrm{x}$ & $\mathrm{x}$ & $\mathrm{x}$ & $\mathrm{x}$ & $\mathrm{x}$ & - & - & - & - & - & - & - & - & - & - & - & - & - & - & - & - \\
\hline 19 & $\mathrm{x}$ & $\mathrm{x}$ & $\mathrm{x}$ & $\mathrm{x}$ & $\mathrm{x}$ & $\mathrm{x}$ & $\mathrm{x}$ & $\mathrm{x}$ & $\mathrm{x}$ & $\mathrm{x}$ & - & - & - & - & - & - & - & - & - & - & - & - & - & - & - & - & - & - & $\mathrm{x}$ & $x$ & $x$ & $\mathrm{x}$ \\
\hline 20 & $\mathrm{x}$ & $\mathrm{x}$ & $\mathrm{x}$ & $\mathrm{x}$ & $\mathrm{x}$ & $\mathrm{x}$ & $\mathrm{x}$ & $x$ & $\mathrm{x}$ & $\mathrm{x}$ & $\mathrm{x}$ & $\mathrm{x}$ & $\mathrm{x}$ & $\mathrm{x}$ & $\mathrm{x}$ & - & - & - & - & - & - & - & - & - & - & - & - & - & - & - & - & - \\
\hline 21 & $\mathrm{x}$ & $\mathrm{x}$ & $\mathrm{x}$ & $\mathrm{x}$ & $\mathrm{x}$ & $\mathrm{x}$ & $\mathrm{x}$ & $\mathrm{x}$ & $\mathrm{x}$ & $\mathrm{x}$ & $\mathrm{x}$ & $\mathrm{x}$ & $\mathrm{x}$ & $\mathrm{x}$ & $\mathrm{x}$ & $\mathrm{x}$ & - & - & - & - & - & - & - & - & - & - & - & - & - & - & - & - \\
\hline 22 & $\mathrm{x}$ & $\mathrm{x}$ & $\mathrm{x}$ & $\mathrm{x}$ & $\mathrm{x}$ & $\mathrm{x}$ & $\mathrm{x}$ & $\mathrm{x}$ & $\mathrm{x}$ & $\mathrm{x}$ & - & - & - & - & - & - & - & - & - & - & - & - & - & - & - & - & - & $\mathrm{x}$ & $\mathrm{x}$ & $\mathrm{x}$ & $x$ & $\mathrm{x}$ \\
\hline 23 & $\mathrm{x}$ & $\mathrm{x}$ & $\mathrm{x}$ & $\mathrm{x}$ & $\mathrm{x}$ & - & - & - & - & - & - & - & - & - & - & - & - & - & - & - & - & - & - & - & - & - & $\mathrm{x}$ & $\mathrm{x}$ & $\mathrm{x}$ & $\mathrm{x}$ & $\mathrm{x}$ & $\mathrm{x}$ \\
\hline 24 & $\mathrm{x}$ & $\mathrm{x}$ & $\mathrm{x}$ & $\mathrm{x}$ & $\mathrm{x}$ & - & - & - & - & - & - & - & - & - & - & - & - & - & - & - & - & - & - & $\mathrm{x}$ & $\mathrm{x}$ & $x$ & $\mathrm{x}$ & $\mathrm{x}$ & $\mathrm{x}$ & $\mathrm{x}$ & $\mathrm{x}$ & $\mathrm{x}$ \\
\hline 25 & $\mathrm{x}$ & $\mathrm{x}$ & $\mathrm{x}$ & $\mathrm{x}$ & $x$ & $x$ & - & - & - & - & - & - & - & - & - & - & - & - & - & - & - & - & - & - & - & - & $\mathrm{x}$ & $\mathrm{x}$ & $\mathrm{x}$ & $\mathrm{x}$ & $x$ & $\mathrm{x}$ \\
\hline 26 & $\mathrm{x}$ & $\mathrm{x}$ & $\mathrm{x}$ & $\mathrm{x}$ & $\mathrm{x}$ & $\mathrm{x}$ & $\mathrm{x}$ & $\mathrm{x}$ & - & - & - & - & - & - & - & - & - & - & - & - & - & - & - & - & - & $x$ & $\mathrm{x}$ & $\mathrm{x}$ & $\mathrm{x}$ & $\mathrm{x}$ & $x$ & $\mathrm{x}$ \\
\hline 27 & $\mathrm{x}$ & $\mathrm{x}$ & $\mathrm{x}$ & $\mathrm{x}$ & $\mathrm{x}$ & $\mathrm{x}$ & $\mathrm{x}$ & $\mathrm{x}$ & $\mathrm{x}$ & $x$ & $\mathrm{x}$ & $\mathrm{x}$ & $\mathrm{x}$ & $\mathrm{x}$ & $\mathrm{x}$ & $\mathrm{x}$ & - & - & - & - & - & - & - & - & - & - & - & - & - & - & - & - \\
\hline 28 & $\mathrm{x}$ & $\mathrm{x}$ & $\mathrm{x}$ & $\mathrm{x}$ & $\mathrm{x}$ & $\mathrm{x}$ & $\mathrm{x}$ & $\mathrm{x}$ & $\mathrm{x}$ & $\mathrm{x}$ & $\mathrm{x}$ & $\mathrm{x}$ & $\mathrm{x}$ & $\mathrm{x}$ & $\mathrm{x}$ & - & - & - & - & - & - & - & - & - & - & - & - & - & - & - & - & - \\
\hline
\end{tabular}




\section{AppendixC. Summary of comparison studies}

We conducted the same comparison exercise presented in Section 8.2 to the two other case studies given in Section 7.1 and in Section 7.2, respectively. Table C.6 summarizes the comparison results for all the case studies. For the main case study presented in Section 6, we can establish the following conclusions: (i) the best final classification is $C_{4}$ followed by $C_{2}$ and than $C_{3}, C_{8}$ and $C_{6}$; (ii) the worst final classification is $C_{14}$ followed by $C_{16}$ and then $C_{10}$. In this particular case study, the final classifications obtained by a low value of the credibility threshold $\lambda$ are better than the ones obtained by high values of $\lambda$. Concerning the second case study, Table C.6 shows that the best classifications are $C_{1}$ and $C_{3}$ and the worst classifications are $C_{2}$ and $C_{4}$. According to the results in Table C.6, the final classifications obtained using the interval reduction rules 'max' or 'ceiling' $\left(C_{2}\right.$ and $\left.C_{4}\right)$ reproduce better the initial assignments than the final classifications obtained using the interval reduction rules 'min' or 'floor' $\left(C_{1}\right.$ and $C_{3}$ ). The results of the comparison study for the third case study shows that the best classification is $C_{10}$ (obtained for $\lambda=0.80$ and 'max' rule) and the worst classification is $C_{14}$ (obtained for $\lambda=0.95$ and 'max' rule).

Table C.6: Summary of comparison

\begin{tabular}{|l|cc|cc|cc|}
\hline & Case study 1 & \multicolumn{4}{c|}{ Tase study 3} \\
\hline Statistics & Worst classification & Best classification & Worst classification & Best classification & Worst classification & Best classification \\
\hline Kendall's $\tau$ & $C_{10}$ & $C_{4}$ & $C_{1}, C_{3}$ & $C_{2}, C_{4}$ & $C_{5}$ & $C_{10}$ \\
Spearman's $\rho$ & $C_{14}$ & $C_{4}$ & $C_{1}, C_{3}$ & $C_{2}, C_{4}$ & $C_{5}$ & $C_{10}$ \\
Cohen's $\kappa$ & $C_{14}$ & $C_{3}$ & $C_{2}, C_{4}$ & $C_{1}, C_{3}$ & $C_{14}$ & $C_{12}$ \\
\hline Kendall's $\tau+$ Spearman's $\rho$ +Cohen's $\kappa$ & $C_{14}$ & $C_{4}$ & $C_{1}, C_{3}$ & $C_{2}, C_{4}$ & $C_{14}$ & $C_{12}$ \\
\hline Kendall's $W$ & $C_{14}$ & $C_{4}, C_{8}$ & $C_{1}, C_{3}$ & $C_{2}, C_{4}$ & $C_{5}$ & $C_{10}$ \\
Fleiss's $\kappa$ & $C_{16}$ & $C_{2}$ & $C_{1}, C_{3}$ & $C_{2}, C_{4}$ & $C_{14}$ & $C_{1}$ \\
\hline Kendall's $W$ + Fleiss's $\kappa$ & $C_{14}$ & $C_{2}, C_{6}$ & $C_{1}, C_{3}$ & $C_{2}, C_{4}$ & $C_{14}$ \\
\hline
\end{tabular}

